
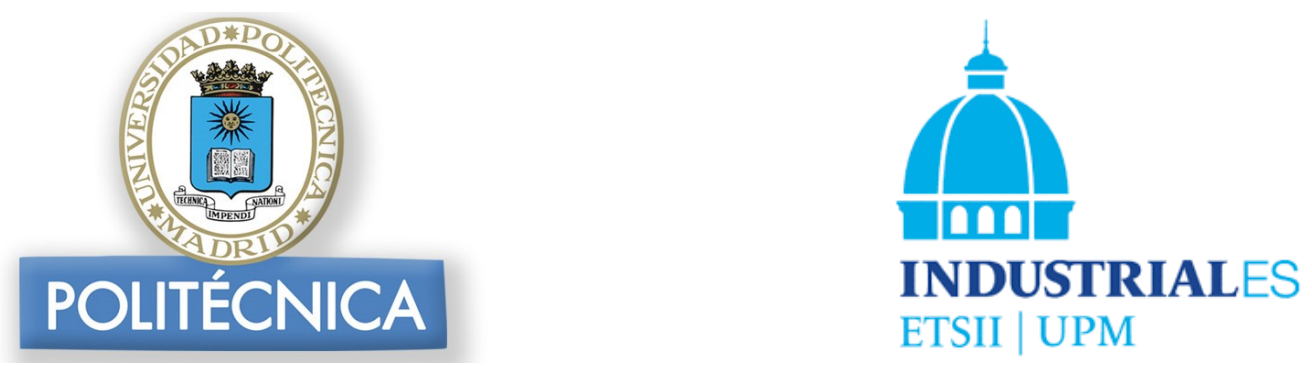

Universidad Politécnica de Madrid

Escuela Técnica Superior de Ingenieros Industriales

\title{
Uncertainty Quantification and Global Sensitivity Analysis in Computational Mechanics
}

Doctoral Thesis

Edoardo Menga

Ingeniero Industrial

Director: Prof. Ignacio Romero Olleros

Co-director: Prof. María J. Sánchez Naranjo

Madrid, 2020 

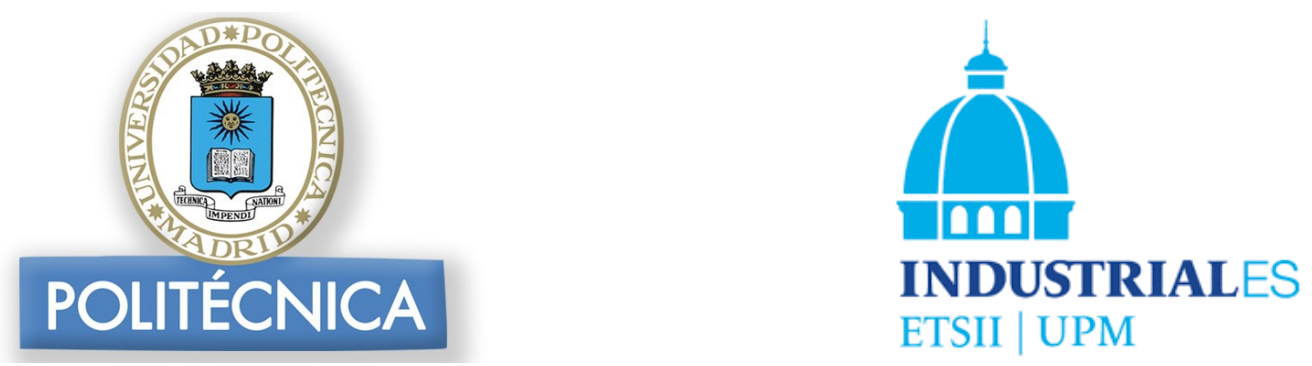

Universidad Politécnica de Madrid

Escuela Técnica Superior de Ingenieros Industriales

\title{
Uncertainty Quantification and Global Sensitivity Analysis in Computational Mechanics
}

Doctoral Thesis

\author{
Edoardo Menga \\ Ingeniero Industrial \\ Director: Prof. Ignacio Romero \\ Co-director: Prof. María J. Sánchez
}

Madrid, 2020 
Tribunal nombrado por el Magfco. y Excmo. Sr. Rector de la Universidad Politécnica de Madrid, el día . . . . . . . de . . . . . . . . de 2020.

Presidente: D. . . . . . . . . . . . . . . . .

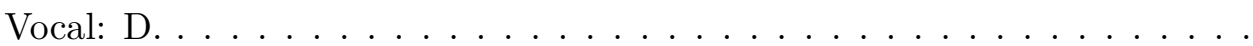

Vocal: D.

Vocal: D.

Secretario: D.

Realizado el acto de defensa y lectura de la tesis el día . . . . . . de . . . . . . . . de 2020, en la Escuela Técnica Superior de Ingenieros Industriales, de la Universidad Politécnica de Madrid

Calificación: 

A mio padre Elio

e a mio fratello Massimiliano 


\section{Ringraziamenti}

Quando penso a come é cominciata questa avventura del dottorato, non posso evitare di sentire ancora l'emozione dell'ammissione e, allo stesso tempo, la solitudine che ho provato quando, camminando per i corridoi di un'universitá a me sconosciuta, mi chiedevo chi avrebbe accettato, con i miei 38 anni, di essere il mio direttore di tesi.

Cosí, quasi casualmente, finí nello studio di Ignacio e, vedendo sulla sua scrivania vari fogli con gradienti ed equazioni differenziali, dubitai di quanto veramente mi sarei potuto compromettere in un progetto del genere. Ricordo, oggi quasi sorridendo, che furono giorni di grande incertezza!

Credo che Ignacio decise di darmi una possibilitá e mi propose, quasi come se sapesse quello che stessi provando, di addentrarmi insieme a lui nel mondo della quantificazione dell'incertezza. Un campo di studio completamnete nuovo anche per lui. Certamente non sarebbero mancati elementi e principi di statistica, cosicché reclutammo la mia condirettrice: Mariaje. Cosí cominciarono "i nostri Venerdí”!

Ringrazio entrambi per il tempo e l'attenzione che mi hanno dedicato. É stato un percorso pieno, ricco di momenti di incessanti lavoro e soddisfazione, che mi hanno permesso di crescere professionalmente e personalmente. La tranquillitá, l'ottimismo e l'integritá professionale di Ignacio, cosí come la passione e la forza di vita di Mariaje, giunto quasi alla fine di questo percorso, li sento anche un pó miei. Con rigore accademico, entrambi, hanno saputo trasmettermi fiducia e motivazione per poter completare, al meglio delle mie possibilitá, i miei studi di dottorato. Grazie di cuore!

Ringrazio, inoltre, Consuelo Huerta della disponibilitá. Il suo aiuto é stato fondamentale per completare la campagna sperimentale nel laboratorio di prove meccaniche del dipartimento di ingegneria meccanica.

Allo stesso modo non posso dimenticare i ringraziamenti a Santiago per la relazione professionale e personale che, in svariati anni di collaborazione, attraverso AIRBUS, abbiamo saputo costruire. Momenti di pressione e duro lavoro sempre 
ricompensati dal conseguimento di ottimi risultati. Qualche anno fa, insieme, siamo riusciti a sviluppare un metodo per la dinamica strutturale non lineare che ha ispirato un capitolo di questa tesi.

Non posso nascondere che il cammino é stato lungo.

Quando cominciai, mia figlia, Nora, era una bimba che potevo ancora portare in braccio, oggi é giá un' adolescente. Mio figlio, Elía, arrivato nel corso di questi ultimi anni , ci ha regalato una nuova energia e, tra meno di due mesi, compirá tre anni.

Ringrazio la mia compagna di vita, Vicky, che allevia sempre i momenti di difficoltá con i suoi deliziosi piatti di cucina messicana. Spesso é stata in grado di regalarmi la tranquillitá di cui avevo bisogno per concentrarmi negli studi.

Sono loro a dare un senso alla mia "ricerca della felicitá" e che, ogni giorno, mi motivano a costruire, insieme, una vita piena.

I miei genitori, Elio e Lia, sono la vera ragione per cui oggi posso completare questo lavoro di tesi. Mi hanno sempre dato forza, amore e fiducia incondizionati.

Mi hanno insegnato a vivere la vita con coraggio e rispetto. Perfino in un momento molto difficile, hanno accettato e rispettato la scelta che feci di andarmene dall' Italia. Mi hanno sempre lasciato libero di cercare la mia propria strada. Mi hanno insegnato a non eludere la responsabilitá, a gioire per le cose semplici e vere, ad alzarmi con rinnovata forza tutte le volte che sia necessario. "Gutta cavat lapidem non vi, sed saepe cadendo", spesso mi diceva mio padre.

Un pensiero speciale di tenerezza e amore infiniti va a mio fratello Massimiliano. Indelebili le nostre risate, il suo sorriso e la sua forza vivono in me. Un forte abbraccio a mia sorella, Danila che mi ha aiutato a scrivere questi ringraziamenti in un italiano non spagnolizzato! 


\section{Agradecimientos}

Cuando pienso en como empezó ese trabajo de doctorado aún siento la emoción de cuando me admitieron, pero a la vez también la soledad que probé cuando caminando por lo pasillos de una universidad desconocida, me preguntaba quien podría aceptar, a mis 38 años, ser mi director de tesis. Casi por casualidad, acabé en el despacho de Ignacio, y viendo sus hojas con gradientes y ecuaciones diferenciales, dudé profundamente si hubiera podido comprometerme a "algo así". ¡Fueron días de gran incertidumbre!

Creo que Ignacio quiso darme una posibilidad y me propuso, casi sabiendo lo que yo estaba sintiendo, de adentrarnos en el mundo de la cuantificación de incertidumbre. Algo nuevo también para él. Y como "algo de estadística" se veía venir, buscamos el apoyo de mi co-directora: Mariaje. ¡Así empezaron "nuestros Viernes"!

Sinceramente agradezco a ambos por el tiempo y las atenciones que me han dedicado. Ha sido un recorrido muy enriquecedor, lleno de momentos de incesante trabajo y satisfacción, que me ha permitido crecer profesionalmente y personalmente. La tranquilidad, el optimismo y la integridad profesional de Ignacio, así como la pasión y la fuerza de vida de Mariaje, ahora también hacen un poco parte de mí. Con rigurosidad académica, ambos han sabido trasmitirme confianza y motivación para completar, como mejor he sabido, mis estudios de doctorado. ¡Gracias!

Agradezco a Consuelo Huerta su disponibilidad. Gracias a su ayuda pude completar la campaña de ensayos en el laboratorio del departamento de ingeniería mecánica.

No puedo dejar de agradecer a Santiago, la relación profesional y personal que en varios años de colaboración, a través de AIRBUS, hemos sabido construir. Los momentos de presión y duro trabajo siempre han sido gratificados por los excelentes resultados conseguidos. Hace unos años, juntos pudimos desarrollar un método de dinámica no-lineal que ha sido de inspiración para una parte de esta tesis. 
Ha sido un recorrido largo.

Cuando empecé mi hija Nora era una niña que aún podía cargar, hoy es una adolescente. Mi hijo Elía ha llegado en el medio del camino para regalarnos nueva energía e en menos de dos meses, va a cumplir tres años. Agradezco a mi compañera de vida Vicky por aliviar los momentos mas duros con su deliciosa cocina mexicana. Muchas veces ha sabido regalarme la tranquilidad para poder concentrarme en mis estudios. Ellos dan un sentido a mi "búsqueda de la felicidad" y me motivan a que juntos podamos construir una vida plena.

Mis padres, Elio y Lia, son la verdadera razón por la cual yo hoy soy capaz de terminar este trabajo de tesis. Siempre, incondicionalmente, me han trasmitido fuerza, amor y confianza. Aún en un momento difícil de nuestra vida, han aceptado y respetado mi elección de marcharme de Italia. Siempre me han dejado libre de encontrar mi propio camino. Ellos me han enseñado a no evadir la responsabilidad, a disfrutar las cosas sencillas y verdaderas de la vida, y a levantarme con renovada energía todas las veces que haga falta. "Gutta cavat lapidem non vi, sed saepe cadendo", me solía decir mi padre.

Un pensamiento especial, con profunda ternura y amor, va a mi hermano Massimiliano. Indeleblemente, nuestras risas, su sonrisa y su fuerza viven en mí. Un fuerte abrazo a mi hermana, Danila.

Gracias. 



\section{Contents}

1 Introduction $\quad 23$

1.1 Premise and motivation . . . . . . . . . . . . . . 23

1.2 Sample-based uncertainty quantification process . . . . . . . . . 25

1.2 .1 Pre-processing . . . . . . . . . . . . . . 26

1.2.2 Uncertainties Propagation . . . . . . . . . . . . . 28

1.2 .3 Post-processing . . . . . . . . . . . . . . . . . . 30

1.3 Motivations and thesis' objectives . . . . . . . . . . . . 33

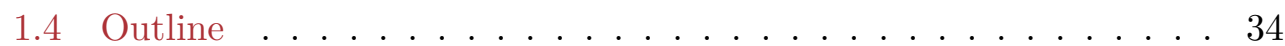

2 A perspective on Sobol's sensitivities based on an experimental $\begin{array}{ll}\text { campaign } & \mathbf{3 7}\end{array}$

2.1 Introduction . . . . . . . . . . . . . . . . . . . 37

2.2 High Dimensional Model Representation . . . . . . . . . . . . . . . 38

2.2.1 Variance decomposition and sensitivity indices . . . . . . 39

2.2.2 Estimation of sensitivity indices . . . . . . . . . . . . 40

2.3 Experimental set-up . . . . . . . . . . . . . . . . . . 42

2.4 Factorial Design and Sensitivities . . . . . . . . . . . . . . 45

2.4.1 Analytical evaluation of Sobol's indices . . . . . . . . 47

2.4.2 Estimation of Sobol's indices by the coefficient of determination in regression . . . . . . . . . . . . 51

2.4.3 Estimation of Sobol's indices by Low Discrepancy Design . 53

2.5 Conclusions . . . . . . . . . . . . . . . . . . . 54

3 Meta-models $\quad 57$

3.1 Introduction . . . . . . . . . . . . . . . . . . 57

3.1.1 Outline of the chapter . . . . . . . . . . . 57

3.2 Scatter data approximation by the least square method . . . . . . 58

3.2.1 Meta-model performance: efficiency and predictiveness . . . 60

3.2.2 Considerations on meta-model assessment and selection . . 62 
3.3 Meta-models based on radial kernels . . . . . . . . . . . . . . 63

3.3.1 Shape parameter and anisotropic metrics . . . . . . . . 64

3.3.2 Sobol LDD, GSA and anisotropic metrics . . . . . . . . 66

3.3 .3 Detailed methodology . . . . . . . . . . . . . 67

3.4 Applications . . . . . . . . . . . . . . . . . 68

3.4.1 Sobol's G-function . . . . . . . . . . . . . . 69

3.4.2 Piston simulation function . . . . . . . . . . . . 71

3.4 .3 Taylor's impact test . . . . . . . . . . . . . 78

3.5 Conclusions and future work . . . . . . . . . . 87

4 Reduction Order Methods and Meta-models $\quad 90$

4.1 Introduction . . . . . . . . . . . . . . . . . . 90

4.1 .1 Outline of the chapter . . . . . . . . . . . . . . 91

4.2 Nonlinear Structural Dynamic Modifications . . . . . . . . . . . . . 92

4.3 Uncertainty Quantification . . . . . . . . . . . . . . . . . . 95

4.3.1 Meta-model: Polynomial Chaos Expansion . . . . . . . 95

4.3.2 Global Sensitivity Analysis . . . . . . . . . . . . . . 98

4.3.3 Polynomial Chaos and Sobol's Indices . . . . . . . . . . 98

4.4 A380 RAM Air Inlet free-play nonlinear vibrations . . . . . . . . . 100

4.4.1 Computational approach . . . . . . . . . . . . . . 103

4.4 .2 Results . . . . . . . . . . . . . . . 108

4.5 Conclusions and future work . . . . . . . . . . . . . 110

5 Summary and conclusions $\quad 111$

5.1 Main results of the thesis . . . . . . . . . . . . . 113

5.2 Future work . . . . . . . . . . . . . . . . . . . . . . 114

5.3 Scientific publications and conference presentations . . . . . . . 115

$\begin{array}{ll}\text { A Optimal regularization for ill-conditioning } & 117\end{array}$

Bibliography 122 


\section{List of Figures}

1.1 Uncertainty Quantification overview. . . . . . . . . . . . . . 26

2.1 An example of construction of $A B_{i}$ matrices with $N_{p}=3$ and $N_{s}=4 .($ source $\mid$ Wikipedia $) \ldots \ldots \ldots \ldots 4$

2.2 Beam test set-up at mechanical laboratory of the Mechanical Engineering department of the UPM. Three accelerometers are installed, two at the beam's ends and one in the centre in order to characterize the dynamic behaviour. . . . . . . . . . . . 42

2.3 Beam section details. . . . . . . . . . . . . . . . . . . . . 42

2.4 Beam's first three elastic modes. . . . . . . . . . . . . . . . . 44

2.5 2-level 3-variables FFD. . . . . . . . . . . . . . . . . . 45

2.6 Sobol's main effects for first three frequencies. . . . . . . . . . . . . 51

2.7 Comparison between the main effects for the first three frequencies. The first group (blue bars) takes in consideration the $S_{i}$ calculated analytically by close-form formula. The second group (orange bars) takes in consideration the $S_{i}$ estimated by the $R^{2}$ regression approach. The differences are negligible. . . . . . . . 53

2.8 Convergence of Sobol's main effects vs. sample size. The picture shows the case of the third output frequency. Because of Saltelli approach is based on Sobol' LDD re-sampling matrices, an high number of samples is required to achieve the convergence and consequently a good estimation of the indices. . . . . . . . . . . . 54

2.9 Convergence of Sobol's total effects vs. sample size. The picture shows the case of the third output frequency. Because of Saltelli approach is based on MC re-sampling matrices, an high number of samples is required to achieve the convergence and consequently a good estimation of the indices. . . . . . . . . . . . . 
2.10 Comparison between the main effects for the first three frequencies. The first group (blue bars) takes in consideration the $S_{i}$ calculated analytically. The second group (orange bars) takes in consideration the $S_{i}$ estimated by LDD. The differences are negligible but the first are estimated by using directly the 8 test results, while LDD requires a much higher number of results, even in case of categorical variables. In this case $N_{s}=2048$ sample points are used. . . . . . .

3.1 Meta-model construction: a sample matrix is considered and the QoIs evaluated through the simulation model. Then, this set is split in a usually wider training set where the coefficients of regression are estimated and in a smaller evaluation set, the unseen set of data, where the error can be evaluated. . . . . . . . . . .

3.2 Meta-model approximation: depending on the number of basis functions $N_{k}$ in relation to the number of simulations points $N_{s}$, the least square solution can be an interpolation $\left(N_{k}=N_{s}\right)$ or an approximation $\left(N_{k}<N_{s}\right) \ldots \ldots \ldots \ldots \ldots$

3.3 Anisotropic kernel matrix: computationally, the generic anisotropic kernel $K_{s, k}$ can be obtained multiplying each column of the sample by the corresponding anisotropic metric $\gamma_{p} \ldots \ldots \ldots \ldots$

$3.4 G$-function in dimension 15 - error vs. shape parameter. The plot compares the solution obtained through the ordinary LSM (continuous lines) with those obtained with anisotropic metrics (discontinuous lines) with different sizes of the training set. In general, anisotropic metrics provide smaller errors that, furthermore, are almost independent of the shape parameter. . . . . . . . . . . 71

3.5 Piston function - GSA indices. . . . . . . . . . . 77

3.6 Schematic of anvil in Taylor's impact test. . . . . . . . . . . . 78

3.7 Taylor's test: anvil's diameter increment. Errors obtained by using radial kernels (continuous lines) and by enforcing anisotropic metric (discontinuous lines) vs. decreasing value of the shape parameter $\epsilon$. Four sizes are considered for the training set: 32, 64, 128,256 points. In the case of enforced anisotropy, the error is less sensitive to the size of the training set and almost independent from the shape parameter. . . . . . . . . . . . . . 
3.8 Errors obtained by using radial kernels with optimised anisotropic metrics vs. wider training set. For each set three values of the shape parameter are considered, i.e, 0.5 (blue), 0.3(orange) and 0.1 (grey) bars. The value assigned to the shape parameter does not have a relevant effect on the error when the metrics are optimised. 82

3.9 Diameter increment - sensitivity indices results: according to the prescribed distribution only two outputs, the velocity and the material parameter $B$, have influence on the output variance of the diameter increment. All the rest can be fixed at its nominal value.

3.10 Anvil's length reduction - sensitivity indices results: for this QoI the velocity and the material parameter $B$ influence the output's variance the most. . . . . . . . . . . . . . . . 84

3.11 Probability density function of the diameter increment after the impact. The distribution is Gaussian with a mean $\mu=3.304$ and a standard deviation $\sigma=0.175 \ldots \ldots \ldots \ldots \ldots$. . . . . .

3.12 Probability density function of the length reduction after the impact. The distribution is Gaussian with a mean $\mu=8.114$ and a standard deviation $\sigma=0.343 \ldots \ldots \ldots \ldots \ldots$

3.13 The trend of the length reduction vs. velocity is multi-lineal. A little interaction of velocity with the other parameters arises because the slope of the curves is not exactly the same when the set of input parameters is changed. Given a set of parameters by using the multi-lineal curves, one can obtain the length reduction (and equivalently the diameter increment) at each velocity by a simply linear interpolation. . . . . . . . . . . . .

3.14 Figure shows the comparison between the results obtained by simulations and those obtained by employing the meta-model interpolation approach. Simulation points are plotted in blue, while interpolated meta-models results are in orange. . . . . . . . . .

3.15 Histogram of the error over the 500 simulations. The mean error is $0.006 \ldots \ldots \ldots \ldots \ldots$

4.1 Multi-linear force-displacement curve. . . . . . . . . . . . . . . . 93

4.2 A380 - RAI System and Air Intake detail (source|AIRBUS OPERATIONS) . . . . . . . . . . . . . . . . 101

4.3 A380 - RAI System Flaps (source|AIRBUS OPERATIONS). . . . 102

4.4 Kinematic scheme of flaps control system (source|AIRBUS OPERATIONS) . . . . . . . . . . . . . . . . 102 
4.5 3D design of the flaps control system (source|AIRBUS OPERATIONS) . . . . . . . . . . . . . . . . . 103

4.6 RAI FE Model . . . . . . . . . . . . . . . . . . . . . . . . . . . . 104

4.7 SDMM updating method vs. NASTRAN: in this Figure both time and frequency domain response of the system are compared when the springs at interfaces between the rods and the arms are desconnected (red line) and connected (blue line). Both solutions are obtained in NASTRAN. Additionally, the response with connected springs is obtained through updating the disconnected response by means of the SDMM. The obtained response (orange line) overlap the NASTRAN's solution. Hence, the SDMM is able to consider disruptive change of local stiffnesses in both domains. . . . . . . . 105

4.8 Nonlinear stiffness and reaction force at nominal conditions as defined in table 4.5 . . . . . . . . . . . . . . . 106

4.9 Comparison between linear and nonlinear rod's reaction force at nominal conditions as defined in table 4.5 . . . . . . . . . 107

4.10 Nonlinear interfaces behavior: uncertain free-play stiffness. . . . 107

4.11 Reaction force $F 1_{r m s}$ - Pie charts of first and total indices. The output's variance cannot be explained completely by the first order effects because interactions among inputs are relevant. Particularly, seeing at the total effects, the stiffness $K_{1}$ and $K_{2}$ acquire more relevance. In any case the uncertainty of the response is dominated by the frequency of excitation first, and then by the magnitude of the applied force and by the size of the gaps of freeplays. . . . . . . . . . . . . . . . . . . 109 


\section{List of Tables}

2.1 Beam's properties. . . . . . . . . . . . . . . . . . 43

2.2 Masses of the accelerometers installed on the beam. . . . . . . . . 43

2.3 Comparison between experimental and numerical results. . . . . . 43

2.4 Dead-masses properties and prescribed probabilities. . . . . . . . . 45

2.5 2-level 3-variables FFD and frequency results. . . . . . . . . . . 46

2.6 Normalized values of frequencies in 2-level FFD. . . . . . . . . . 47

2.7 Regression coefficients in 2-level FFD. . . . . . . . . . . . . 47

2.8 Prescribed probabilities on dead-masses existence. . . . . . . . . . 48

2.9 Decomposition of the total variance for the three masses problem. In this exercise $\sigma^{2}\left(X_{i}\right)=p(1-p)=0.25$, for $i=1,2,3$ and $p=0.5$. The term $\sigma^{2}\left(X_{i} \cdot X_{j}\right)=p^{2}\left(1-p^{2}\right)=0.1875$, for $i, j=1,2,3$ with $i<j$. These formulas are still valid for a different value of $p$ but one has to consider that by changing $p$, the output distribution changes accordingly and then the $\beta$ coefficients need to be re-calculated by a new regression. . . . . . . . . . . . . . . . 50 50

2.10 Sobol's sensitivities obtained by recursive analytical formulas. . . . 51

2.11 Sobol's sensitivities obtained by recursive regression. . . . . . . . . 52

2.12 Sobol's sensitivities obtained by Saltelli approach. . . . . . . . . . 54

$3.1 G$-function in dimension 15 - First order sensitivity indices: comparison between analytical results and those obtained numerically by a meta-model with anisotropic kernel. The surrogate is constructed by employing a training set of $N_{t r}=1024$ points and a shape parameter $\varepsilon=3 \ldots \ldots \ldots \ldots \ldots \ldots 72$

3.2 Piston function - random variables. . . . . . . . . . . . . . 73

3.3 Piston function - anisotropic metrics from [32, chapter 16] . . . . . 73

3.4 Piston function - Halton vs. Sobol' LDD. . . . . . . . . . . . . . 75

3.5 Piston function - error evaluation with optimised anisotropic metric. 75 
3.6 Piston function - optimised anisotropic metric. . . . . . . . . 76

3.7 Piston function - First order and total sensitivity indices. . . . . . 77

3.8 Johnson-Cook constants for copper. . . . . . . . . . . . . . 79

3.9 Anvil's diameter increment. For training sets of four different sizes, blocks report the errors obtained by considering only the shape parameter $\epsilon$ with or without optimised anisotropic metrics. In the third line of each block of results, the relative improvement on the error is shown. . . . . . . . . . . . . . . 81

3.10 Experimental results presented in [75] for the impact tests of a copper cylinder ( $L_{i}$ : initial length of the anvil; $L_{f}$ : final length). When the impact velocity is $190 \mathrm{~m} / \mathrm{s}$, the experimentally measured length reduction is very close to the mean value of the Gaussian of Figure 3.12 (i.e. $\mu=8.114 \mathrm{~mm}$ ) . . . . . . . . . . . . .

3.11 JC model - length decrement: 5 surrogates are generated to evaluate the Sobol's Indices at 5 different impact velocity. Mean, variance and sensitivities, main and total effects, are provided in the table. Results confirm that the relative relevance of the material's parameters is slightly sensitive to the change in velocity. Additionally, the main effect cover already almost the $100 \%$ of the total variance, hence no relevant interactions among material's parameters exist. Finally, the parameters $\mathrm{N}$ and $\mathrm{M}$ can be fixed at their nominal values. . . . . . . . . . . . . . . . 88

4.1 Correspondence between orthogonal polynomials and PDF. . . . . 96

4.2 Bivariate second order Hermite polynomials. . . . . . . . . . . 97

4.3 Multi-index: bivariate PCE of $2^{\text {nd }}$ order. . . . . . . . . . . . 99

4.4 Equivalence between Sobol's HDMR and PCE. . . . . . . . . . . 100

4.5 Statistical distributions of the random variables. . . . . . . . . 106

4.6 RAI vibrations - statistical moments and Sobol's Indices. . . . . . 109 


\section{Acronyms}

AIC Akaike's Information Criterion. 61

BIC Schwarz Bayesian Information Criterion. 61

DM Distance Matrix. 61, 62, 64

FAST Fourier Amplitude Sensitivity Test. 30

FE Finite Element. 89, 90, 92, 111

FFD Full Factorial Design. 35, 43, 44, 52, 109

GCV Generalized Cross-Validation. 61, 62, 116

GSA Global Sensitivity Analysis. 21-23, 27-33, 56, 58, 72-75, 84-86, 88, 90, 96, 101, 108-113

HDMR High Dimensional Model Representation. 27, 29, 32, 35, 36, 96-98, 108, 110

KS Kolmogorov-Smirnov. 30, 31

LDD Low Discrepancy Design. 26, 39, 40, 51, 65, 72, 86, 93

LHD Latin Hypercube Design. 26, 93

LOOCV Leave-One-Out Cross Validation. 60, 61

LSM Least Square Method. 55, 65, 68

MC Montecarlo. 26, 39, 93

NL SDMM Nonlinear Structural Dynamic Modifications Method. 33, 89, 90, 108,110 
OAT One-factor-At-Time. 43

PCE Polynomial Chaos Expansion. 27, 33, 34, 89, 90, 93, 95-98, 108, 110

POD Proper Orthogonal Decomposition. 27

QoIs Quantities of Interest. 21, 22, 25, 26, 28-31, 43, 65, 66, 77, 80, 81, 88, 93, 94, 96, 104, 106, 108

R2 coefficient of determination in regression. 35, 50

RBF Radial Basis Function. 27, 61, 62, 108

RK Radial Kernel. 27

ROM Reduced Order Method. 30, 33, 110

SDMM Structural Dynamic Modifications Method. 89, 108

SVD Singular Value Decomposition. 116, 118

UP Uncertainty Propagation. 26, 28-30, 96

UPM Universidad Politécnica de Madrid. 40, 109

UQ Uncertainty Quantification. 21-23, 25, 27, 30-33, 51, 56, 58, 66, 76, 77, 84, 88, 89, 93, 98, 108-110, 112 


\section{Abstract}

This thesis provides insight on Uncertainty Quantification (UQ) and Global Sensitivity Analysis (GSA) processes and explores in detail those aspects of metamodels which make them suitable for real applications. For this purpose, UQ, GSA, and the construction of well-designed meta-models, instrumental to complete both processes, are revisited by providing a balance between a rigorous mathematical approach and practical guidelines needed in an industrial environment.

This work is motivated by the advantages that derive, at the industrial level, from the adoption of an effective UQ/GSA framework: a one-time process of product development with increases reliability and durability and the opportunity to provide more credible and realistic simulations. The required additional efforts, essentially an investment in people skills and some extra time in the design and engineering phases of the project, have a clear return of investment in the midand long-term. For the reasons outlined, large companies such as AIRBUS are investing large amounts of funds in the development of rigorous, yet practical, UQ/GSA frameworks that are tightly coupled with their simulation workflows.

In this thesis, a clear description and an exhaustive literature review on UQ and GSA processes are provided. A new perspective is provided for Sobol's sensitivity indices and Saltelli's resampling approach, the technique usually employed to estimate them. This is done first in light of an experimental campaign. Also, a novel link with the coefficients of approximation in regression is identified.

Building well-design metamodels is instrumental to complete UQ and GSA processes. Because of that, a rigorous framework for metamodels evaluation and selection is provided and a novel methodology to systematically construct quasioptimal metamodels is proposed. Specifically, a link between anisotropic metrics and Sobol's sensitivities is explored and quantified using GSA. As an example of the usefulness of well-designed metamodels, some analytical functions and two industrial applications are studied. In particular, the sensitivities are calculated for the material model of Johnson and Cook, illustrating, for the first time, the 
use of UQ/GSA to guide the parameter fitting of complex constitutive models. The ideas are, however, general and can be used to steer the formulation of effective meta-models in other complex experimental setups.

Finally, an application to a real aeronautical structure is considered. The novelty of this thesis is in the incorporation in the UQ process of a nonlinear Order Reduction Method (ROM). Combining ROM with meta-models is shown to be a suitable and pragmatic approach proving that the methodology presented in this thesis can be extended to computational models where the uncertainties can be localized at lumped parameters. 


\section{Chapter 1}

\section{Introduction}

\subsection{Premise and motivation}

The American mathematician John Allen Paulos said: "Uncertainty is the only certainty there is, and knowing how to live with insecurity is the only security". The budding field of Uncertainty Quantification (UQ) is the product of this philosophy and those like it. According to definitions from the National Agency for Finite Element Methods and Standards (NAFEMS) Stochastics Working Group and the American Society of Mechanical Engineers (ASME) Standards Committee on Verification and Validation, uncertainty quantification is the formulation of a statistical model to characterize imperfect and/or unknown information in engineering simulation and physical testing for predictions and decision-making. UQ seeks to address the problems associated with incorporating real-world variability and probabilistic behaviours into engineering and systems analysis.

In a more formal way Uncertainty Quantification can be defined as the science of identifying, quantifying and reducing uncertainties associated with the models, numerical implementations, experiments and quantities of interest [1].

UQ takes its bricks from classical statistics and numerical analysis theory, and includes them in a more systematic approach which takes into account the uncertainties of the inputs, how they are propagated through the model, which the output's density distributions and their statistical moments are, and, in a global sense, how sensitive the outputs are with respect to the prescribed uncertain inputs. The study of these sensitivities between a set of stochastic inputs and a set of Quantities of Interest (QoIs), i.e. those outputs which provide information necessary to make conclusions or decisions about the process [1], is accomplished by the Global Sensitivity Analysis (GSA). 
In this research the concept of UQ and GSA are explored in the frame of reliability and predictiveness of numerical models in computational mechanics. In fact, many companies in the field of mechanics, aeronautics, energy, etc. rely on complex, high-fidelity simulations to virtual-test their products, to predict in-service issues, to offer a better support in case of design modifications, and more and more often for optimisation. In other words, engineering design is concerned with making decisions based on analysis, which directly impact the product or service being designed and numerical models (i.e. Finite Element models) are expected to provide analysts with a robust engineering framework for that purpose. A computational model can be from an analytical function in the simplest case to a combination of pieces of software and physical experiments in more complex cases. In the end, the model provides a map between a set of inputs and defined QoIs [2]. It is considered to be deterministic, i.e., the repeated evaluation with the same set of input parameters leads to the same model response.

A first link between UQ and numerical models is the concept of non-intrusive or sample-based approach. It uses the inputs and responses from multiple deterministic simulations to build a predictive statistical model. Then, UQ is a probabilistic approach that can be described as a deterministic approach that is systematically repeated according to some specific sampling design. Research activities exist in the field of optimisation and robust design to improve the feasibility of sample-based, or non-intrusive, UQ and GSA [3, 4, 5].

A key concept in UQ is the use of meta-models. Despite the continuous increase in computing power, the computational cost required for one deterministic simulation can still so high that it is difficult, often prohibitive, to explore satisfactorily the design space of the product, particularly if many input variables are involved in the process. Hence, although non-intrusive methods are now established in the engineering community as a pragmatic approach to both UQ and GSA especially for industrial applications, there exists a need to replace any complex model with an approximation, called meta-model or surrogate, whose solution requires less computational cost, but having a similar response. In this sense meta-models are viewed as defining a relationship between a set of inputs and a set of outputs, valid within a certain domain of the inputs and for a set of predefined QoIs.

Implementing UQ at the industrial level has many benefits, the most important ones being:

A one-time process for product development: UQ enables the ability to 
have a one-time process for product development. Variability can be viewed as a cost driver when designing or analyzing a new model or system. The probabilistic approach used with UQ reduces these process iterations that can happen during the design or analysis phases. Additional cost reductions are realized when each design iteration includes procuring and testing prototypes.

Optimization of product reliability and durability: this can be accomplished in the design phase by conducting GSA to first identify which variables have the greatest influence on the output variation. The next step would be considering the relevant variables to find an optimum that improves the reliability and durability performance. Understanding the robustness of the optimum can be assessed by propagating uncertainties through the model.

Ensure simulation results are credible and realistic: when a model is used with a deterministic approach, often security factors and sometimes extreme values of load and material strength are used instead of expected values. Thus, the philosophy behind a model reflects this worst conditions approach because if the results are favorable in meeting target lifetime goals, then it will likely survive under the expected conditions. However, that is not necessarily credible nor realistic, and affects how trustworthy the model can be when using it to make predictions. The UQ process, which maps the input-output relationship, generates a trustworthy (e.g., model validation) and that is essential for understanding the risks when using simulation results in the decision-making process.

Government oversight: The benefits of modeling and simulation have been recognized at a policy level by several federal agencies (such as the EASA, EDA, FAA and DOD) of the European and U.S. government. Agencies are evaluating methods to verify and validate computer models such that the simulation results are trustworthy sources of information capable of meeting stringent federal regulations. The basis of validation methods is UQ.

In the next section the fundamental bricks of the UQ process, in conjunction with GSA, are reviewed in detail and discussed along with relevant bibliographic references.

\subsection{Sample-based uncertainty quantification process}

UQ can be thought as a process with three fundamental phases, namely, pre-processing, uncertainties propagation, and post-processing, each of them including different tasks. Figure 1.1 shows schematically these three stages and their breakdown, as well as the data-flow within the process. 


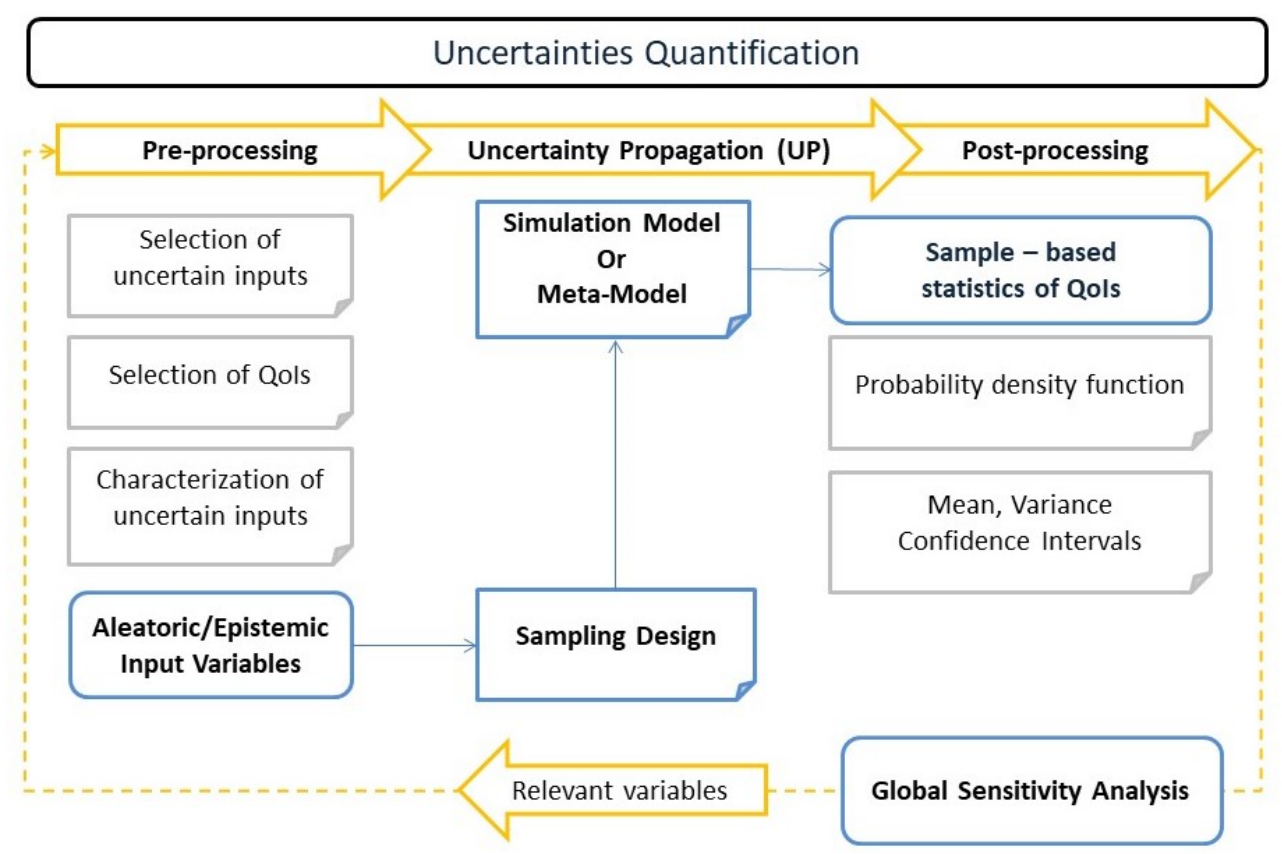

Figure 1.1: Uncertainty Quantification overview.

\subsubsection{Pre-processing}

As the name suggests, pre-processing refers to all tasks that need to be done in order to prepare the data before actually running any model. The main tasks of this phase are the following:

Selection of uncertain inputs. The uncertain inputs have to be defined a-priori. They may arise from different kind of sources, such as computational geometry, external loading, boundary conditions, material properties, etc. According to the experience and the degree of expertise on the problem, the selection of the uncertain inputs could be appropriate or not. Generally speaking, in case of doubt, it is better to include a variable than assuming that it is not influential.

Characterization of uncertainties in the inputs. Uncertain inputs can be represented by random variables. Classical categorization of uncertainties distinguishes between aleatoric and epistemic uncertainties [6]. The former are associated to the inherent variation of the parameters, cannot be reduced by additional physical or experimental knowledge, but they can serve to better categorize the uncertainty from probabilistic point of view. The quantification of irreducible uncertainties depends on the repetition of experiments to establish a statistically significant sample. Then, frequentist statistics can be used to estimate stan- 
dard deviations and confidence intervals, also, Bayesian theory offers a variety of methods and algorithms to calculate probability distributions by combining expert knowledge and data [7]. Often it is difficult to define experimental-based probability distributions for all the selected inputs and expert knowledge becomes fundamental. A common choice consists in exploring the model behaviour by assuming uniform distributions on the inputs, but it should be noted that changing the prescribed probabilities can lead to different conclusions. Then, running analysis with several distributions and comparing the results can be a useful exercise. The latter refers to deficiencies that result from model assumptions, missing physics, lack of complete information about the system. These uncertainties are often biased and they are typically less naturally defined in a probabilistic framework. Anyway the distinction between aleatoric and epistemic uncertainties is not always clear since lack of knowledge is relative and depends on current theory and experimental capability. Often in UQ applications, epistemic uncertainties are reformulated as aleatoric, where probabilistic analysis is applicable.

In the frame of numerical models, a particular class of epistemic uncertainties arises: the so-called model-form assumptions [8, 9]. All the models, from the coarser to more refined, contain choices which can compromise their reliability and predictiveness $[10,11]$. For example, there can be unquantified errors associated with the linear or non-linear behavior of a system, in the damping model [12], in the model of structural interfaces among components [13, 14].

Seeing at these choices in a probabilist framework they can be represented by discrete or categorical variables, which represent alternative plausible way of modelling or design choices, such as discrete selection of materials, components, or operational settings. Often they do not have a natural order or ranking. Unordered categorical variables are called nominal variables. While regression methodology is well established $[15,16]$, regarding categorical variables, the presence of both discrete and continuous probabilities distributions on uncertain inputs makes the UQ propagation and post-processing challenging. In fact, when the variables are continuous the response is assumed to vary smoothly, but discrete variables can compromise to have a continuous response behavior. As consequence, looking at the generation of a meta-model, due to the discontinuities, in the worst scenario a surrogate for each combination of discrete variables could be needed [17, 18]. Setting-up a scenario which attempts to probabilistically quantify the effects of arbitrary modelling assumptions or design choices on the QoIs requires care, and in any case, engineering knowledge, expertise, and possibly, experimental data 
for verification are fundamental.

Selection of Quantities of Interest. The QoIs, similarly to the uncertain inputs, have to be decided a priori. Their choice is a critical step because conclusions and decisions about a certain problem could change completely according to the defined QoIs.

\subsubsection{Uncertainties Propagation}

Uncertainty Propagation (UP) determines the distribution of the QoIs for a given distribution of the inputs. Due to the uncertainty in the inputs, the outputs of the model become uncertain as well, but neither the statistical moments nor a probability distribution are known a priory. They depend on the uncertainty of the parameters, which have to be propagated through the simulation.

Sampling Design. Non-intrusive methods sample the deterministic model at points in the multidimensional input parameter space, according some specific sampling technique, to create a cloud of result points, which can be further postprocessed to obtain sample-based statistics. The most common design is based on Montecarlo (MC) sampling, which relies on pure randomness. Due to randomness of the sampling process, some points can be clustered closely, while regions within the space may not contain any samples. Hence, especially when working in high dimensions, most researchers seem to agree that generic, near-uniform, distribution of the data sites is a better strategy. Several sampling strategies try to cover this need, such as the Latin Hypercube Design (LHD) and Low Discrepancy Design (LDD). LDD, in particular, generates a deterministic sequence of quasi-random numbers which fill uniformly the unit hypercube. The Halton low-discrepancy sequence [19] is well-known, easy to implement, and widely used in the literature. In the context of this thesis, Sobol's LDD is considered. It was the first digital sequence and operates in base-2 [20]. It has been found by several researchers, e.g. Saltelli et al. [21, chapter 2] and Pianosi [22], out-performing the classical MC and LHD, being, in average, more efficient and with a faster convergence rate. Anyway, when the computational demand of a single deterministic run is sufficiently high, sample-based approach based exclusively on the simulation model could be an unaffordable strategy. Hence, the need to first consider an efficient, smaller cloud of simulation points which allows to build a meta-model.

Meta-modelling. For complex systems, computationally demanding and where possibly a large set of inputs is considered uncertain, a sample-based approach based exclusively on a simulation model can be an unaffordable strategy. 
Hence, the need to first consider an efficient, smaller cloud of simulation points which allows to build a meta-model and then, by using it, complete the propagation of uncertainties and the sensitivity analysis.

The idea to replace a complex model by a computationally simple surrogate has gained increasing popularity in the past three decades [2, 23, 24]. These surrogates can be broadly categorized into two non-exclusive families: reductionbased and regression-based models. Their use is becoming widespread and they are making possible the numerical exploration of complex industrial applications $[5,14]$. The former methods are constructed by projecting states and distributed parameters onto a low-dimensional subspace, and, in some way, are still physics-based models. Common techniques to reduce the complexity of the large model are based on eigenfunctions, Proper Orthogonal Decomposition (POD), and Krylov subspaces [25, 26]. The latter aims to exploit the opaque nature of a complex phenomenon by constructing efficient input-output relations based on regression theory. The approximation coefficients are generally found by non-intrusive techniques that are easily adaptable to large-scale applications and general purpose software. Moreover, regression-based meta-models are nonparametric, in the sense that their coefficients do not have any concrete relation to the parameters involved in the problem. Hence, incorporating systematically the GSA is a useful and reliable way to quantify the influence of each parameter on the outputs. Regression type meta-models approximate the response of a complex system by a linear combination of simpler functions denoted as kernels. Polynomial Chaos Expansion (PCE) [27, 28], Kriging representation [29, 30] (also called Gaussian process) and Radial Kernels (RKs) [31, 32] (also known as Radial Basis Functions (RBFs)) are three of the most common methods to systematically construct this type of meta-models. All of them share assumptions about the continuity and the smoothness of the underlying and unknown multidimensional function which expresses the relationship between a set of stochastic inputs and a defined QoI. Evidently, no surrogate is truly universal [33, 34], and specific objectives together with problem type result in different optimal methods.

In the context of UQ and GSA, the PCE usually provides good approximations properties for relatively small problems with smooth response. Moreover, the close similarity between the PCE and the High Dimensional Model Representation (HDMR) proposed by Hoeffding provides a straightforward and computational inexpensive way to estimate the mean, the variance and Sobol's sensitivity indices [4, 35]. However, the performance of PCE deteriorates for medium/large problems and in the presence of non-smooth input-output mapping functions. In 
addition, using polynomials of high degrees might result in over-fitting [36].

A detailed study of Kriging methods can be found in [24, chapter 2, chapter $6]$ and in [32, chapter 5]. Although there are several different flavours of Kriging methods, in its simplest form it assumes that that a random field $Y$ has zero mean and a covariance kernel defined by a Gaussian-type function whose width can be controlled in each dimension by two hyper-parameters: a scale parameter $\theta$, and a smoothing exponent $p$. The Kriging method shows good predictiveness but it is computationally intensive due to the cost of estimating such parameters [24, 37]. In any case, the approach introduces the concept of anisotropy, and in fact Forrester [24] reports that the scale parameter $\theta$ can be considered as a measure of the correlation among different points for each dimension. Smaller values of $\theta$ (flatter kernels) imply similar behaviours across a specific dimension, while larger values of $\theta$ indicate more activity. By examining the values of $\theta$, one could qualitatively assess the relevance of all the variables and perhaps eliminate unimportant ones from future analyses.

In the end, the generation of a meta-model, or surrogate model, considers the computer simulations as a black-box which produces a set of outputs based on a given set of initial conditions and model parameters. The main task is employing a scattered data fitting approach to produce a surrogate for such computer simulations. Compared with the original one, the meta-model, expressing the relationship between the QoIs and the input variables, is considered to be computationally inexpensive. Evidently its generation requires a computer design, whose main objective is sampling the deterministic numerical model efficiently. That means to deal with the curse of dimensionality: the design should be able to effectively fill out the high-dimensional space and ensure an accurate solution of the scattered data fitting problem.

\subsubsection{Post-processing}

Post-processing activities regard fundamentally the stochastic characterization of the QoIs, which means to provide the statistics of the output density distributions. As result of the UP, either directly by the simulation model or by a computational cheaper auxiliary meta-model, a sample-based cloud of output points is obtained. Therefore, during a post-processing step, the QoIs can be stochastically characterized, which means to provide sample-based estimates of the mean, variance and their confidence intervals. This section includes also the GSA, whose objective is to rank uncertain inputs according their influence on the outputs. 
Global Sensitivity Analysis. GSA defines a qualitative and quantitative mapping between the input variables and the Quantities of Interest [21]. Sensitivity analyses are strictly related to the UP and they can be seen as complementary to each other: UP quantifying the uncertainty in the output of the model, while GSA focuses on apportioning the output's uncertainty to the different sources of uncertain parameters. Thus, GSA allows to select the relevant parameters in the model and to score them according to their effect on the QoIs.

The most popular GSA techniques are based on the decomposition of the variance's output probability distribution and allow the calculation of Sobol's Sensitivity Indices. They refer to Sobol', because the decomposition of the variance can be formulated analytically from the HDMR of Sobol' [38, 39]. Actually, Sobol in his 1993 paper made use of the functional decomposition presented by Hoeffding [40], to define the sensitivity indices and presents an efficient method to estimate them, which was further elaborated by Homma and Saltelli [41]. A comprehensive review can be found in $[42,43]$. From a practical point of view, special attention is paid to the first order and total order sensitivity indices, denoted respectively as $S_{i}^{I}$ and $S_{i}^{T}$, also called the main effects and the total effects.

If a model $Y$ is function of $N_{p}$ stochastic variables $X_{i}$ with $i=1, \ldots, N_{p}$, the main effects represent the measure of the contribution the parameter $X_{i}$ to the output variance, or equivalently, the expected percentage reduction of the output variance $V(Y)$ obtained when the uncertainty of input parameter $X_{i}$ is eliminated. The total effects represent the total contribution of an input parameter considering its individual effect and its interactions with all the other factors.

They are respectively defined as:

$$
\begin{gathered}
S_{i}^{I}=\frac{V_{X_{i}}\left[E_{X_{\sim i}}\left(Y \mid X_{i}\right)\right]}{V(Y)}=\frac{V(Y)-E_{X_{i}}\left[V_{X_{\sim i}}\left(Y \mid X_{i}\right)\right]}{V(Y)}, \\
S_{i}^{T}=\frac{E_{X \sim i}\left[V_{X_{i}}\left(Y \mid X_{i}\right)\right]}{V(Y)}=\frac{V(Y)-V_{X \sim i}\left[E_{X_{i}}\left(Y \mid X_{i}\right)\right]}{V(Y)} .
\end{gathered}
$$

This means that for non-additive models if all of the $V\left(X_{i}\right)$ terms are computed, then most likely $\sum_{i} V\left(X_{i}\right)$ would still be lower than the total $V(Y)$, because the difference $V(Y)-\sum_{i} V\left(X_{i}\right)$ is a measure of the impact of the interactions.

The popularity of the main and total effects is also due to the relatively easy closed-form algorithms, mostly proposed by Saltelli [21], who provides samplebased closed-form equations for their approximation. However, the simulations 
required to achieve reliable values of the indices can be rather large, which makes the direct application of these approaches quite unpractical for time-consuming models. In fact, the application of the re-sampling strategy leads to $N_{\text {run }}=$ $N_{s}\left(N_{p}+2\right)$ model evaluations, where $N_{s}$ can vary from a few hundreds to several thousands depending on the application (complexity of the model, input variables involved and their distributions, etc.).

Methods for reducing the computational burden have arisen. The most widely used are those based on the Fourier Amplitude Sensitivity Test (FAST) and those based on meta-modelling. FAST was first developed by Cukier in 1973 [44] for estimating the main effects and further extended (E-FAST) to the total effects by Saltelli in 1999 [45]. Meta-modelling plays also an important rule, because a limited set of runs is supposed to be sufficient for building a surrogate of the original model, and then this, computationally inexpensive, is used to complete the GSA. In addition, sensitivity indices have been employed as an effective tool for generating Reduced Order Methods (ROMs) [46], helping optimisation and reliability analysis. In fact, once the set of relevant parameters is selected, one can think to generate a more refined UQ process (UP, Meta-modelling, etc...) but only including those variables having the most significant impact on the response. Parameters belonging to this smaller set are often called Design Variables (DVs).

Additional post-processing of the sample based outputs can be used to draw conclusions about their distributions [47]. Many methods exist for testing whether a variable has a normal distribution. A quick visualization can be done by plotting an histogram of the QoIs variables and see their deviation from the theoretical bell curve distribution. Also, box-plots provide a very fast overview for the detection of non-normal samples. Box-plots draw a five-number summary of a random variable including its minimum, first quartile, median, third quartile and maximum. More refined evaluation of the deviation from a normal distribution can be obtained by quantile vs. quantile plot. It is used to plot theoretical quantiles, which follow a straight line for the normal distribution, against the actual quantiles of the variable of interest.

If the visualization techniques are not conclusive, statistical inference by hypothesis testing can give a more objective answer to whether a variable deviates significantly from a normal distribution. For example, the Kolmogorov-Smirnov (KS) [48] test gauges the goodness of fit of a given set of data to a theoretical distribution and computes the distances between the empirical and the theoretical distributions and defines the test statistic as the supremum of the set of 
those distances. An advantage of this is that the same approach can be used for comparing any distribution, not exclusively the normal one. If the observed data perfectly follow a Gaussian distribution, the value of the KS statistic will be zero. The P-Value is used to decide whether the difference is large enough to reject the null hypothesis: if the P-Value is higher than 0.05, a normal distribution is assumed. In practice, and in light of the Central Limit Theorem [49], when employing independent random variables, it is common to end up with results which follow approximately Gaussian distributions. In this case, the QoIs are completely specified by their mean and variance: that is, if those two things are known, one can figure out everything else about the distribution (probabilities, confidence intervals, etc.).

\subsection{Motivations and thesis' objectives}

Usually, in an industrial environment, the complexity of the computational models requires long runs and, in the end, the only efficient and realistic way to implement UQ and GSA processes passes through being able to build an efficient and predictive surrogate. Rigorous mathematical work can be found on surrogates but frequently their application is limited to analytical functions, suitable to demonstrate specific properties of the designed meta-model, but which, for example, do not present any constraint in the available number of simulations or do not need to be integrated in a wider industrial process. Often, Sobol's sensitivities have analytical expressions or can be estimated by sampling directly the anaylitical function and that allows the researcher to calculate the error of the approximation due to the meta-model, with accurancy.

In contrast, an industrial user seldom questions the performance of a metamodel, which is used as a black-box, and most parameters are just left to their default values. Often, some key aspects of the surrogate are not adequately considered. For example, it is common to use all the available simulations without taking care of how the model performs on unseen set of data. The error associated to the generation of the meta-model is not estimated in a rigorous way, and sometimes it is evaluated on the same set of data used for building the surrogate. When the GSA are completed no reference exists to understand how good the evaluation of the sensitivity indices is. Finally, based on the obtained outcomes, some decisions, which will affect directly the industrial process, the design and probably the direct cost of the products, are made. Paradoxically, in an industrial environment, where more refined results can make the difference, the quality is 
often penalized due to other constraints, such as timing, lack of experience, and costs.

The main objective of this thesis is to provide insight on UQ and GSA processes and explore in detail those aspects of meta-models which make them suitable for real applications. For this purpose, UQ, GSA and the generation of welldesigned meta-models, instrumental to complete both processes, are revisited by providing a balance between a rigorous mathematical approach and practical guidelines needed in an industrial environment. It is achieved by:

- Providing a clear description and an exhaustive literature review about UQ and GSA processes.

- Providing a perspective on Sobol's sensitivity indices and investigate Saltelli's reseampling approach using experimental results from a test campaign.

- Defining a rigorous framework for metamodels evaluation and selection, and providing a methodology to systematically construct quasi-optimal metamodels.

- Studying several applications and in particular two real industrial cases, which can be of relevant interest for the mechanical engineering community.

\subsection{Outline}

Motivated by the previous observations, chapter 2 reviews the Hoeffding's HDMR, how it leads to Sobol's variance decomposition and to the definition of sensitivity indices. A test campaign is presented in order to achieve an in-depth understanding of Sobol's sensitivities, which are formulated analytically first, and then used to understanding the convergence of the re-sampling algorithm proposed by Saltelli. The analysis takes into account three categorical variables and is completed by using exclusively the results from the test campaign. One relevant conclusion is that, in general, to complete the UQ process a model is absolutely required. Moreover, in case of expensive computational models, additionally, there is need to generate first a reliable and efficient meta-model.

Chapter 3 introduces the main concepts involved in building a meta-model and, in particular, studies the role of surrogates based on radial kernels in both the UQ and GSA processes. It is only by analyzing jointly both techniques that the real advantages and disadvantages of meta-modelling can be ascertained. A rigorous framework for the evaluation of the error associated to surrogate's generation is defined, in particular looking at its performance on unseen sets of data. 
In this context, the use of anisotropic kernels is introduced as an opportunity for obtaining improved performance, particularly when a limited set of model evaluations is available. In general, this type of kernels have not received much attention in the literature on approximation theory, apart from some isolated works $[50,51]$ which investigate their regularizing properties for surface reconstruction. Moreover, Fasshauer and McCourt [32] provide an interesting theoretical motivation for their potential use, which is used as inspiration for the novel approach presented in this thesis. A detailed methodology is proposed, which links anisotropic metrics with Sobol's sensitivities and can be used to systematically construct quasi-optimal meta-models. The qualitative connection between 'active dimensions' and relevant variables studied by Forrester [24] in virtue of the scale parameter $\theta$ of the Kriging method, in this thesis is revisited and quantified by defining a link between anistropic metric and Sobol's sensitivities. Anisotropic radial kernels can provide an extremely robust and cost-effective tool to complete the uncertainty propagation and study the sensitivity of complex systems and their stochastic response. As an example of that an analytical function, the well-known Sobol's $G$-function is studied by means of surrogates. However, where this thesis stands out most is in studying, with the same rigorous approach, two complex industrial cases. The first of them is presented in this chapter and considers Taylor's anvil impact test, which is one of the most common procedures to study the rate dependency of the plastic deformation in metals. To the author' best knowledge no literature exists which provides information about the relevance of the material parameters and their interaction with the impact's velocity. I provide the first UQ and GSA analysis which can help the parameter fitting for constitutive models, and guide the formulation of effective meta-models for other similar complex experimental setups.

Chapter 4 describes an industrial application in the context of non-linear structural vibrations. A nonlinear ROM, the Nonlinear Structural Dynamic Modifications Method (NL SDMM) [52, 53, 54] is presented as a reliable and efficient method to avoid the computational burden due to localized nonlinearities and is embedded in the UQ process. The study-case considers an aircraft component whose dynamic behavior is affected by free-plays interfaces, which are intrinsically uncertain. The NL SDMM, is presented in detail and used in conjunction with a PCE meta-model to provide a novel and pragmatic approach to evaluate the stochastic response of assembled structures with nonlinear uncertain interfaces. Also, the chapter briefly introduces the well known PCE and puts emphasis on the mathematical link between the coefficients of the expansion and the sen- 
sitivity indices. The main concepts are reviewed although in a non-exhaustive fashion.

The main conclusions are summarized in chapter 5 , together with some prospective avenues, opened in this thesis, that could be addressed in the future. 


\section{Chapter 2}

\section{A perspective on Sobol's sensitivities based on an experimental campaign}

\section{$2.1 \quad$ Introduction}

In this chapter Hoeffding's HDMR is introduced in order to explain the variance decomposition, to derive Sobol's sensitivity indices from it, and to present the re-sampling approach proposed by Saltelli to estimate them.

An experimental campaign is used to explore in depth and reformulate the presented concepts and methodologies. The test considers a beam supported by two springs with very low stiffness and studies how the first three natural modes of vibration vary by changing the number of lumped masses on the beam (see 2.3). The uncertain parameters are defined by the independent existence of three masses and the normal modes of the beam are obtained experimentally in a 2-level, 3-variables Full Factorial Design (FFD). Eight possible configurations are tested and the results are used to generate a polynomial regression model whose coefficients give indication about the importance of the variables. This regression model is used in order to formulate analytically the Sobol' sensitivities and to study the relationship between the Sobol's effects and the coefficient of determination in regression (R2). Finally, the re-sampling approach is evaluated in terms of needed sample size for having convergence to reliable estimates of the sensitivities. 


\subsection{High Dimensional Model Representation}

The method of Sobol' $[38,39]$ formulates analytically the variance decomposition from Hoeffding's HDMR [40]. Under the assumption of independent inputs, Hoeffding showed that a model output can be uniquely expressed as a sum of increasing dimension functions, where the integrals of every term with respect to any of its own variables must be zero. A consequence of these conditions is that the terms of the decomposition are mutually orthogonal. Later, Sobol' applied this decomposition to demonstrate that the global variance can be written as a sum of partial variances that quantify the sensitivity of a set of inputs on the model response.

To make these concepts more formal, let us consider a model represented by a function

$$
Y=f(X)=f\left(X_{1}, X_{2}, \ldots \ldots, X_{N_{p}}\right)
$$

where $Y$ is the univariate model output and $X=\left(X_{1}, X_{2}, \ldots, X_{N_{p}}\right)$ is a set of random independent parameters uniformly distributed within the unit hypercube, i.e, $X \in\left[\begin{array}{ll}0 & 1\end{array}\right]^{N_{p}}$. If $f(X)$ is integrable in $\left[\begin{array}{ll}0 & 1\end{array}\right]^{N_{p}}$, then it can be decomposed into a sum of functions of increasing dimensionality:

$$
f(X)=h_{0}+\sum_{i=1,2, \ldots, N_{p}} h_{i}\left(X_{i}\right)+\sum_{1 \leq i<j \leq N_{p}} h_{i j}\left(X_{i}, X_{j}\right)+\ldots+h_{12 \ldots N_{p}}\left(X_{1}, \ldots, X_{N_{p}}\right) .
$$

This decomposition is unique under the conditions that $h_{0}$ is a constant and the terms of the decomposition satisfy the equality

$$
\int_{[01]^{N_{u}}} h_{u}\left(X_{u}\right) d X_{k}=0
$$

where $u \subseteq 1,2, \ldots, N_{p}$ defines a subset $X_{u}$, of the $N_{p}$ variables and the index $k \subseteq u$. It implies that the functions are mutually orthogonal with respect to the $\mathbb{L}_{2}$ inner product and $h_{0}$ is equal to the expectation value of the output. Then 
Sobol's decomposition satisfies the equations:

$$
\begin{aligned}
& h_{0}=\int_{\left[\begin{array}{ll}
0 & 1
\end{array}\right]^{N_{p}}} f(X) d X=E(Y),
\end{aligned}
$$

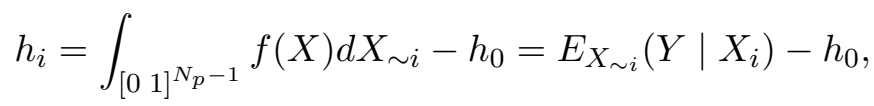

$$
\begin{aligned}
& h_{i j}=\int_{[01]^{N_{p}-2}} f(X) d X_{\sim i j}-h_{0}-h_{i}-h_{j}=E_{X_{\sim i j}}\left(Y \mid X_{i j} j\right)-h_{0}-h_{i}-h_{j} \text {, } \\
& h_{u}=\int_{[01]^{N_{p}-N_{u}}} f(X) d X_{\sim u}-h_{0}-\sum_{k} h_{k}=E_{X \sim u}\left(Y \mid X_{u}\right)-h_{0}-\sum_{k} h_{k},
\end{aligned}
$$

where the index $k$ spans the group of variables $X_{u}$.

Now, further assuming that $f(X)$ is square-integrable, the functional decomposition may be squared and integrated to give

$$
V(Y)=E\left(f(X)^{2}\right)-[E(f(X))]^{2}=\int_{[01]^{N_{p}}} f^{2}(X) d X-h_{0}^{2},
$$

and the partial variance associated with the summand $h_{u}$, within the group of variables $X_{u}$, with $X_{u} \subseteq X_{1}, X_{2}, \ldots, X_{N_{p}}$, is

$$
V_{u}=V\left(h_{u}\right)=E\left(h_{u}^{2}\right)-\left[E\left(h_{u}\right)\right]^{2}=\int_{[01]^{N_{u}}} h_{u}^{2}\left(X_{u}\right) d X_{u},
$$

because due to Eq.(2.3) the term $\left[E\left(h_{u}\right)\right]^{2}$ vanishes.

\subsubsection{Variance decomposition and sensitivity indices}

Eqs. (2.2), (2.5 and (2.6), finally lead to the decomposition of the variance as

$$
V(Y)=\sum_{i=1,2, \ldots, N_{p}} V_{i}+\sum_{1 \leq i<j \leq N_{p}} V_{i j}+\ldots+V_{12 \ldots N_{p}}
$$

where

$$
V_{i}=V_{X_{i}}\left[E_{X_{\sim i}}\left(Y \mid X_{i}\right)\right]
$$

is the conditional variance of variable $X_{i}$ and

$$
V_{i j}=V_{X_{i} X_{j}}\left[E_{X_{\sim i j}}\left(Y \mid X_{i} X_{j}\right)\right]-V_{i}-V_{j},
$$


is the variance due to the interaction between variables $X_{i}$ and $X_{j}, i \neq j$, and so on. Hence, in general, the variance associated with a group of variables $X_{u}$, with $X_{u} \subseteq X_{1}, X_{2}, \ldots, X_{N_{p}}$, is

$$
V_{u}=V_{X_{u}}\left[E_{X_{\sim u}}\left(Y \mid X_{u}\right)\right]-\sum_{k} V_{k}
$$

where the index $k$ spans the group of variables $X_{u}$.

From Eq. (2.7), dividing both sides of the identity by the total variance, the variance-based sensitivity indices are obtained:

$$
1=\sum_{i=1,2, \ldots, N_{p}} S_{i}+\sum_{1 \leq i<j \leq N_{p}} S_{i j}+S_{12 \ldots N_{p}}
$$

A sensitivity index associated with the $X_{u}$ group of variables is defined as

$$
S_{u}=\frac{V_{u}}{V(Y)}
$$

Higher-order sensitivity indices are usually not estimated directly because if the model consists of $N_{p}$ stochastic variables, the total number of indices (including the main ones) is $2^{N_{p}}-1$. Often the number of parameters, particularly for industrial applications, is very high and only the first order $S_{i}$ and total order effect $S_{i}^{T}$ are used. They are respectively defined as:

$$
\begin{gathered}
S_{i}=\frac{V_{X_{i}}\left[E_{X_{\sim i}}\left(Y \mid X_{i}\right)\right]}{V(Y)}=\frac{V(Y)-E_{X_{i}}\left[V_{X_{\sim i}}\left(Y \mid X_{i}\right)\right]}{V(Y)}, \\
S_{i}^{T}=\frac{E_{X \sim i}\left[V_{X_{i}}\left(Y \mid X_{i}\right)\right]}{V(Y)}=\frac{V(Y)-V_{X \sim i}\left[E_{X_{i}}\left(Y \mid X_{i}\right)\right]}{V(Y)} .
\end{gathered}
$$

The main effects represent the measure of the contribution of the parameter $X_{i}$ to the output variance, while the total effects represent the total contribution of an input parameter considering its individual effect and its interactions with all the other factors. The total effects is a compact measure which concentrates in one single term on all the interactions involving a given variable $X_{i}$. For example, with $N_{p}=3$, the total index associated with the variable $X_{1}$ is $S_{1}^{T}=$ $S_{1}+S_{12}+S_{13}+S_{123}$.

\subsubsection{Estimation of sensitivity indices}

For tractable functions, Sobol's indices above may be calculated analytically by evaluating the integrals in the decomposition. However, in the vast majority 
of cases they are estimated by the MC method. Often, and also in this thesis, the pure MC is replaced by more efficient LDD, such as the Sobol's sequence (see more details in section 1.2.2). Saltelli provides sample-based closed-form equations for their approximation[21, 55, 56, 57], which are summarized next.

Let $N_{s}$ be the sample size and $N_{p}$ the number of input random variables, the procedure is based on a resampling strategy which first generates two independent sample matrices $A$ and $B$, each one of size $N_{s} \times N_{p}$, and then builds $N_{p}$ further $N_{s} \times N_{p}$ matrices $A B_{i}$, for $i=1,2, \ldots, N_{p}$ such that the $i^{t h}$ column of $A B_{i}$ is equal to the $i^{\text {th }}$ column of $B$, and the remaining columns are from $A$.

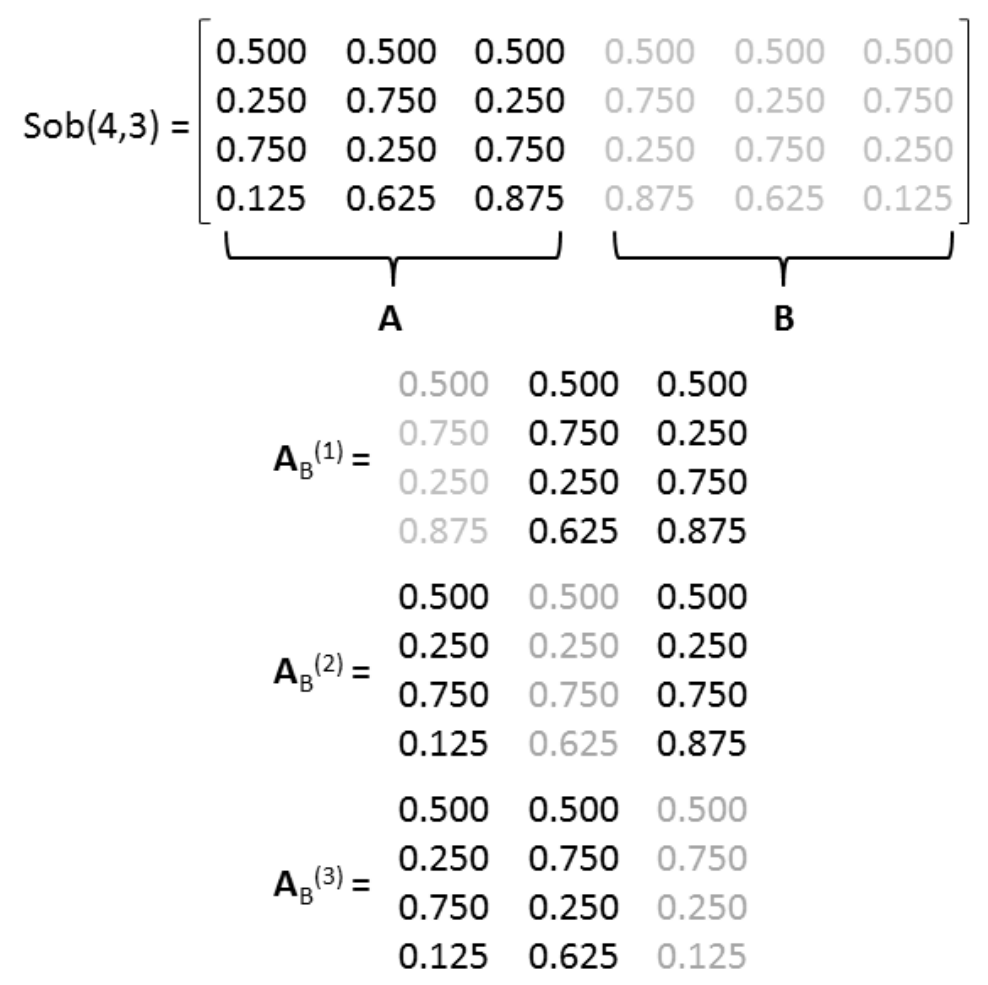

Figure 2.1: An example of construction of $A B_{i}$ matrices with $N_{p}=3$ and $N_{s}=4$. (source|Wikipedia)

Matrices $A, B$, and the $N_{p}$ matrices $A B_{i}$ in total require $N_{s}\left(N_{p}+2\right)$ model evaluations and the corresponding $f(A), f(B)$ and $f\left(A B_{i}\right)$ output values. Then, the sensitivity indices can be estimated as [57]

$$
V_{X_{i}}\left[E_{X_{\sim i}}\left(Y \mid X_{i}\right)\right]=\frac{1}{N_{s}} \sum_{j=1}^{N_{s}} f(B)_{j}\left(f\left(A B_{i}\right)_{j}-f(A)_{j}\right)
$$




$$
E_{X \sim i}\left[V_{X_{i}}\left(Y \mid X_{i}\right)\right]=\frac{1}{2 N_{s}} \sum_{j=1}^{N_{s}}\left(f\left(A B_{i}\right)_{j}-f(A)_{j}\right)^{2} .
$$

Saltelli's resampling approach together with Sobol's LDD are used to estimate the main and total effects throughout this thesis. Since the required dimension $N_{s}$ is often of the order of hundreds or thousands of runs, computational expense can quickly become a problem when the model takes a significant amount of time for a single run. In this thesis, the author relies on the creation of efficient and predictive meta-models to considerably reduce the computational cost.

\section{$2.3 \quad$ Experimental set-up}

The test campaign has been carried out in the laboratory of the Mechanical Engineering Department of the Universidad Politécnica de Madrid (UPM) (see figure 2.2). The beam's section nominal properties are presented in figure 2.3. Its length and weight (see table 2.1) have been measured and a uniform density of $2.91[\mathrm{~g} / \mathrm{cm} 3]$ has been determined.

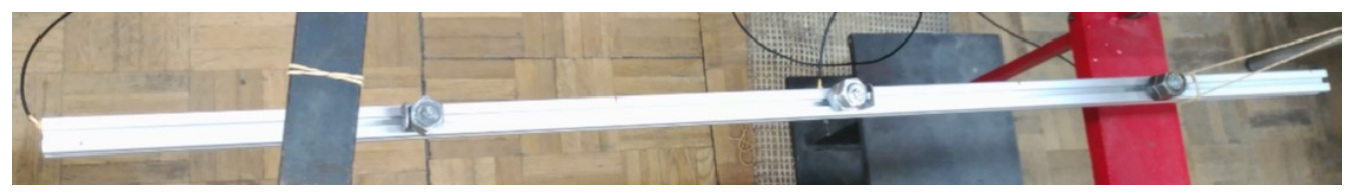

Figure 2.2: Beam test set-up at mechanical laboratory of the Mechanical Engineering department of the UPM. Three accelerometers are installed, two at the beam's ends and one in the centre in order to characterize the dynamic behaviour.

\section{$20 \times 20$}

$$
\begin{aligned}
& A=1,6 \mathrm{~cm}^{2} \\
& \mathrm{I}_{\mathrm{x}}=0,7 \mathrm{~cm} 4 \\
& \mathrm{I}_{\mathrm{y}}=0,7 \mathrm{~cm} 4 \\
& W_{x}=0,7 \mathrm{~cm}^{3} \\
& W_{y}=0,7 \mathrm{~cm} 3 \\
& m=0,4 \mathrm{~kg} / \mathrm{m}
\end{aligned}
$$

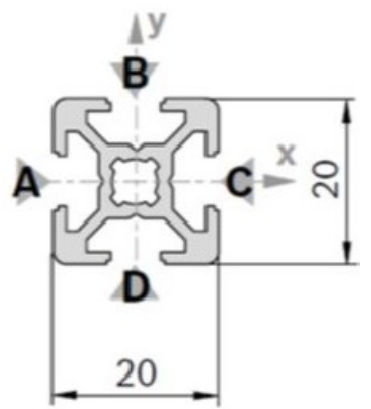

Figure 2.3: Beam section details. 
Three accelerometers are employed to characterize the first three free-free beam's natural modes of vibration: two of them are glued at the ends and one in the middle of the beam. Sensors' weights are measured and reported in table 2.2. An hammer test on the free-free structure is used to extract the modal properties: i.e. eigenvalues, eigenvectors and modal damping. The free-free conditions are ensured by hanging the structure by two springs with very low stiffness.

\begin{tabular}{ccc}
\hline Beam Length $[\mathrm{mm}]$ & Beam Weight $[\mathrm{mm}]$ & Density $\left[\mathrm{g} / \mathrm{cm}^{3}\right]$ \\
\hline 1000 & 465.2 & 2.91 \\
\hline
\end{tabular}

Table 2.1: Beam's properties.

\begin{tabular}{ccc}
\hline Sensor $1[g]$ & Sensor 2 $[g]$ & Sensor 3 $[g]$ \\
\hline 15.2 & 15.2 & 15.2 \\
\hline
\end{tabular}

Table 2.2: Masses of the accelerometers installed on the beam.

The test campaign is supported by a numerical model of the beam which used for the simulations. The model is calibrated according to the experimental data in the nominal case, i.e. without any additional masses but those of the sensors. Table 2.3 shows that the analytical results are in agreement with the experimental ones. Figure 2.4 shows the modal shapes.

\begin{tabular}{|c|c|c|c|}
\hline & TEST & FEM & \\
\hline Elastic Mode & Freq. $[\mathrm{Hz}]$ & Freq. $[\mathrm{Hz}]$ & Discrepancy \\
\hline 1 & 100.82 & 100.54 & $0 \%$ \\
\hline 2 & 283.56 & 285.53 & $1 \%$ \\
\hline 3 & 539.86 & 547.16 & $1 \%$ \\
\hline
\end{tabular}

Table 2.3: Comparison between experimental and numerical results.

Three dead-masses are considered in order to artificially introduce uncertainties in the system. The randomness lies in the fact that each mass may exist or not. Analytically, the presence or not of the mass belongs conceptually more to a model-form uncertainty, which can be described by a categorical variable. The weights of the masses are reported in table 2.4. Also, it is worth noting that 

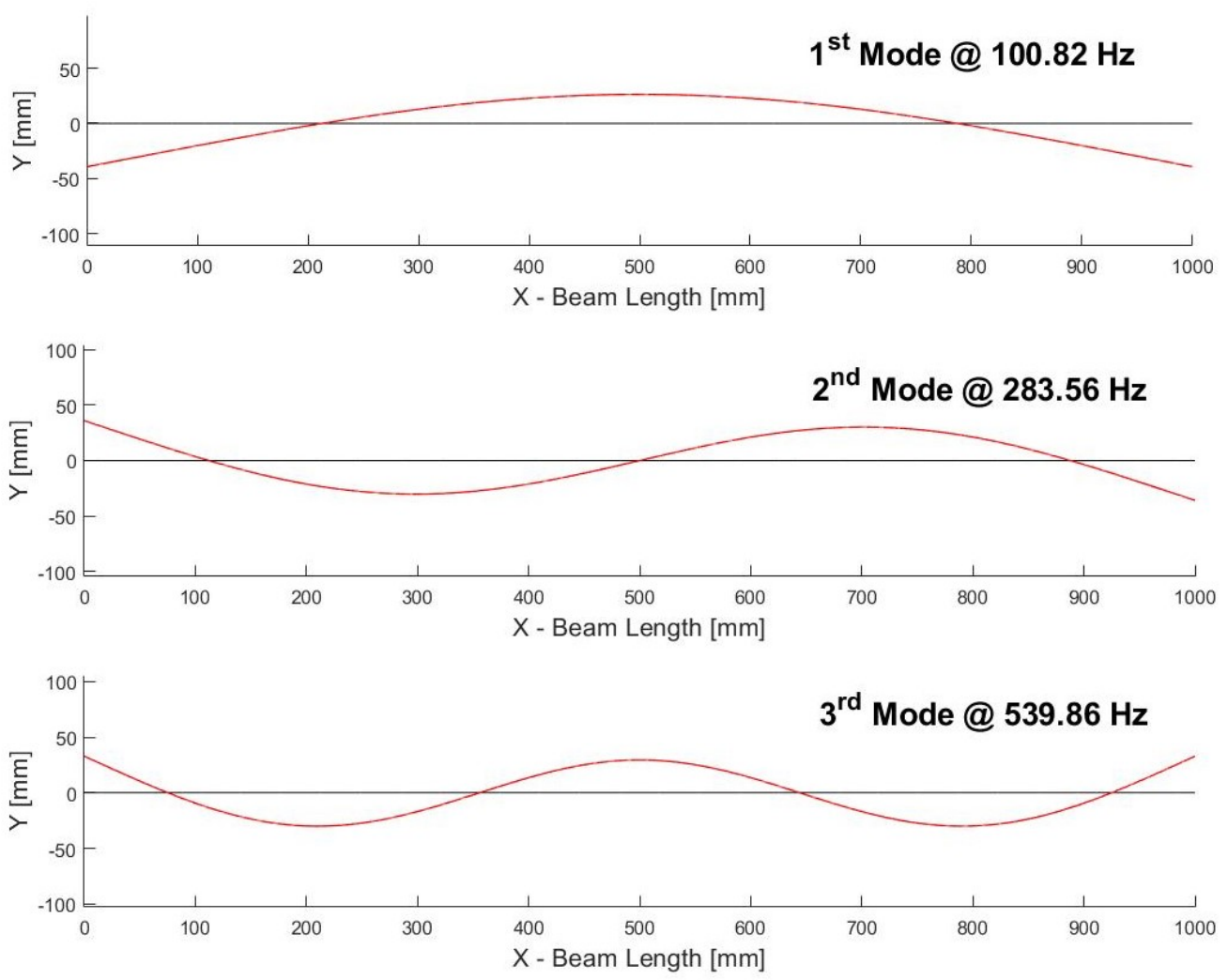

Figure 2.4: Beam's first three elastic modes. 
adding masses while keeping the remainder variable constant, reduced the natural frequencies. Hence, the natural frequencies of the beam without added masses (i.e. the nominal system configuration) are upper bounds of the distributions.

\begin{tabular}{ccc}
\hline Mass 1 $[g]$ & Mass 2 $[g]$ & Mass 3 $[g]$ \\
\hline 41.8 & 42.5 & 41.8 \\
\hline
\end{tabular}

Table 2.4: Dead-masses properties and prescribed probabilities.

\subsection{Factorial Design and Sensitivities}

In this section I am interested in studying how the uncertainty in having or not having the masses is propagated throughout the model and its implication on the statical inferences of the QoIs (i.e. the first three non-zero natural modes of the beam). Hence an experimental campaign, based in a 2-level, 3-variables (i.e, the categorical existence of masses) FFD, is used in order to capture the interactions among the parameters and avoid the misleading conclusions which could come varying One-factor-At-Time (OAT)). $2^{3} \mathrm{FFD}$ is shown in 2.5. It provides eight possible combinations, corresponding to the vertexes of a $3 D$-cube. The FFD effect is defined as the change in response produced by a change in the level of a factor.

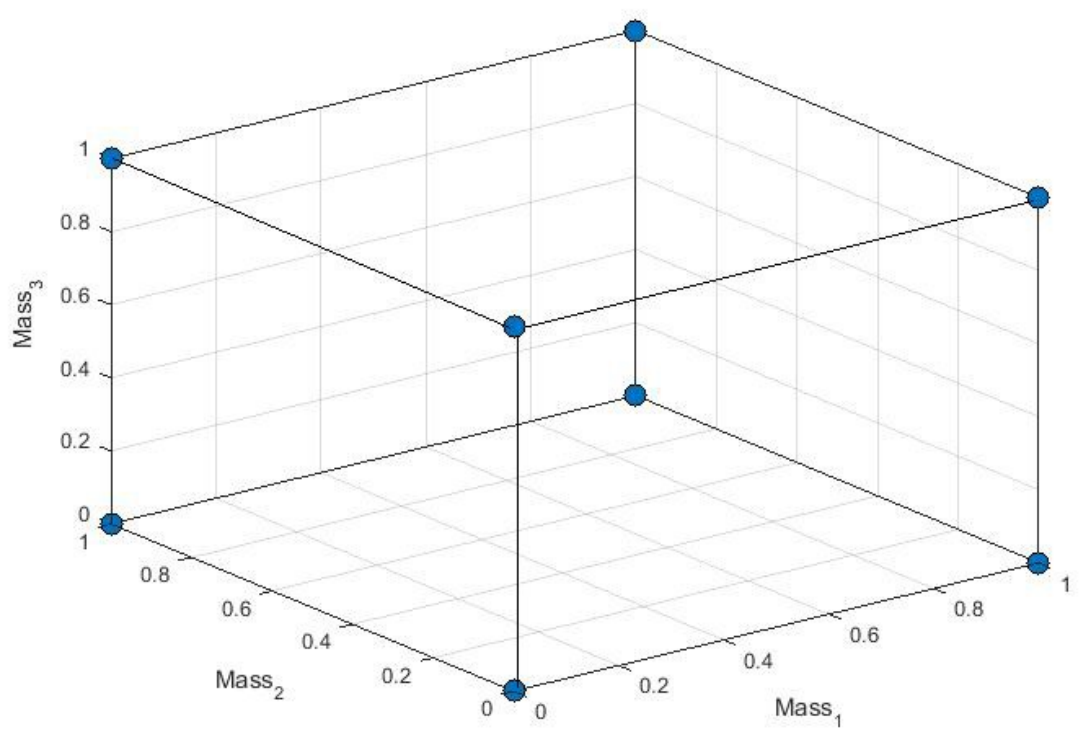

Figure 2.5: 2-level 3-variables FFD. 
Table 2.5 shows the eight combinations of the FFD. There are seven degree of freedom: three are associated with the main effects of parameters $\mathrm{A}, \mathrm{B}$ and $\mathrm{C}$, three with interactions $\mathrm{AB}, \mathrm{AC}$ and $\mathrm{BC}$ and finally, one with the interaction $\mathrm{ABC}$.

\begin{tabular}{ccccccc} 
Test & Mass 1(A) & Mass 2(B) & Mass 3(C) & Freq.1 $[\mathrm{Hz}]$ & Freq.2 $[\mathrm{Hz}]$ & Freq.3 $[\mathrm{Hz}]$ \\
\hline 1 & 0 & 0 & 0 & 100.54 & 285.53 & 547.15 \\
2 & 1 & 0 & 0 & 100.10 & 281.25 & 520.72 \\
3 & 0 & 1 & 0 & 95.48 & 285.53 & 515.61 \\
4 & 0 & 0 & 1 & 100.10 & 281.25 & 520.73 \\
5 & 1 & 1 & 0 & 94.88 & 281.22 & 492.45 \\
6 & 0 & 1 & 1 & 94.88 & 281.23 & 492.45 \\
7 & 1 & 0 & 1 & 99.65 & 277.42 & 497.77 \\
8 & 1 & 1 & 1 & 94.26 & 277.42 & 471.65 \\
\hline
\end{tabular}

Table 2.5: 2-level 3-variables FFD and frequency results.

A polynomial regression model is used in order to fit the experimental data. The regression includes the three variables and all their possible combinations:

$$
Y \simeq X \beta,
$$

with $X=\left[\begin{array}{lllllllll}1 & X_{1} & X_{2} & X_{3} & X_{1} X_{2} & X_{2} X_{3} & X_{1} X_{3} & X_{1} X_{2} X_{3}\end{array}\right]$.

The regression coefficients are estimated by means of a least square fitting, which has a closed-form solution:

$$
\beta=\left(X^{T} X\right)^{-1} X^{T} Y,
$$

where $X$ is assumed to have full column rank. The regression error is defined as

$$
\operatorname{Err}(\varepsilon)=\frac{\|Y-X \beta\|}{N_{\text {run }}\|X \beta\|}
$$

and in this case, where one has a full interpolation (i.e., the 8 unknowns, $\beta i$, are as many as the output points are), it is zero. The difference between regression and interpolation and the associated bias-variance dilemma is discussed in detail in Section 3.2.

In order to compare the effect of masses on the three frequencies, the values presented in table 2.5 are normalized with respect the value of the frequency in nominal conditions (i.e. those of test 1), see table 2.6. Table 2.7 presents the regression coefficients, sometimes called simply 'regressors'. Because of an explicit 
polynomial regression is employed, the value of the coefficients is an indication of on the relevance of the variables' contribution and this is the reason why the regressors are sometimes called 'effects'.

Coherently with the modal shape presented in Figure 2.4, one can observe that the presence of the second mass has the highest relevance for the first frequency, while its effect is negligible for the second mode. In fact, the center of the beam is a nodal point i.e. a fixed point, with respect to the second modal shape. For the third frequency one can see that all the three masses have similar contributions. The coefficient $\beta_{0}$ returns the value of the frequencies in nominal conditions. Once the masses are attached to the beam, these nominal values decrease according to the remaining coefficients $\beta_{i}$ with $i=1,2, \ldots, 7$.

\begin{tabular}{ccc} 
Freq.1/Freq.1,run1 & Freq.2/Freq.2,run1 & Freq.3/Freq.3,run1 \\
\hline 1.000 & 1.000 & 1.000 \\
0.996 & 0.985 & 0.952 \\
0.950 & 1.000 & 0.942 \\
0.996 & 0.985 & 0.952 \\
0.944 & 0.985 & 0.900 \\
0.944 & 0.985 & 0.900 \\
0.991 & 0.972 & 0.910 \\
0.938 & 0.972 & 0.862 \\
\hline
\end{tabular}

Table 2.6: Normalized values of frequencies in 2-level FFD.

\begin{tabular}{ccccc} 
& & Freq.1 & Freq.2 & Freq.3 \\
\hline 1 & $\beta_{0}$ & 1.00 & 1.00 & 1.00 \\
$X_{1}$ & $\beta_{1}$ & $-4.35 \times 10^{-3}$ & $-1.50 \times 10^{-2}$ & $-4.83 \times 10^{-2}$ \\
$X_{2}$ & $\beta_{2}$ & $-5.03 \times 10^{-2}$ & $-8.88 \times 10^{-6}$ & $-5.77 \times 10^{-2}$ \\
$X_{3}$ & $\beta_{3}$ & $-4.35 \times 10^{-3}$ & $-1.50 \times 10^{-2}$ & $-4.83 \times 10^{-2}$ \\
$X_{1} X_{2}$ & $\beta_{4}$ & $-1.66 \times 10^{-3}$ & $-7.83 \times 10^{-5}$ & $5.99 \times 10^{-3}$ \\
$X_{2} X_{3}$ & $\beta_{5}$ & $-1.65 \times 10^{-3}$ & $-6.94 \times 10^{-5}$ & $5.97 \times 10^{-3}$ \\
$X_{1} X_{3}$ & $\beta_{6}$ & $-6.27 \times 10^{-5}$ & $1.60 \times 10^{-3}$ & $6.34 \times 10^{-3}$ \\
$X_{1} X_{2} X_{3}$ & $\beta_{7}$ & $-6.97 \times 10^{-5}$ & $1.53 \times 10^{-4}$ & $-2.04 \times 10^{-3}$ \\
\hline
\end{tabular}

Table 2.7: Regression coefficients in 2-level FFD.

\subsubsection{Analytical evaluation of Sobol's indices}

In this section, the experimental data are analyzed again in the light of Sobol's indices. Because they are based on the decomposition of the variance, 
one needs to assign a probability to all the parameters involved. One can think that the existence of these masses is probabilistically defined by a Bernoulli distribution $X \sim \mathcal{B}(p)$ whose probability density function is

$$
f(x)=p^{x}(1-p)^{1-x}
$$

which takes the vale $x=1$ in case of the probability to success, $p$, and values $x=0$ in case of the probability to fail, $1-p$. This function is shown in table 2.8. They can be seen as nominal variables which produce a qualitative change in the system's model. The expectation is

$$
\mu(X)=p,
$$

and the variance is

$$
\sigma^{2}(X)=p(1-p)
$$

\begin{tabular}{ccc}
\hline Mass 1 $[g]$ & Mass 2 $[g]$ & Mass 3 $[g]$ \\
\hline 41.8 & 42.5 & 41.8 \\
$X_{1} \sim \mathcal{B}(p)$ & $X_{2} \sim \mathcal{B}(p)$ & $X_{3} \sim \mathcal{B}(p)$ \\
\hline
\end{tabular}

Table 2.8: Prescribed probabilities on dead-masses existence.

By using the regression model given by Eq. (2.17), where now the regression coefficients $\beta$ are known, the expected value is

$$
E(Y)=p \sum_{i=1}^{3} \beta_{i}+p^{2} \sum_{i=4}^{6} \beta_{i}+p^{3} \beta_{7}
$$

and total variance is

$$
V(Y)=p(1-p) \sum_{i=1}^{3} \beta_{i}^{2}+p^{2}\left(1-p^{2}\right) \sum_{i=4}^{6} \beta_{i}^{2}+p^{2}(1+2 p) \beta_{7}^{2} .
$$

Eq. (2.24) is obtained by considering that given $N$ independent random variables

$$
\sigma^{2}\left(X_{1} \cdot X_{2} \cdot \ldots \cdot X_{N}\right)=\prod_{i=1}^{N}\left(\sigma^{2}\left(X_{i}\right)+\left[\mu\left(X_{i}\right)\right]^{2}\right)-\prod_{i=1}^{N}\left[\mu\left(X_{i}\right)\right]^{2} .
$$

In this study, the three nominal variables are described by the same Bernoulli 
distribution with mean:

$$
\mu\left(X_{1}\right)=\mu\left(X_{2}\right)=\mu\left(X_{3}\right)=p=0.5,
$$

and variance

$$
\sigma^{2}\left(X_{1}\right)=\sigma^{2}\left(X_{2}\right)=\sigma^{2}\left(X_{3}\right)=p(1-p)=0.25
$$

and then one obtains

$$
\begin{gathered}
\sigma^{2}\left(X_{i} \cdot X_{j}\right)=\sigma^{2}\left(X^{2}\right)=p^{2}\left(1-p^{2}\right)=0.1875, \\
\sigma^{2}\left(X_{1} \cdot X_{2} \cdot X_{3}\right)=\sigma^{2}\left(X^{3}\right)=p^{2}(1+2 p)=0.5,
\end{gathered}
$$

for $i, j=1,2,3$ with $i<j$.

In the light of Eqs. (2.10), (2.12) and (2.24), one can obtain analytically all the Sobol's indices. As an example of it, the first effect $S_{1}$ is estimated. First the output expectation by considering all the independent random variables but $X_{1}$, is calculated. Hence, $X_{1}$ is treated as a non-random variable, which is equivalent to saying that the uncertainty on parameter $X_{1}$ has been eliminated:

$$
\begin{array}{r}
E_{X_{\sim 1}}\left(Y \mid X_{1}\right)=\beta_{0}+\beta_{1} X_{1}+\beta_{2} \mu\left(X_{2}\right)+\beta_{3} \mu\left(X_{3}\right)+\beta_{4} \mu\left(X_{3}\right) X_{1}+ \\
\beta_{5} \mu\left(X_{2}\right) \mu\left(X_{3}\right)+\beta_{6} \mu\left(X_{3}\right) X_{1}+\beta_{7} \mu\left(X_{1}\right) \mu\left(X_{2}\right) X_{1}= \\
\left(\beta_{0}+\beta_{2} p+\beta_{3} p+\beta_{5} p^{2}\right)+\left(\beta_{1}+\beta_{4} p+\beta_{6} p+\beta_{7} p^{2}\right) X_{1},
\end{array}
$$

and then the expected percentage reduction of the output variance $V(Y)$ due to the input parameter $X_{i}$ when all the other parameters have been fixed to their mean value:

$$
V\left(X_{1}\right)=V_{X_{1}}\left[E_{X_{\sim 1}}\left(Y \mid X_{1}\right)\right]=\left(\beta_{1}^{2}+\beta_{4}^{2} p^{2}+\beta_{6}^{2} p^{2}+\beta_{7}^{2} p^{4}\right) \sigma^{2}\left(X_{1}\right),
$$

where $\sigma^{2}\left(X_{1}\right)$ is given by Eq. (2.22). The main effect $S_{1}$, is

$$
S_{1}=\frac{\left(\beta_{1}^{2}+\beta_{4}^{2} p^{2}+\beta_{6}^{2} p^{2}+\beta_{7}^{2} p^{4}\right) p(1-p)}{p(1-p) \sum_{i=1}^{3} \beta_{i}^{2}+p^{2}\left(1-p^{2}\right) \sum_{i=4}^{6} \beta_{i}^{2}+p^{2}(1+2 p) \beta_{7}^{2}},
$$

with $p=0.5$ and the $\beta$ given in table 2.7 .

In a similar way the other variance terms and their related sensitivities are evaluated and shown in table 2.9. The regression coefficients are known (see table 2.7). The results are aligned with the qualitative importance given by the regression coefficients but now this relevance is quantified by means of the total 
variance decomposition. One can affirm that the main effects cover about the $99 \%$ of the total output variance and consequently all the interactions are negligible. Additionally, as showed in Fig. 2.6, for each mode of vibration, the masses whose presence affects the frequencies the most are completely clear.

\begin{tabular}{cc}
\hline$V\left(X_{1}\right)$ & $\left(\beta_{1}^{2}+\beta_{4}^{2} p^{2}+\beta_{6}^{2} p^{2}+\beta_{7}^{2} p^{4}\right) \sigma^{2}\left(X_{1}\right)$ \\
$V\left(X_{2}\right)$ & $\left(\beta_{2}^{2}+\beta_{4}^{2} p^{2}+\beta_{5}^{2} p^{2}+\beta_{7}^{2} p^{4}\right) \sigma^{2}\left(X_{2}\right)$ \\
$V\left(X_{3}\right)$ & $\left(\beta_{3}^{2}+\beta_{5}^{2} p^{2}+\beta_{6}^{2} p^{2}+\beta_{7}^{2} p^{4}\right) \sigma^{2}\left(X_{3}\right)$ \\
& \\
\hline$V\left(X_{1}, X_{2}\right)$ & $\left(\beta_{1}^{2}+\beta_{6}^{2} p^{2}\right) \sigma^{2}\left(X_{1}\right)+\left(\beta_{2}^{2}+\beta_{5}^{2} p^{2}\right) \sigma^{2}\left(X_{2}\right)+$ \\
& $\left(\beta_{4}^{2}+\beta_{7}^{2} p^{2}\right) \sigma^{2}\left(X_{1} \cdot X_{2}\right)-V\left(X_{1}\right)-V\left(X_{2}\right)$ \\
$V\left(X_{2}, X_{3}\right)$ & $\left(\beta_{2}^{2}+\beta_{4}^{2} p^{2}\right) \sigma^{2}\left(X_{2}\right)+\left(\beta_{3}^{2}+\beta_{6}^{2} p^{2}\right) \sigma^{2}\left(X_{3}\right)+$ \\
& $\left(\beta_{5}^{2}+\beta_{7}^{2} p^{2}\right) \sigma^{2}\left(X_{2} \cdot X_{3}\right)-V\left(X_{2}\right)-V\left(X_{3}\right)$ \\
$V\left(X_{1}, X_{3}\right)$ & $\left(\beta_{1}^{2}+\beta_{4}^{2} p^{2}\right) \sigma^{2}\left(X_{1}\right)+\left(\beta_{3}^{2}+\beta_{5}^{2} p^{2}\right) \sigma^{2}\left(X_{3}\right)+$ \\
& $\left(\beta_{6}^{2}+\beta_{7}^{2} p^{2}\right) \sigma^{2}\left(X_{1} \cdot X_{3}\right)-V\left(X_{1}\right)-V\left(X_{3}\right)$ \\
\hline$V\left(X_{1}, X_{2}, X_{3}\right)$ & $V(Y)-\left(V\left(X_{1}\right)+V\left(X_{2}\right)+V\left(X_{3}\right)+\right.$ \\
& $\left.V\left(X_{1}, X_{2}\right)+V\left(X_{2}, X_{3}\right)+V\left(X_{1}, X_{3}\right)\right)$ \\
\hline
\end{tabular}

Table 2.9: Decomposition of the total variance for the three masses problem. In this exercise $\sigma^{2}\left(X_{i}\right)=p(1-p)=0.25$, for $i=1,2,3$ and $p=0.5$. The term $\sigma^{2}\left(X_{i} \cdot X_{j}\right)=p^{2}\left(1-p^{2}\right)=0.1875$, for $i, j=1,2,3$ with $i<j$. These formulas are still valid for a different value of $p$ but one has to consider that by changing $p$, the output distribution changes accordingly and then the $\beta$ coefficients need to be re-calculated by a new regression. 


\begin{tabular}{cccc} 
Sobol's Indices & Freq.1 & Freq.2 & Freq.3 \\
\hline$S_{1}$ & $0.77 \%$ & $49.95 \%$ & $29.09 \%$ \\
$S_{2}$ & $98.41 \%$ & $0.00 \%$ & $41.36 \%$ \\
$S_{3}$ & $0.76 \%$ & $49.90 \%$ & $29.11 \%$ \\
\hline$S_{12}$ & $0.03 \%$ & $0.00 \%$ & $0.11 \%$ \\
$S_{23}$ & $0.03 \%$ & $0.00 \%$ & $0.11 \%$ \\
$S_{13}$ & $0.00 \%$ & $0.14 \%$ & $0.13 \%$ \\
$S_{123}$ & $0.00 \%$ & $0.01 \%$ & $0.08 \%$ \\
\hline$S_{1}^{T}$ & $0.80 \%$ & $50.10 \%$ & $29.42 \%$ \\
$S_{2}^{T}$ & $98.47 \%$ & $0.01 \%$ & $41.67 \%$ \\
$S_{3}^{T}$ & $0.79 \%$ & $50.05 \%$ & $29.44 \%$ \\
\hline
\end{tabular}

Table 2.10: Sobol's sensitivities obtained by recursive analytical formulas.

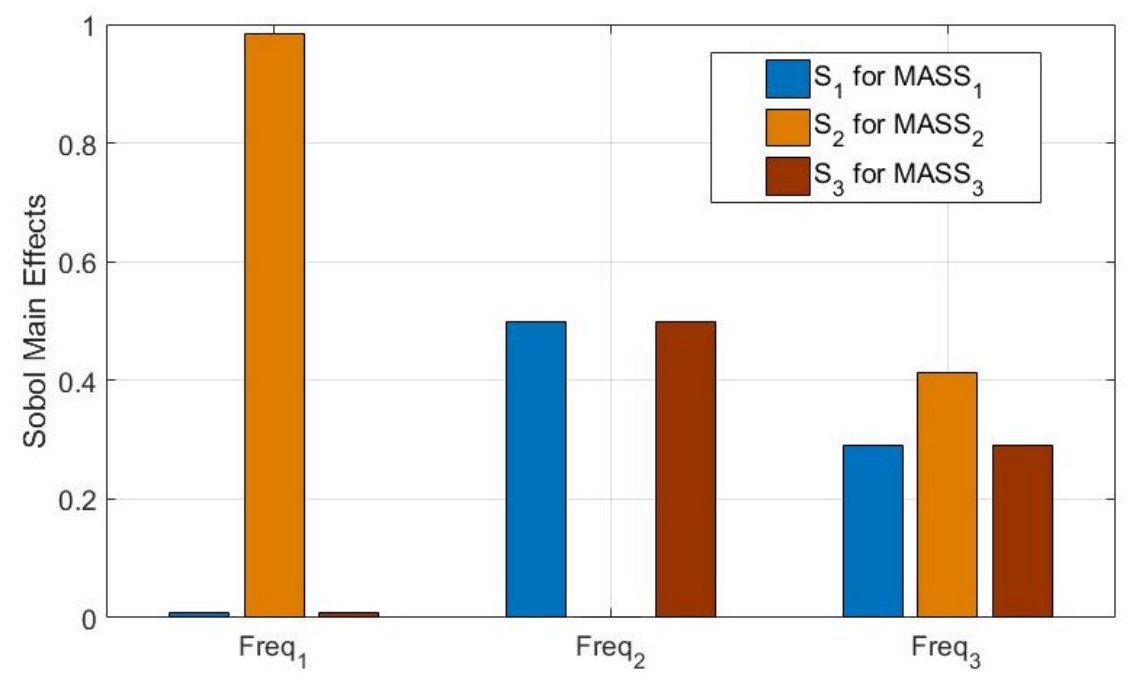

Figure 2.6: Sobol's main effects for first three frequencies.

\subsubsection{Estimation of Sobol's indices by the coefficient of determi- nation in regression}

Let us reconsider the Eq. (2.30), written as

$$
\begin{gathered}
E_{X \sim 1}\left(Y \mid X_{1}\right)=\left(\beta_{0}+\beta_{2} p+\beta_{3} p+\beta_{5} p^{2}\right)+ \\
\left(\beta_{1}+\beta_{4} p+\beta_{6} p+\beta_{7} p^{2}\right) X_{1}=\beta_{0}^{I}+\beta_{1}^{I} X_{1} .
\end{gathered}
$$

It means that the result of Eq. (2.33) can be estimated by a linear regression on $Y$ where only the parameter $X_{1}$ is considered. Let us consider the coefficient of 
determination in regression $\mathrm{R} 2$, which is a statistical measure of how well the regression predictions approximate the real data points. An R2 of 1 indicates that the regression predictions perfectly fit the data. ${ }^{1} \mathrm{R} 2$ is often interpreted as the proportion of response variation explained by the regressors in the model, hence the R2 given by the regression model in Eq. (2.33) is an estimate of the Sobol's main index $S_{1}$. In general the conditional expectation $E_{X_{\sim u}}\left(Y \mid X_{u}\right)$ due to a group of variables $X_{u}$, with $X_{u} \subseteq 1,2, . ., N_{p}$ can be estimated by the regression model $X_{u} \beta$. The results are presented in table 2.11. Figure 2.7 compares these values with those obtained analytically by close-form formula. The values obtained by the R2 regression approach are a very good estimates of the Sobol's effects.

\begin{tabular}{cccc} 
Sobol's Indices & Freq. 1 & Freq. 2 & Freq. 3 \\
\hline$S_{1}$ & $1.00 \%$ & $49.93 \%$ & $28.49 \%$ \\
$S_{2}$ & $97.96 \%$ & $0.00 \%$ & $42.69 \%$ \\
$S_{3}$ & $0.99 \%$ & $49.89 \%$ & $28.51 \%$ \\
\hline$S_{12}$ & $0.03 \%$ & $0.00 \%$ & $0.10 \%$ \\
$S_{23}$ & $0.03 \%$ & $0.00 \%$ & $0.10 \%$ \\
$S_{13}$ & $0.00 \%$ & $0.17 \%$ & $0.11 \%$ \\
$S_{123}$ & $0.00 \%$ & $0.00 \%$ & $0.00 \%$ \\
\hline$S_{1}^{T}$ & $1.02 \%$ & $50.11 \%$ & $28.71 \%$ \\
$S_{2}^{T}$ & $98.01 \%$ & $0.00 \%$ & $42.88 \%$ \\
$S_{3}^{T}$ & $1.02 \%$ & $50.07 \%$ & $28.72 \%$ \\
\hline
\end{tabular}

Table 2.11: Sobol's sensitivities obtained by recursive regression.

\footnotetext{
${ }^{1}$ It is defined as

$$
R^{2}=1-\frac{S S_{r e s}}{S S_{t o t}}
$$

where $S S_{\text {res }}=\sum_{i=1}^{N_{s}}\left(Y_{i}-X \beta\right)^{2}$ and $S S_{t o t}=\sum_{i=1}^{N_{s}}\left(Y_{i}-E(Y)\right)^{2}$.
} 


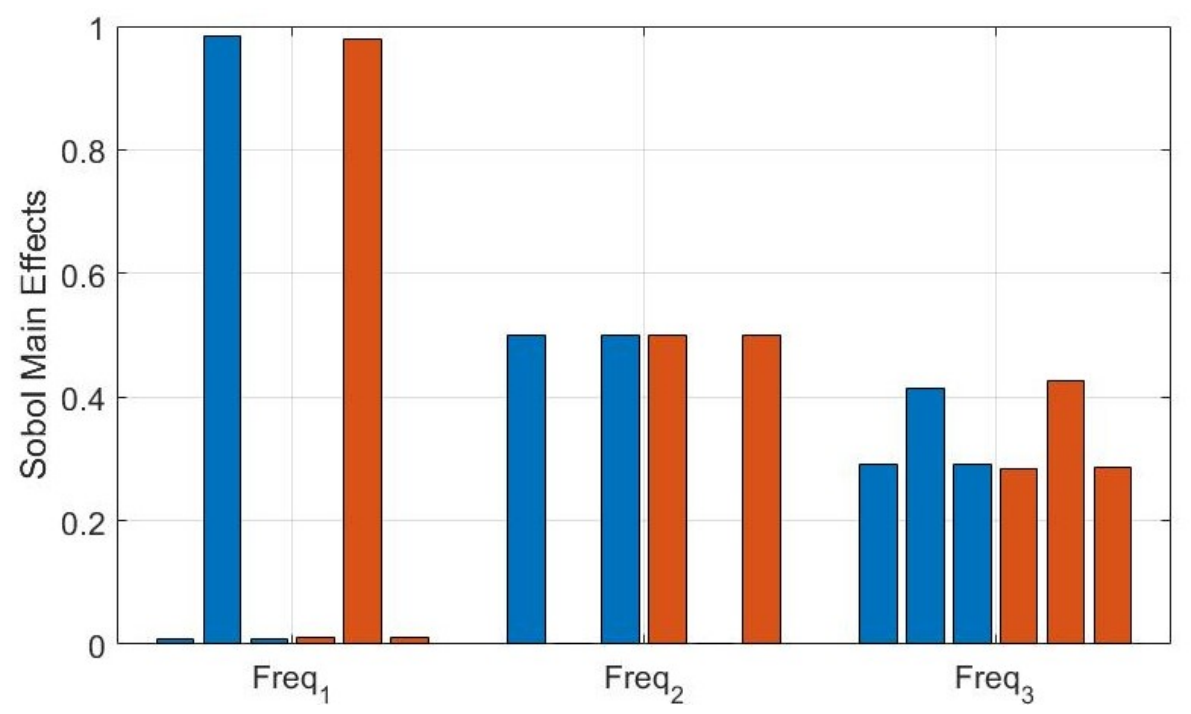

Figure 2.7: Comparison between the main effects for the first three frequencies. The first group (blue bars) takes in consideration the $S_{i}$ calculated analytically by close-form formula. The second group (orange bars) takes in consideration the $S_{i}$ estimated by the $R^{2}$ regression approach. The differences are negligible.

\subsubsection{Estimation of Sobol's indices by Low Discrepancy Design}

In this section, the approach presented in section 2.2.2 is used for the estimation of the main and total effects. Table 2.12 presents the results, which are in very good agreement with the analytical values, as shown in figure 2.10. In any case, to get reliable estimation of the indices a high number of samples is required. Figures 2.8 for the main indices and 2.9 for the total ones, show the convergence of the results.

In my estimation, a sample of size $N_{s}=2048$ is used, which is much higher than the 8 points used in sections 2.4 .1 and 2.4.2. In this case, since binomial variables are used, there is no need of a computational model because the 2048 sample are just a random repetition (generate anyway with the Sobol's LDD) of the first eight cases. When continuous and discrete random variables are involved, a model is essential to complete the UQ process. Additionally, particularly in case of expensive computational model, there is the evident need to generate first a reliable and efficient meta-model. 


\begin{tabular}{cccc} 
Sobol's Indices & Freq. 1 & Freq. 2 & Freq. 3 \\
\hline$S 1$ & $1.01 \%$ & $50.01 \%$ & $28.51 \%$ \\
$S 2$ & $98.42 \%$ & $0.01 \%$ & $42.83 \%$ \\
$S 3$ & $0.93 \%$ & $49.03 \%$ & $28.38 \%$ \\
\hline$S 1^{T}$ & $1.02 \%$ & $50.07 \%$ & $28.71 \%$ \\
$S 2^{T}$ & $98.00 \%$ & $0.00 \%$ & $42.88 \%$ \\
$S 3^{T}$ & $1.02 \%$ & $50.10 \%$ & $28.72 \%$ \\
\hline
\end{tabular}

Table 2.12: Sobol's sensitivities obtained by Saltelli approach.

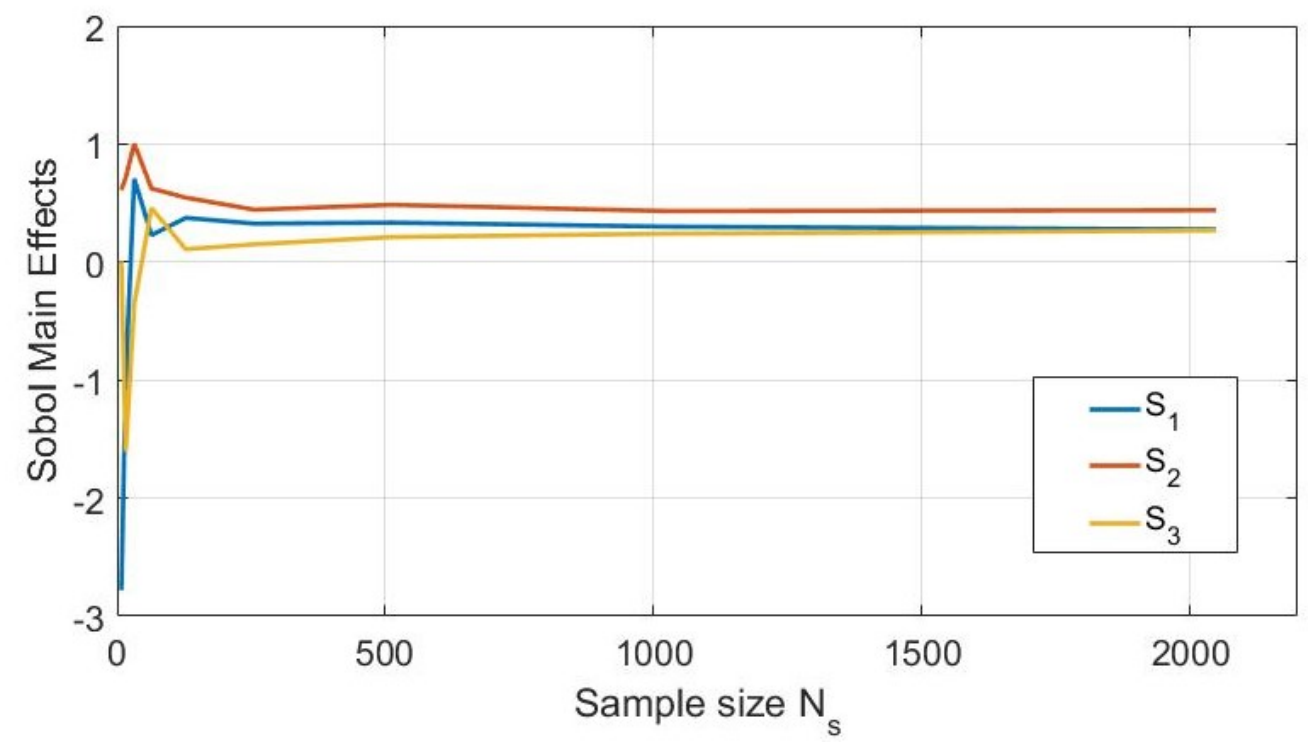

Figure 2.8: Convergence of Sobol's main effects vs. sample size. The picture shows the case of the third output frequency. Because of Saltelli approach is based on Sobol' LDD re-sampling matrices, an high number of samples is required to achieve the convergence and consequently a good estimation of the indices.

\subsection{Conclusions}

Sobol's sensitivities and their estimation by the re-sampling approach proposed by Saltelli are two key aspects of this thesis. In this chapter both methods are reviewed in the light of an experimental campaign which considers the first three natural modes of a beam. A 2-level, 3-variables FFD is used to combine three categorical variables: the potential presence of three lumped masses. From a statistical point of view these variables can be successfully described by a Bernoulli distribution. 


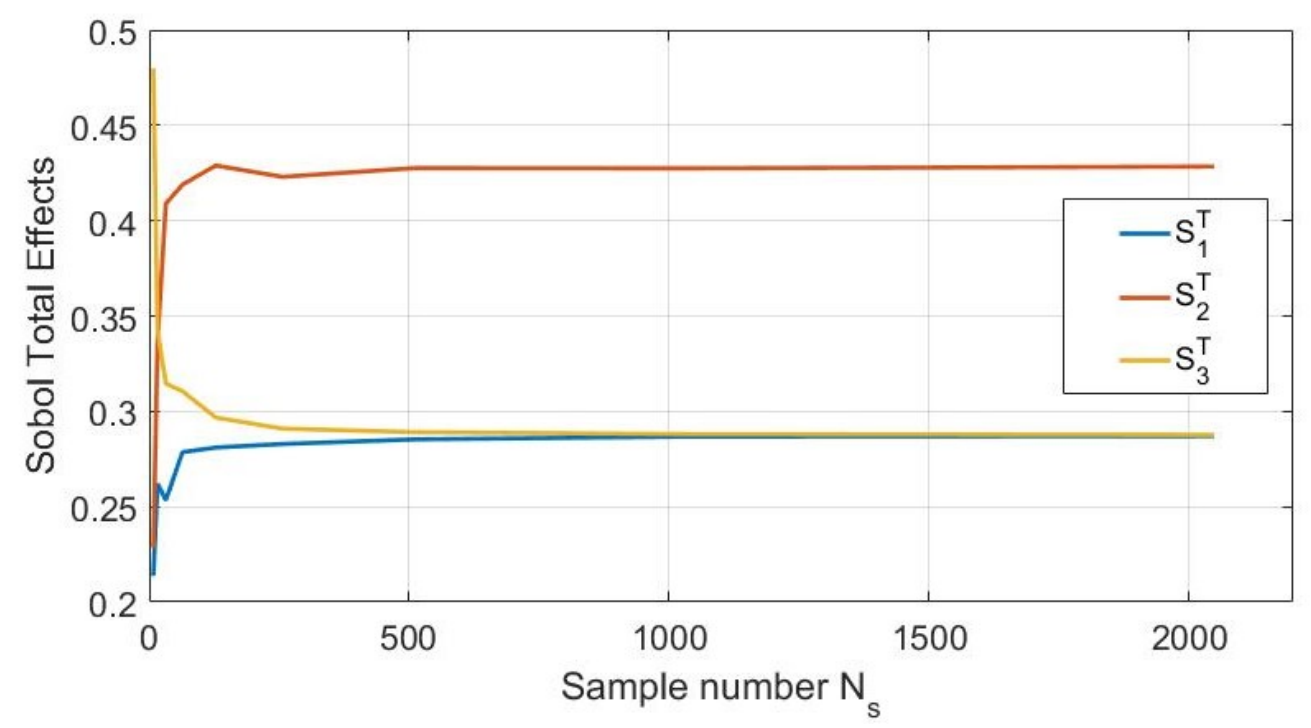

Figure 2.9: Convergence of Sobol's total effects vs. sample size. The picture shows the case of the third output frequency. Because of Saltelli approach is based on MC re-sampling matrices, an high number of samples is required to achieve the convergence and consequently a good estimation of the indices.

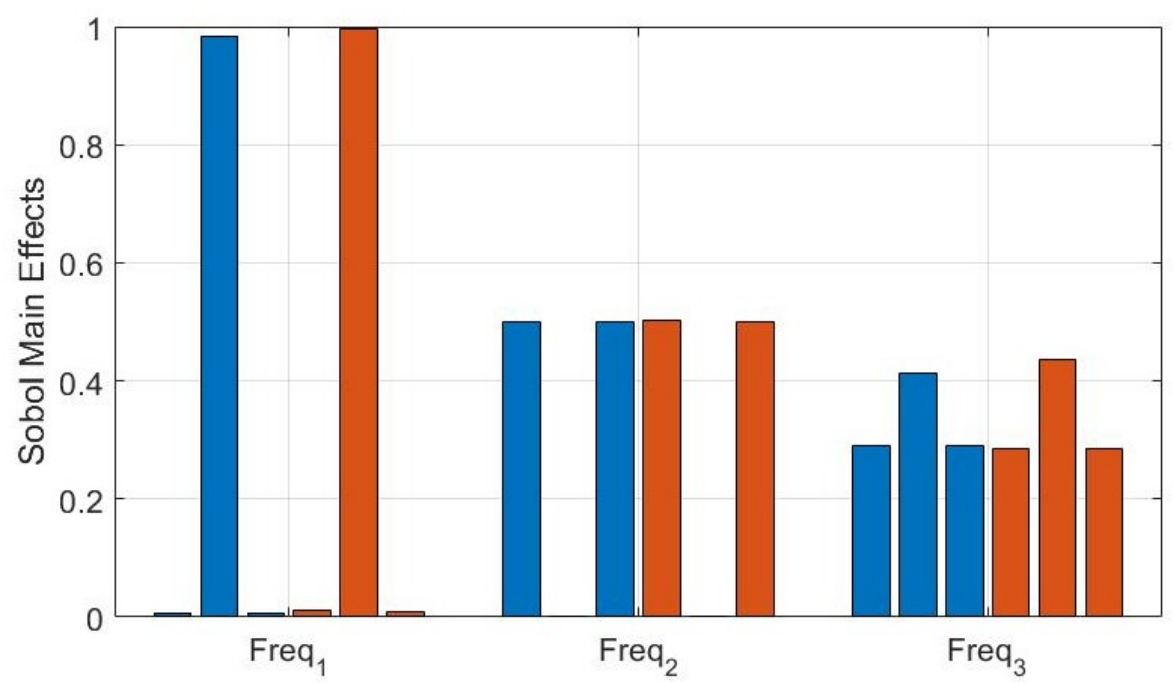

Figure 2.10: Comparison between the main effects for the first three frequencies. The first group (blue bars) takes in consideration the $S_{i}$ calculated analytically. The second group (orange bars) takes in consideration the $S_{i}$ estimated by LDD. The differences are negligible but the first are estimated by using directly the 8 test results, while LDD requires a much higher number of results, even in case of categorical variables. In this case $N_{s}=2048$ sample points are used. 
Three approaches are used in this chapter to estimate Sobol's sensitivities: the first one is based on an explicit polynomial regression which is used to derive analytical expression of Sobol's indices. The second approach uses the coefficient of determination in regression to make an estimation of the sensitivities by performing a partial linear regression which considers only a subset of parameters. This reformulation of Sobol's indices provides good results and allows to gain understanding on their meaning and use. The last approach considers the re-sampling methodology and the convergence of the indices is studied vs. the size of the sample. Even in case of categorical variable the needed sample size is much higher than the 8 simulation points used in the first two approaches. It is evident that in case of more complex models, where continuous variables define the uncertainty inputs, to create a reliable surrogate becomes a need. In the next chapter a detailed study about the generation of well-designed metamodels is presented. 


\section{Chapter 3}

\section{Meta-models}

\subsection{Introduction}

The construction of a meta-model, or surrogate model, takes a point of view that considers any numerical model to be a black box that produces a set of outputs based on a given set of inputs and model parameters. The main task of meta-models is to employ a scattered data fitting approach to replace such computer models with one that is much cheaper to evaluate while producing similar outputs when the input variables are restricted to a known domain.

Evidently, the construction of any meta-model requires a "computer design" whose main objective is sampling the deterministic numerical model efficiently. That implies dealing with the curse of dimensionality: the design should be able to effectively fill a high-dimensional space and to ensure an accurate solution of the scattered data fitting problem (see section 1.2.2). The way the fitting or approximation problem is solved (i.e. the number of points, the degree or the shape of the basis functions, the additional constraints, etc.) affects the meta-model's performance. This can be expressed in terms of efficiency and predictiveness. The process of evaluating this performance is known as model assessment, whereas the method for selecting the proper level of flexibility, to ensure the model's predictiveness, is known as model selection. Details on the model selection criteria can be found in many general books on statistics (e.g., [58, chapter 7]).

\subsubsection{Outline of the chapter}

Section 3.2 summarizes the least squares method Least Square Method (LSM) as a reliable tool to find the coefficients of the data approximation problem. The 
performance, which is expected to make a meta-model suitable for UQ applications, is defined and expressed in terms of efficiency and predictiveness. Then, the means for the performance's assessment are described, putting emphasis on the concepts of over-fitting and numerical stability.

Section 3.3 reviews radial kernels. The concept of anisotropic metric is further discussed, since it is key for the novel approach described in this research. While this class of kernels are well known in other fields, their use in the context of UQ and GSA has not been explored before, to the author' best knowledge. Remarkably, the addition of anisotropy in the design of surrogates is instrumental in improving their efficiency and predictiveness. In particular, this qualitative connection (see Forrester [24]) between "active dimensions" and relevant variables is studied and quantified by defining a link between the anisotropic metric and Sobol's sensitivities.

As an example of the usefulness of well-designed meta-models, an analytical function, the well-known Sobol's $G$-function, and two mechanical problems are studied in section 3.4 by means of surrogates. As will be verified, meta-models can provide an extremely robust and cost-effective tool for studying the sensitivity of complex systems and their stochastic response.

Finally, section 3.5 summarises the main conclusions and results of this research and proposes some possible extensions.

\subsection{Scatter data approximation by the least square method}

To introduce the notation that will be employed later, let us briefly describe next the standard least square approximation problem: if a model is sampled at $N_{s}$ points of the high-dimensional space $\mathcal{S} \equiv \mathbb{R}^{N_{p}}$, the generic output $Y_{n}=Y\left(X_{n}\right)$ evaluated at the input point $X_{n}=\left[X_{n 1}, X_{n 2}, \ldots, X_{n N p}\right]$, with $n \in\left[1,2, \ldots, N_{s}\right]$ and $N_{p}$ the total number of input parameters considered, can be approximated by a linear combination of $N_{k}$ basis or kernel functions $h_{k}: \mathcal{S} \rightarrow \mathbb{R}$ as

$$
Y\left(X_{n}\right) \simeq \sum_{k=1}^{N_{k}} \beta_{k} h_{k}\left(X_{n}\right) .
$$

This relation can be expressed more compactly using matrix notation as in

$$
Y(X) \simeq H(X) \beta
$$


where $H$ is the approximation or kernel matrix, $Y$ is the matrix of the $N_{\text {out }}$ QoIs evaluated at $N_{s}$ sampled points, $X$ is the sample matrix and $\beta$ is the vector of weights $\beta_{k}$ in the linear combination 3.2.

If the basis functions change during the 'learning' process, then the model is nonlinear and requires some numerical optimization to define the weights. This is computationally expensive, whereas keeping the model linear allows easier analysis and quicker computations.

Figure 3.1 explains the principle behind the construction of a meta-model: a sample matrix, which depends on the computer design and on the probabilities prescribed on the input variables, is created and an initial cloud of input-output pair of points is generated through the simulation model. This initial set is usually split into a training set, where the coefficients of regression are estimated and an evaluation set, which represents the unseen set of data used to estimate the error.

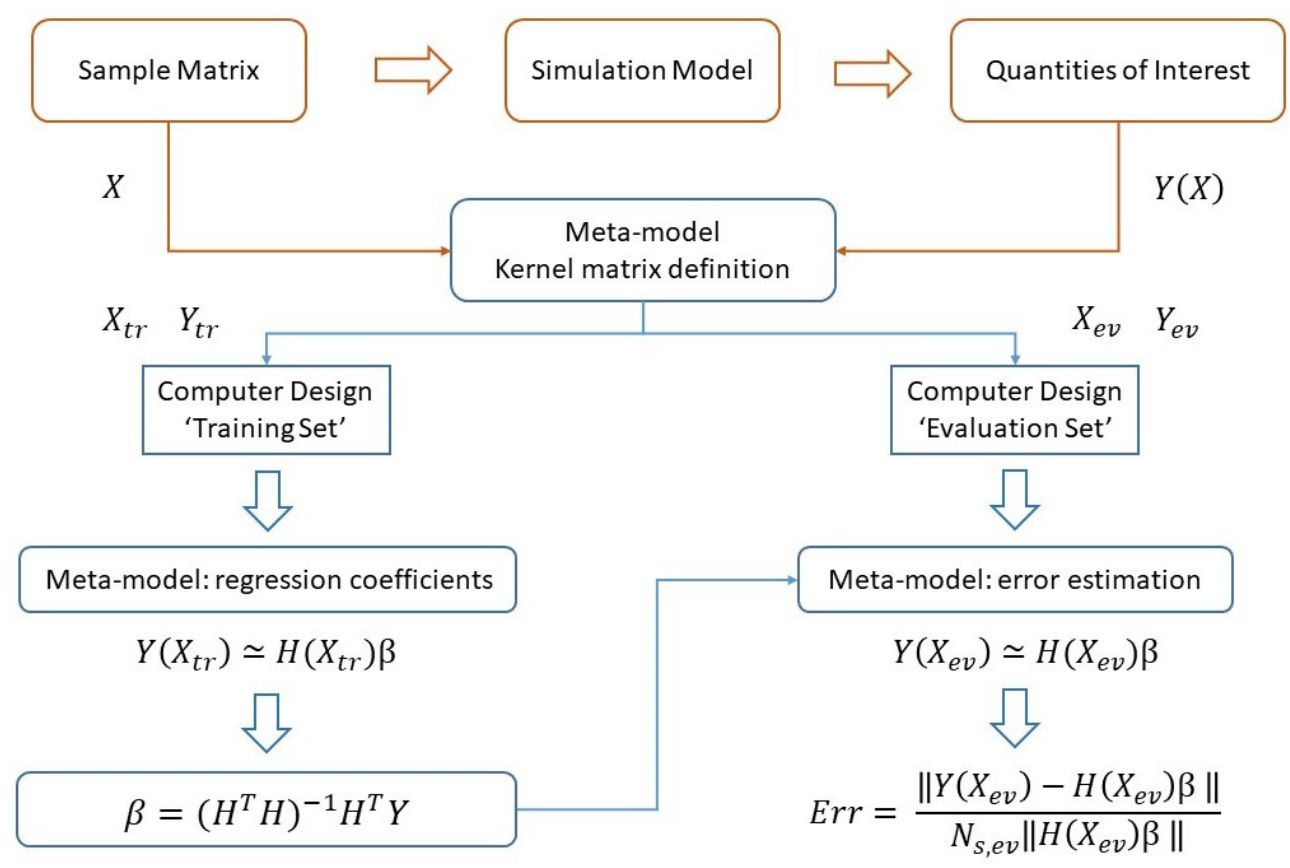

Figure 3.1: Meta-model construction: a sample matrix is considered and the QoIs evaluated through the simulation model. Then, this set is split in a usually wider training set where the coefficients of regression are estimated and in a smaller evaluation set, the unseen set of data, where the error can be evaluated.

Once the basis functions have been defined, the kernel matrix will depend only on the set while the coefficients of the expansion are considered indepen- 
dently from it. In this way, the regression coefficients are evaluated once for all on the training set and can be obtained minimising the sum of the squares of the residuals, i.e., as the minimisers

$$
\beta=\arg \min _{\beta^{*} \in \mathbb{R}^{N_{k}}}\left\|H \beta^{*}-Y\right\|^{2}
$$

The least square fitting on the training set has a closed-form solution:

$$
\beta=\left(H^{T} H\right)^{-1} H^{T} Y,
$$

where $H$ is assumed to have full column rank. Next, the error of the model can be estimated on an unseen set of data by evaluating its predictions on points of this set as

$$
Y\left(X_{e v}\right)=H\left(X_{e v}\right) \beta .
$$

Finally, the error on the evaluation set is estimated according to the standard expression:

$$
E r r=\frac{\left\|Y\left(X_{e v}\right)-H\left(X_{e v}\right) \beta\right\|}{N_{s, e v}\left\|H\left(X_{e v}\right) \beta\right\|}
$$

\subsubsection{Meta-model performance: efficiency and predictiveness}

In the context of UQ and GSA where the meta-model created on a coarse set of data will be then employed on much finer sets, two aspect have to be carefully considered: efficiency and predictiveness. In general, the number of simulation points required to achieve an acceptable fitting depends on the complexity of the model, the number of input parameters $N_{p}$, and their range of variability within the prescribed distributions. On the other hand, one has to consider that the use of meta-models makes sense when for some reason only a limited set of simulations is available. The most evident one is cost, but other scenarios can happen: for example not having access to the original model to run further analyses or having limited access to the license of the simulation program. The result is that the meta-model needs to be efficient: it should provide the best possible accuracy (lowest approximation error) when built from an available set of simulation data.

Figure 3.2 illustrates that depending on the number of simulations $N_{s}$ and the number of basis functions $N_{k}$ of the kernel matrix, the least square solution can be an interpolation. In this case, estimating the error on the training set does not make any sense because the meta-model is fully constrained to fit the 
output points. Otherwise, the unbiased estimate of the variance is:

$$
\sigma^{2}=\frac{\|Y-H \beta\|^{2}}{N_{s}-N_{k}} .
$$

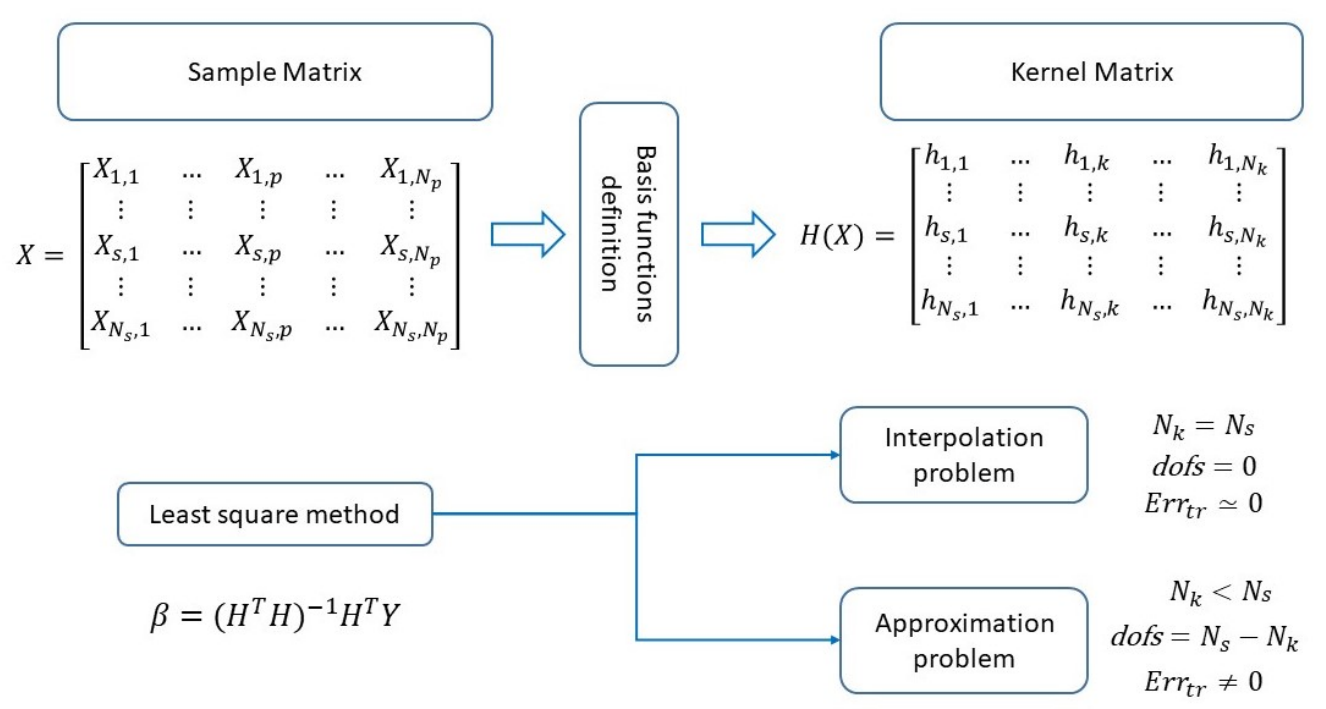

Figure 3.2: Meta-model approximation: depending on the number of basis functions $N_{k}$ in relation to the number of simulations points $N_{s}$, the least square solution can be an interpolation $\left(N_{k}=N_{s}\right)$ or an approximation $\left(N_{k}<N_{s}\right)$.

In statistics and machine learning this problem is known as the bias-variance dilemma: the model has to capture accurately the regularities in training data, but also generalizes well the unseen data. The meta-model needs to be predictive, also referred to as having generalization ability.

A simple, but effective way, to assess this ability is called out-of-sample validation, which consists in reserving from the available set of data a subset exclusively for the validation of the model (usually about $25 \%$ of it). It is the approach followed in this thesis and it was mentioned in Section 3.2.

If the error on the evaluation set is similar to the error obtained on the training set, the model can be considered to have good generalization ability. However, when the error on the training set is much smaller than the one on the evaluation set, the model suffers over-fitting, i.e., it is too sensitive to changes in the data. A possible solution, widely used in literature, is based on regularization techniques, for example Tikhonov's (some more details are given in Section 3.3). Finally, through the regularization parameter, some degrees of freedom are added to the scatter data approximation problem, helping to achieve numerical stability 
and avoiding over-fitting.

A second approach to avoid over-fitting consists in restricting the model to its simplest possible form, that is, limiting the dimension of the approximating space or, equivalently, the number of columns in the kernel matrix. In this thesis I rely on this second approach. It will be seen in the following sections, in the context of radial kernels, that a proper tuning of the basis functions can be very important in achieving a good meta-model, one that is able to cope with both performance aspects, efficiency and predictiveness.

\subsubsection{Considerations on meta-model assessment and selection}

It has to be considered that the assessment of the model can change as one makes a different choice for the points belonging to the training and evaluation sets. A better method which is intended to avoid the possible bias introduced by relying on any particular division is cross-validation. The available data set is partitioned in $q$ subsets, called folds. For the $k^{t h}$ fold, $k=1,2, \ldots, q$, the model is trained on the $q-1$ parts of the data and evaluated on the remaining part. Finally, one comes up with $q$ models, hopefully presenting similar performance. In this case, the same behavior can be expected on new unseen data.

In the cross-validation, the stability of the meta-model coefficients can also be checked, which further enhances the confidence in the regression. The error can be calculated as an average over the different partitions. An extreme version of this procedure is called Leave-One-Out Cross Validation (LOOCV), in which case $q=N_{s}$. Hence, the model is trained $N_{s}$ times on all the available data except for the $i^{t h}$ data point, which is the only one used for the evaluation of the error. In any case, the calculation of the average error for linear regression models does not require to solve the fitting problem $N_{s}$ times. In fact, Rippa [59] shows that the computation of the error due to $k^{\text {th }}$ leave-out point can be simplified to:

$$
\operatorname{Err}_{k}=\frac{\beta_{k}}{\left[\operatorname{diag}\left(H^{-1}\right)\right]_{k k}}
$$

where $\beta_{k}$ is the $k^{\text {th }}$ coefficient of the fitting problem solved on the total data set and $\left[\operatorname{diag}\left(H^{-1}\right)\right]_{k k}$ is the $k^{\text {th }}$ diagonal element of the inverse of the global kernel matrix. Since both terms need to be computed only once, the only additional operation required is averaging the LOOCV error over the full set, which 
is computationally inexpensive and given by the expresion

$$
\mathrm{LOOCV}=\frac{1}{N_{s}} \sum_{k=1}^{N_{s}} \operatorname{Err}_{k} .
$$

This remarkable result allows to obtain the LOOCV selection criterion fitting the model just once on all available observations.

The LOOCV is just an example because several selection criteria exist. Most of them make an adjustment to the mean squared error over the training set. Very popular are Akaike's Information Criterion (AIC) and the Schwarz Bayesian Information Criterion (BIC), both trying to minimize the loss of information which concerns the underlying unknown model. A good review of cross-validation is presented in [60], and a comprehensive recent survey can be found in [61]. Further details can be found in books on statistics (see, e.g., [58, chapter 7]).

Within this thesis I make use of the so-called Generalized Cross-Validation (GCV). It has been demonstrated to be a reliable and convenient model selection criteria since 1979 by Golub [62], particularly in the context of regularized least squares. Also Orr [63] emphasizes its use and compares the GCV against others model selection criteria. A revisited formulation of the GCV is given in appendix A.

\subsection{Meta-models based on radial kernels}

In this section the efficiency and predictiveness of a meta-model based on radial kernels are discussed. This type of kernels are functions of the form

$$
K(x, z): \mathbb{R}^{d} \times \mathbb{R}^{d} \longrightarrow \mathbb{R}^{+} \cup\{0\},
$$

with $K(x, z)=k(r(x, z))$, where $k: \mathbb{R}^{+} \cup\{0\} \longrightarrow \mathbb{R}^{+} \cup\{0\}$ is a continuous, monotonically decreasing function, invariant under both translations and rotations. The argument of $k$ is the Euclidean distance between points $x$ and $z$ in $\mathbb{R}^{d}$, that is:

$$
r(x, z)=\|x-z\|
$$

Radial kernels are often referred to as RBFs. To construct them, it is convenient to calculate the so-called Distance Matrix (DM) with components $r_{s, k}=\left|x_{s}-z_{k}\right|$, where $z_{k}$ are the centers of the radial functions and $x_{j}$ are the evaluation points of the latter. Hence, the DM has dimensions $N_{s} \times N_{k}$, where $N_{s}$ is the size of the sample and $N_{k}$ is the number of centers, and usually $N_{k} \leq N_{s}$. One of the 
benefits of considering RBFs for scattered data fitting is their natural extension to high dimensions.

\subsubsection{Shape parameter and anisotropic metrics}

A radial kernel makes use of the DM, but not only. Most of radial kernels' performance is affected by a shape parameter $\varepsilon$, which defines how localized the influence of the RBFs is. For example, in the case of the Gaussian radial kernel:

$$
K(x, z)=e^{-\varepsilon^{2} r(x, z)^{2}},
$$

a smaller value of the shape parameter makes the function flatter, while a larger one makes the function more peaked and localizes its influence.

Generally speaking, for a fixed number of centers, flatter kernels perform better in terms of accuracy of the interpolation problem until a certain threshold of the shape parameter, where the problem becomes numerically unstable. The same conclusions can be achieved if a fixed value of the shape parameter is considered and the number of centers is increased. In fact, in this case, the conditioning of the kernel matrix deteriorates as the minimum separation distance between centers, $h=\min \left\|z_{i}-z_{j}\right\|, i \neq j$, decreases.

In the last decade, mathematicians have approached the problem of finding better bases, in other words, approximation functions that can perform adequately at flat limits without incurring in ill-conditioning. Their construction typically involves numerical regularization, which employs additional information about the desired solution and serves to stabilise it [62, 64, 63, 65].

In any case, regularization techniques introduce at least one new parameter. For example, Tikhonov regularization, known in the context of statistics as ridge regression, defines the regularized solution as the Pareto optimum that combines the interpolation residuals and the 2 -norm of the regression coefficients $\beta$, as:

$$
\beta_{\lambda}=\arg \min _{\beta^{*} \in \mathbb{R}^{N_{k}}}\left\{\left\|H \beta^{*}-Y\right\|^{2}+\lambda\left\|\beta^{*}\right\|^{2}\right\},
$$

where, in addition to the symbols already defined in Eq. (3.2), the regularization parameter $\lambda$ is now introduced.

The regularization parameter $\lambda$ has to be optimized taking into consideration that the meta-model performance is very sensitive to the shape parameter $\varepsilon$.

One possible way for choosing the right $\lambda$ is linking the regularization parameter with the lowest prediction error as expressed by the GCV. An efficient algorithm for that is proposed in appendix A. 
In any case, usually, first a trial-and-error approach is used to define the shape parameter that minimizes the error on the unknown set of data and then, if it falls in a numerically unstable zone, some regularization strategy is employed. Some examples can be found in [31, chapter 2]. The procedure, depending to the complexity of the problem, can be cumbersome; additionally, it complicates the meta-model and increases the number of deterministic simulations required to build it.

Some authors have experimented with spatially varying shape parameters, which means assigning to each center a different scaling. This topic is particularly important in the context of Compactly Supported Radial Basis function, where a satisfactory interpolation is desired while preserving the sparsity of the kernel matrix. Radial kernels with different scales are often used by practitioners, but Fasshauer [31] and McCourt [32] highlight that, in this case, the interpolant is no longer generated by a single kernel and the theoretical foundations of the approximation must be reconsidered. In any case, the kernels are still isotropic, i.e. having different radius but each of them the same in all directions.

The same authors motivate the investigation of a coordinate-dependent scaling, leading to anisotropic radial kernels. In general, this type of kernels have not received much attention in the literature on approximation theory (see some exceptions by Casciola and co-workers in $[50,51])$. These works investigate the use of an anisotropic metric in the context of surface reconstruction and the regularizing properties of anisotropic kernels. Moreover, Fasshauer and McCourt [32] provide an interesting theoretical motivation for their potential use. A very brief review of their conclusions follows.

Fasshauer in [31, chapters 15 and 17], following the work of Wendland [66], discusses theoretically the estimation of the error approximation for strictly positive definite radial kernels. The error for kernel-based interpolation depends on the choice of the kernel, the location and the number $N_{k}$ of centers (i.e. the filling distance $h$ ), the sample size $N_{s}$, and the smoothness of the function to be approximated. Since for $N_{s}$ quasi-uniformly distributed data sites on a $d$-dimensional space the filling distance $h \propto N_{s}^{-1 / d}$, the rate of convergence deteriorates as $d$ increases. Hence, Fasshauer and McCourt in [32, chapter 9], discuss the opportunity to mitigate or even ensure a dimension-independent convergence rate allowing an anisotropic scaling of the kernels.

Within this research I consider metric parameters by using a diagonal, positive definite matrix $\Gamma=\operatorname{diag}\left(\gamma_{1}^{2}, \gamma_{2}^{2}, \ldots ., \gamma_{d}^{2}\right)$. Hence for example, an anisotropic 
Gaussian kernel is of the form:

$$
K(x, z)=e^{-\gamma_{1}^{2}\left(x_{1}-z_{1}\right)^{2}-\ldots-\gamma_{d}^{2}\left(x_{d}-z_{d}\right)^{2}}=e^{-(x-z)^{T} \Gamma(x-z)} .
$$

Figure 3.3 illustrates how an anisotropic kernel can be obtained. It will be function of the anisotropic metrics $\gamma=\left[\begin{array}{llllll}\gamma_{1} & \ldots & \gamma_{p} & \ldots & \gamma_{N_{p}}\end{array}\right]$ assigned to each direction. Further the generic term of the DM, $r_{s, k}(\gamma)$, can be affected by the shape factor $\varepsilon$, which leads to a kernel that depends both on the anisotropic metric and the shape parameter. The interaction between these two types of parameters will be studied next in Section 3.4.

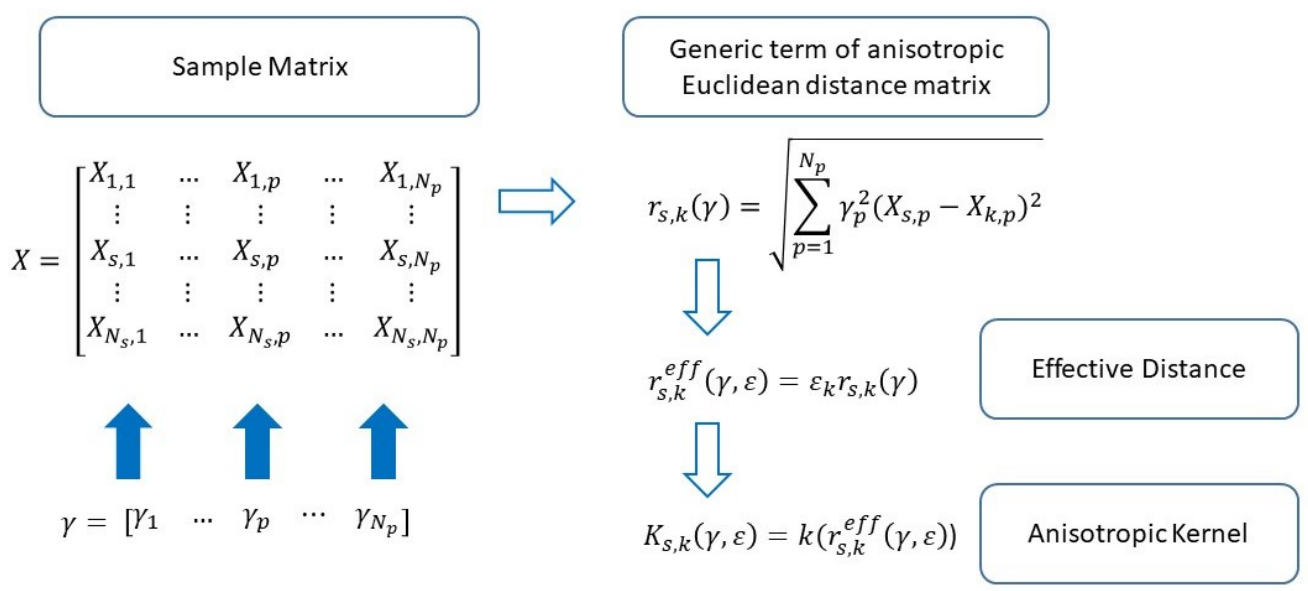

Figure 3.3: Anisotropic kernel matrix: computationally, the generic anisotropic kernel $K_{s, k}$ can be obtained multiplying each column of the sample by the corresponding anisotropic metric $\gamma_{p}$.

From a pragmatic point of view, having different scales for the parameters under consideration also makes sense because in most engineering applications the response of the system is dominated by a few dozens variables. An aspect to consider from a computational point of view is that optimizing, say, dozens of metric parameters, is not as prohibitive as optimizing hundreds of spatially varying shape parameters.

\subsubsection{Sobol LDD, GSA and anisotropic metrics}

Fassauher and McCourt in [32, section 16.3], conclude from their numerical experiments that "...enforcing anisotropy is most effective when the correct dimensions are emphasized." In general agreement with their observation, a strategy is 
planned to identify these correct dimensions and hence to be able to provide the optimization algorithm with some reasonable initial guess.

Saltelli at al. [21, section 2.4.7], suggests that a strategy to get the best possible results for a given Sobol set $N_{s}$, is defining $N_{s}$ on base- 2 and sorting the variable in the sample matrix according their relevance (first the most relevant). Very few knowledge exists a priori for that and consequently this last strategy requires at least one iteration in order to get the value of the global sensitivities and sort consequently the sample matrix. Additionally, assuming that the correct dimensions are those defined by the influential parameters, the sensitivity indices could be used to create a good initial guess for the optimized anisotropic metrics. For example, one can think to multiply the total orders by a constant factor. This approach does not guarantee to reach a global minimum but, at least, sets a systematic and unbiased method to achieve an optimal $\Gamma$.

\subsubsection{Detailed methodology}

Finally, let us summarize the main steps of the proposed methodology:

(1) Define the uncertainty variables and their probability distribution.

(2) Define the QoIs.

(3) Generate a cloud of outputs by sampling the deterministic model according to Sobol's LDD design. Previous knowledge or judgement can be used to sort the variables, the most influential the first, in order to get maximum benefits from Sobol's design (see Section 3.3.2).

(4) Split the input-output cloud of points in a training set and in an evaluation set (approximately $20 \%$ of the total one).

(5) Generate a meta-model based on anisotropic radial kernels according to the scheme presented in Figure 3.3.

(6) Find an optimal $\Gamma$ by minimising the error (3.6) on the evaluation set and by employing the classical LSM (Figure 3.2). The initial value of the matrix $\Gamma$ can be selected randomly.

(7) Find the global sensitivities according to the algorithm proposed by Saltelli [21, chapter 4]

(8) Rank the variables according to their relevance. If one wants to improve the meta-model, according to what is suggested in Section 3.3.2, two alternative routes are proposed: 
(a) Reduce the size of the problem by setting the non-influential variables, if any, to their mean value. Then, go back to step (3) by considering fewer variables and sorted according to their influence on the QoIs. Use the global sensitivities to define an initial guess for the metric $\Gamma$, as required at step (6).

(b) Return to step (6) and assign an initial value for $\Gamma$, setting each $\gamma_{i}$ equal to the corresponding global sensitivity scaled by some factor (i.e. 10). By doing that, the most relevant dimensions are favored.

(9) Iterate until a satisfactory meta-model is generated. According to my experience, two steps are enough in most cases.

\subsection{Applications}

In this section, an analytical function and two engineering problems are used for testing the performance of radial kernels based on optimised anisotropic metrics. Each of the examples focuses on a different aspect of the proposed methodology, as detailed next.

The first example considers Sobol's $G$-function, which is widely used in the literature on UQ methods [67]. As discussed by Saltelli [21] and Marrel [68], this function is used for various numerical estimation methods, including sensitivity analysis, because it is fairly complex, nonlinear, and non-monotonic. In addition, its sensitivity indices can be expressed in closed form.

The second analysis employs a physical model called the piston simulation function, used in [32] as a testing function for surrogates. The authors experiment with anisotropy and conclude that using the correct dimensions improves the approximation error. The specific choice of dimensions is, however, left unspecified. Following the analysis of Section 3.3.2, I study how the global sensitivity indices can be used to improve the meta-model performance. While it is obvious that they help in selecting the subset of influential parameters, and thus reducing the number of dimensions in the surrogate, I show they provide further information. In particular, they are useful in generating a sorted Sobol's design and providing a good initial guess for the anisotropic metrics.

The third example studies Taylor's impact problem. This is a very wellknown test $[69,70,71,72,73]$ used to study the rate dependent behavior of metallic materials at high strain rates and temperatures. Since computational models of such a test are expensive, a surrogate is a convenient way to study the propagation of uncertainties through the model. To study this problem, the 
commonly employed Johnson-Cook's material model is selected. Employing the meta-model, the global sensitivities of the anvil's length and diameter after the impact are calculated as functions of the five Johnson-Cook's model parameters and the impact's velocity. In this example I experiment with both the shape parameter and the anisotropic metrics in order to understand their possible synergies.

For simplicity, the examples in this section employ the $C^{4}$ Matérn radial kernel, which has the expression:

$$
K(x, z)=\left(1+\varepsilon\|x-z\|+\frac{1}{3} \varepsilon^{2}\|x-z\|^{2}\right) e^{-\varepsilon\|x-z\|},
$$

where $\varepsilon$ is the shape parameter.

The error on the unseen data, i.e. the evaluation set, is estimated according to Eq. 3.6. In the following analyses, I experiment with different sizes of the training set and a fix evaluation set in order to assess the error changes vs. the size of the training set. This is done to demonstrate that when anisotropic functions are employed, the error on the evaluation set is less sensitive to the size of the training set.

Both choices, type of kernel and error's metric, are made congruently with the work proposed in [32, chapter 16] and further discussed in section 3.4.2.

Evidently, other kernels could be successfully employed. My experience shows, however, that the benefits that derive from optimal anisotropic weighting are fairly robust with respect to the kernel and error metric choice.

\subsubsection{Sobol's G-function}

Sobol's $G$-function has the expression:

$$
G(X)=\prod_{i=1}^{d} \frac{\left|4 X_{i}-2\right|+a_{i}}{1+a_{i}},
$$

where $X_{i}$ are independent and identically distributed random variables that, for the purpose of uncertainty quantification, are assumed to be uniformly distributed, i.e., $X_{i} \sim \mathcal{U}[0,1]$, for all $i=1, \ldots, d$. In Eq. (3.15) a coefficient $a_{i} \geq 0$ is chosen for every random variable. The smaller the value of $a_{i}$, the higher the importance of the variable $X_{i}$. The partial variances of the first order are given by

$$
V_{i}=\frac{1}{3\left(1+a_{i}\right)^{2}},
$$


and the total variance can be expressed as a function of the first-order terms as:

$$
V=-1+\prod_{i=1}^{d}\left(1+V_{i}\right) .
$$

Thus, the first order effects are obtained as the ratios between the partial variances and the total one. The higher order effects can be calculated in the same way considering that the higher variance terms are given by the product of the corresponding first order variance, i.e. $V_{i j}=V_{i} V_{j}$.

In the proposed study, a 15 dimensional $G$-function is considered. Three training sets are used of size $N_{t r}=2^{k}$ with $k=8,10$ and 12 , with a shape parameter ranging from 0.2 to 3 , with steps of size 0.1 , and the error (3.6) is evaluated on a fixed set of 100 unseen points. Also, the first order indices obtained from the meta-model are compared with their theoretical counterparts. For that, the values of the coefficients $a_{i}$ are selected according to the expression $a_{i}=$ $(i-1) \cdot 2^{i-4}$, and the $C^{4}$ Matérn kernel (3.14) is employed. For each index $i$, a lower value of $a_{i}$ indicates a higher sensitivity index and a greater relevance of the input variable $X_{i}$.

Fig. 3.4 shows the approximation error on the evaluation set vs. the value of the shape parameter in several training sets. The results without and with enforced anisotropy are represented, respectively, by continuous and discontinuous lines. As expected, in the case of ordinary LSM (continuous lines) and in absence of numerical singularities, the smaller the shape parameter, the smaller the error. Also, increasing the training set provides a better accuracy. On the other hand, when anisotropic metrics are employed (discontinuous lines) a smaller error is achieved for all values of the shape parameter. Additionally, the size of the training set has a smaller impact on the accuracy of the approximation.

Table 3.1 shows, for each random variable $X_{i}$ and each coefficient $a_{i}$, the analytical value of the first order sensitivities and its comparison with the value obtained by employing a meta-model based on anisotropic radial kernels. The analytical and numerical values are very close and reveals the relevance of the first four variables over the fifteen considered at the beginning. Estimating the sensitivity indices required about half a million simulations with the meta-model, a rather inexpensive computation.

This analytical example reveals some interesting properties of the anisotropic approximation functions. First, the approximation error appears to be almost insensitive to the shape parameter and very robust with respect to the size of the training set. Second, the accuracy in the prediction of the sensitivity indices 
highlights the accuracy of these meta-models over sets of unseen data.

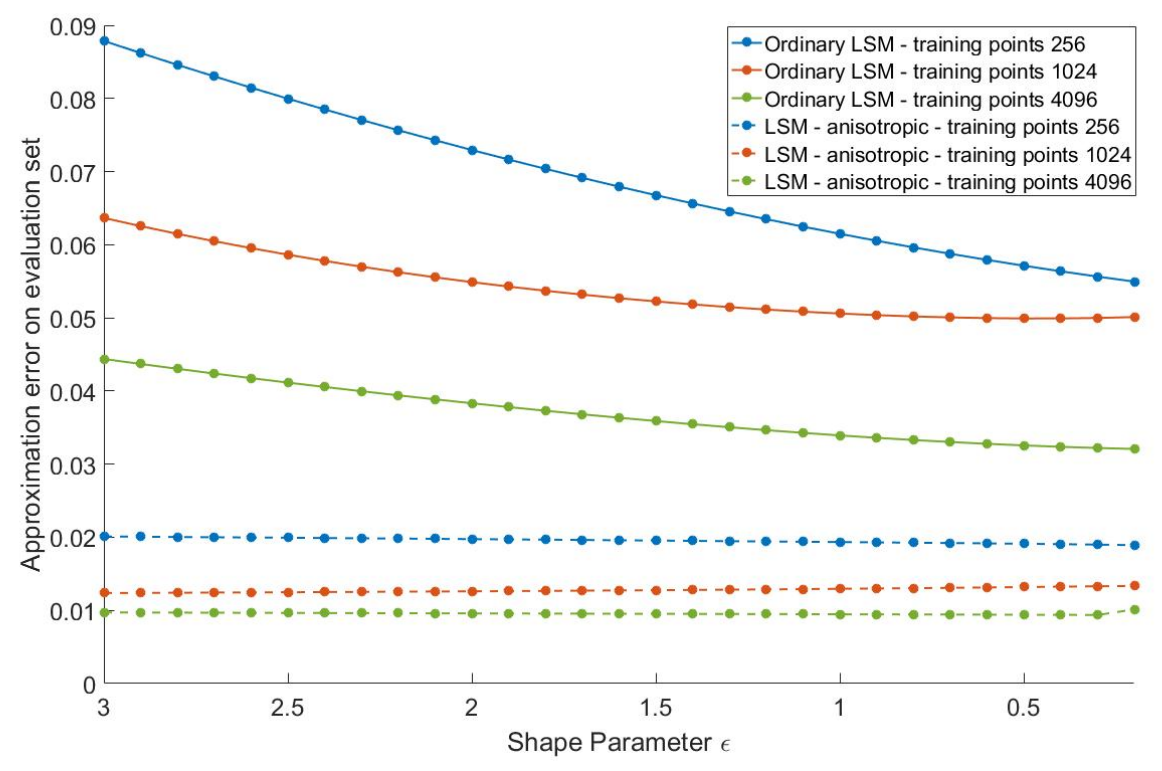

Figure 3.4: $G$-function in dimension 15 - error vs. shape parameter. The plot compares the solution obtained through the ordinary LSM (continuous lines) with those obtained with anisotropic metrics (discontinuous lines) with different sizes of the training set. In general, anisotropic metrics provide smaller errors that, furthermore, are almost independent of the shape parameter.

\subsubsection{Piston simulation function}

The piston function evaluates the time $C$, in seconds, required by the circular motion of a piston in a cylinder to complete one cycle. This function is used by Fasshauer and McCourt [32, chapter 16], for testing surrogate models. Now, the same analysis is considered with some slight variations. First, relying on Sobol's LDD instead of the Halton set for sampling the underlying model. Second, the advantages of employing anisotropic kernels with optimised metrics are studied. Finally, the sensitivity indices, of first and total orders, are found so as to rank the parameters according to their relevance.

The piston function is defined as:

$$
C(X)=2 \pi \sqrt{\frac{W}{k+S^{2} \frac{P_{0} V_{0}}{T_{0}} \frac{T_{a}}{V^{2}}},}
$$




\begin{tabular}{cccc}
\hline Random variable & Coefficient $a_{i}$ & $S_{i}$ (analytical) & $S_{i}$ (meta-model) \\
\hline$X_{1}$ & 0 & $41.76 \%$ & $42.64 \%$ \\
$X_{2}$ & 0.25 & $26.73 \%$ & $27.48 \%$ \\
$X_{3}$ & 1 & $10.44 \%$ & $10.00 \%$ \\
$X_{4}$ & 3 & $2.61 \%$ & $2.32 \%$ \\
$X_{5}$ & 8 & $0.52 \%$ & $0.42 \%$ \\
$X_{6}$ & 20 & $0.09 \%$ & $0.07 \%$ \\
$X_{7}$ & 48 & $0.02 \%$ & $0.03 \%$ \\
$X_{8}$ & 112 & $0.00 \%$ & $0.00 \%$ \\
$X_{9}$ & 256 & $0.00 \%$ & $0.00 \%$ \\
$X_{10}$ & 576 & $0.00 \%$ & $0.00 \%$ \\
$X_{11}$ & 1280 & $0.00 \%$ & $0.00 \%$ \\
$X_{12}$ & 2816 & $0.00 \%$ & $0.00 \%$ \\
$X_{13}$ & 6144 & $0.00 \%$ & $0.00 \%$ \\
$X_{14}$ & 13312 & $0.00 \%$ & $0.00 \%$ \\
$X_{15}$ & 28672 & $0.00 \%$ & $0.00 \%$ \\
\hline
\end{tabular}

Table 3.1: $G$-function in dimension 15 - First order sensitivity indices: comparison between analytical results and those obtained numerically by a meta-model with anisotropic kernel. The surrogate is constructed by employing a training set of $N_{t r}=1024$ points and a shape parameter $\varepsilon=3$.

where:

$$
V=\frac{S}{2 k}\left(\sqrt{A^{2}+4 k \frac{P_{0} V_{0}}{T_{0}} T_{a}}-A\right),
$$

and

$$
A=P_{0} S+19.62 W-\frac{k V_{0}}{S} .
$$

The input variables are collected in the random vector:

$$
X=\left[W, S, V_{0}, k, P_{0}, T_{a}, T_{0}\right] \in \mathbb{R}^{7} .
$$

Each of these variables is assumed to follow a uniform distribution. Table 3.2 reports the lower and upper bounds, together with the meaning and the units of each input parameter. It is a good practice to re-scale the variables' domain to the non-dimensional interval $[0,1]$ using a linear mapping. In the study, the first 500 points are used as evaluation set while the training set is increased from 100 to 10000 points. Three radial kernels are tested by the authors, but I just employ the $C^{4}$ Matérn surrogate with anisotropic metrics $\gamma=3^{m}$, with $m=$ $2,1.5,1, \ldots,-1$. When the largest training set is employed, the approximation error on the evaluation set becomes $E r r_{e v}=1.313 \times 10^{-4}$. Table 3.3 shows 
the metric used for each variable. The authors used a square kernel matrix, hence each point belonging to the training set is also a centre, $N_{s}=N_{k}$. The smallest error is obtained applying the least square method on a kernel matrix of dimensions $10000 \times 10000$.

\begin{tabular}{ccclr}
\hline Variable & Lower bound & Upper Bound & Meaning & Units \\
\hline$W$ & 30 & 60 & Piston weight & $\mathrm{kg}$ \\
$S$ & 0.005 & 0.020 & Piston surface area & $\mathrm{m}^{2}$ \\
$V_{0}$ & 0.002 & 0.010 & Initial gas volume & $\mathrm{m}^{3}$ \\
$k$ & 1000 & 5000 & Spring coefficient & $\mathrm{N} / \mathrm{m}$ \\
$P_{0}$ & 90000 & 110000 & Atmospheric pressure & $\mathrm{N} / \mathrm{m}^{2}$ \\
$T_{a}$ & 290 & 296 & Ambient temperature & $\mathrm{K}$ \\
$T_{0}$ & 340 & 360 & Filling gas temperature & $\mathrm{K}$ \\
\hline
\end{tabular}

Table 3.2: Piston function - random variables.

\begin{tabular}{cccccccc}
\hline Variables & $W$ & $S$ & $V_{0}$ & $k$ & $P_{0}$ & $T_{a}$ & $T_{0}$ \\
\hline$\gamma$ & 9.0000 & 5.1962 & 3.0000 & 1.7321 & 1.0000 & 0.5774 & 0.3333 \\
\hline
\end{tabular}

Table 3.3: Piston function - anisotropic metrics from [32, chapter 16].

The anisotropic scaling is obtained applying the $N_{p}$ anisotropic metrics during the process of evaluating the distance matrix (see Figure 3.3 for details). In particular, the factor corresponding to any input variable multiplies the corresponding column of the sample matrix.

\section{Exploiting Sobol' design.}

Table 3.4 reports the error on a fixed evaluation set as the points in the training set change. In the second column the results are those presented by Fasshauer and McCourt. The third column presents the results when the Sobol' LDD is used instead of the Halton LDD: the error is almost the same for smaller sets but improves for larger ones. The difference obtained in the error is calculated as:

$$
\Delta E r r=\frac{E r_{e v, \text { Halton }}-E_{\text {rr }} r_{\text {evobol }}}{E r r_{e v, \text { Halton }}} \times 100[\%] .
$$

A positive $\Delta E r r$ represents an improvement with respect the error estimated by the Halton set, while a negative one indicates an increment of the initial error. In 
the last case, $N_{s}=10000$, just changing the computer design implies improving the error on the evaluation set about $37 \%$.

Let us discuss the results presented in the fifth column which correspond to a sorted Sobol' design and whose improvement is significant in spite of the size of the training set. For example, in the last case the improvement increases from $37.3 \%$ (see table 3.4 ) to $68 \%$. In other words, the error obtained with an Halton set of 10000 points is roughly the same obtained with the sorted Sobol' design with only 3593 points.

Saltelli at al. [21, section 2.4.7], suggests that a strategy to get the best possible results for a given Sobol' set $N_{s}$, is defining $N_{s}$ on base- 2 and sorting the variable in the sample matrix according their relevance (first the most relevant). Very few knowledge exists a priori for that and consequently this last strategy requires at least one iteration. This sorting effect seems to have not received much attention in the literature, but from my experiments I conclude that it is well worth the effort. The variables presented in Table 3.3 are re-ordered according to this criterion as:

$$
X^{*}=\left[S, V_{0}, W, k, P_{0}, T_{0}, T_{a}\right] \in \mathbb{R}^{7} .
$$

The piston weight $W$ is moved to the third position and $T_{0}$ now precedes $T_{a}$. Evidently, a first rough GSA iteration has to be performed, which allows to rank the variables according their influence. The ordering in the metric coefficients is kept unchanged, which implies that the more relevant the variable, the higher the associated anisotropic metric parameter is. This motivates the study presented in the next section, where I look at the benefits in optimizing the anisotropic metric.

\section{Exploiting the anisotropy.}

Using Sobol's design with some fine-tuning helps considerably in getting more efficiency and predictiveness. Additionally, I try now to optimize the anisotropic metric and evaluate if the meta-model performance can be further improved. The evaluation set is unchanged, while for the training set a Sobol' LDD of $N_{s}=2^{m}$, with $m=10,11,12,13$, points is employed. The sizes of the training set are slightly different from those ones previously used because I want to get the maximum benefit from Sobol's design using a base- 2 sequence. The variables are kept sorted, according to the studies presented in section 3.4.2.

The results are presented in Table 3.5: the meta-model's performance is 


\begin{tabular}{lccrcr}
\hline Training set & $\begin{array}{c}\text { Halton LDD } \\
N_{s}\end{array}$ & $\begin{array}{c}\text { Sobol' LDD } \\
\text { Matérn C4 }\end{array}$ & Matérn C4 & \multicolumn{3}{c}{$\begin{array}{c}\text { Sorted Sobol' } \\
\text { Matérn C4 }\end{array}$} & $\Delta E r r$ \\
\hline 100 & $2.144 \times 10^{-3}$ & $2.306 \times 10^{-3}$ & $\mathbf{- 7 . 6 \%}$ & $1.782 \times 10^{-3}$ & $\mathbf{1 6 . 9 \%}$ \\
166 & $1.487 \times 10^{-3}$ & $1.843 \times 10^{-3}$ & $\mathbf{- 2 3 . 9 \%}$ & $1.421 \times 10^{-3}$ & $\mathbf{4 . 5 \%}$ \\
278 & $1.149 \times 10^{-3}$ & $1.142 \times 10^{-3}$ & $\mathbf{0 . 6 \%}$ & $9.021 \times 10^{-4}$ & $\mathbf{2 1 . 5 \%}$ \\
464 & $7.678 \times 10^{-4}$ & $9.603 \times 10^{-4}$ & $\mathbf{- 2 5 . 1 \%}$ & $5.894 \times 10^{-4}$ & $\mathbf{2 3 . 2 \%}$ \\
774 & $6.214 \times 10^{-4}$ & $6.602 \times 10^{-4}$ & $\mathbf{- 6 . 3 \%}$ & $4.019 \times 10^{-4}$ & $\mathbf{3 5 . 3 \%}$ \\
1291 & $4.310 \times 10^{-4}$ & $4.351 \times 10^{-4}$ & $\mathbf{- 0 . 9 \%}$ & $2.380 \times 10^{-4}$ & $\mathbf{4 4 . 8 \%}$ \\
2154 & $3.204 \times 10^{-4}$ & $3.086 \times 10^{-4}$ & $\mathbf{3 . 7 \%}$ & $1.633 \times 10^{-4}$ & $\mathbf{4 9 . 0 \%}$ \\
3593 & $2.940 \times 10^{-4}$ & $1.980 \times 10^{-4}$ & $\mathbf{3 2 . 7 \%}$ & $1.131 \times 10^{-4}$ & $\mathbf{6 1 . 5 \%}$ \\
5994 & $1.749 \times 10^{-4}$ & $1.457 \times 10^{-4}$ & $\mathbf{1 6 . 7 \%}$ & $6.961 \times 10^{-5}$ & $\mathbf{6 0 . 2 \%}$ \\
10000 & $1.313 \times 10^{-4}$ & $8.234 \times 10^{-5}$ & $\mathbf{3 7 . 3 \%}$ & $4.197 \times 10^{-5}$ & $\mathbf{6 8 . 0 \%}$ \\
\hline
\end{tabular}

Table 3.4: Piston function - Halton vs. Sobol' LDD.

further and significantly improved. In spite of the reduced size of the training set, the error is about one order of magnitude smaller and the improvement is roughly $80 \%$. These are very promising results: the optimised anisotropic metric compensates the smaller size of the sets keeping the approximation error on the evaluation set small. For example, the result obtained in Table 3.5 with an optimized metric and a set of 1024 points is very similar to the one obtained without optimization but with a larger set of 8192 points.

Therefore, the GSA can be considered as a tool not only for getting the best benefits from Sobol's design but also as a guideline for the initial guess of the anisotropic metric in the optimization routine. Extending my statement in Section 3.3.2, I can now conclude that “... enforcing anisotropy is most effective when the correct dimensions are emphasised. Moreover, GSA is a reliable tool to understand what the correct dimensions are."

\begin{tabular}{cccc}
\hline $\begin{array}{c}\text { Training set } \\
N_{s}\end{array}$ & $\begin{array}{c}\text { Sobol' LDD } \\
\text { Matern C4 }\end{array}$ & $\begin{array}{c}\text { Sobol' LDD with } \gamma_{\text {opt }} \\
\text { Matern C4 }\end{array}$ & $\Delta E r r$ \\
\hline 1024 & $2.829 \times 10^{-4}$ & $5.44 \times 10^{-5}$ & $\mathbf{8 0 . 8 \%}$ \\
2048 & $1.689 \times 10^{-4}$ & $3.29 \times 10^{-5}$ & $\mathbf{8 0 . 5 \%}$ \\
4096 & $1.108 \times 10^{-4}$ & $1.88 \times 10^{-5}$ & $\mathbf{8 3 . 0 \%}$ \\
8192 & $4.940 \times 10^{-5}$ & $9.07 \times 10^{-6}$ & $\mathbf{8 1 . 6 \%}$ \\
\hline
\end{tabular}

Table 3.5: Piston function - error evaluation with optimised anisotropic metric. 


\begin{tabular}{cccccccc}
\hline & $S$ & $V_{0}$ & $W$ & $k$ & $P_{0}$ & $T_{0}$ & $T_{a}$ \\
\hline w/o opt. & 9.0000 & 5.1962 & 3.0000 & 1.7321 & 1.0000 & 0.5774 & 0.3333 \\
1024 & 1.8282 & 1.0242 & 0.3061 & 1.3215 & 0.0775 & 0.0246 & 0.0173 \\
2048 & 1.7639 & 1.1278 & 0.3103 & 1.3684 & 0.0850 & 0.0439 & 0.0143 \\
4096 & 1.9243 & 1.1722 & 0.3487 & 1.2308 & 0.0849 & 0.0506 & 0.0117 \\
8192 & 2.7494 & 1.9968 & 0.4836 & 1.9020 & 0.1419 & 0.0960 & 0.0217 \\
\hline
\end{tabular}

Table 3.6: Piston function - optimised anisotropic metric.

\section{Global Sensitivity Analysis.}

In this last part of the analysis, the first order $S_{i}^{I}$ and the total sensitivities $S_{i}^{T}$ are estimated so as to select the input variables which affect the variance the most. Since no analytical expressions can be obtained in this model for Sobol's indices, the main and total effects reference values are evaluated using the exact piston function, without any meta-model. The results are then compared when the indices are evaluated by means of the radial kernel meta-model obtained in the previous section with a sample of $N_{s}=1024$ points and whose approximation error is shown in Table 3.5.

The evaluation of the sensitivity indices employs a sample matrix of $2^{16}$ points, and hence the model is evaluated $N_{\text {run }}=N_{s}\left(N_{p}+2\right)=9 \times 2^{16}$ times. Table 3.7 shows the comparison of the mean, the variance and the sensitivities indices obtained with the original model vs. those ones obtained with the meta-model, demonstrating the good accuracy of the latter. The largest relative errors correspond to the sensitivity indices of variables $T_{0}$ and $T_{a}$, which are, however, small in absolute terms.

Now the GSA results can be discussed. A convenient way to understand these concepts is to make use of pie charts of the first and total effects (see Figure 3.5). From them one deduces that the variables $T_{0}$ and $T_{a}$ do not have a significant contribution on the output variance and can conveniently be fixed at their mean value, while the first four variables affect the output variance the most. Particularly, the piston surface $S$ and the initial gas volume $V_{0}$, explain roughly the $90 \%$ of the variance in the output time.

It is worth noting that the output variance cannot be explained completely by the first effects, i.e. the sum of the first sensitivity indices is less than one. The unexplained effect is about $6 \%$ and is due to the interactions among the variables. In contrast, the sum of the total effects can be greater than one and the percentages showed in the pie chart are redistributed according to their sum. 
Finally, the GSA can be used as a dimensional reduction tool. In fact, in this study one can think to generate a more efficient meta-model by selecting only the relevant parameters. That would allow to use a smaller training set for the meta-model and a smaller set of simulations to estimate Sobol's indices.

\begin{tabular}{llccr}
\hline & & Original Model & Meta-model $\left(N_{s}=1024\right)$ & $\Delta E r r$ \\
\hline & Mean(Y) & 0.462 & 0.463 & $\mathbf{- 0 . 0 0 3 \%}$ \\
& V(Y) & 0.140 & 0.139 & $\mathbf{0 . 0 1 4 \%}$ \\
\hline$S$ & S1 & $5.57 \times 10^{-1}$ & $5.57 \times 10^{-1}$ & $\mathbf{- 0 . 0 5 6 \%}$ \\
$V_{0}$ & S2 & $3.21 \times 10^{-1}$ & $3.21 \times 10^{-1}$ & $\mathbf{0 . 0 7 9 \%}$ \\
$W$ & S3 & $3.91 \times 10^{-2}$ & $3.92 \times 10^{-2}$ & $\mathbf{- 0 . 2 5 6 \%}$ \\
$k$ & S4 & $2.06 \times 10^{-2}$ & $2.06 \times 10^{-2}$ & $\mathbf{- 0 . 2 8 1 \%}$ \\
$P_{0}$ & S5 & $1.24 \times 10^{-3}$ & $1.24 \times 10^{-3}$ & $\mathbf{- 0 . 0 2 9 \%}$ \\
$T_{0}$ & S6 & $2.40 \times 10^{-5}$ & $2.57 \times 10^{-5}$ & $\mathbf{- 7 . 1 0 4 \%}$ \\
$T_{a}$ & S7 & $5.04 \times 10^{-6}$ & $3.83 \times 10^{-6}$ & $\mathbf{2 3 . 8 8 8 \%}$ \\
\hline$S$ & ST1 & $5.99 \times 10^{-1}$ & $5.99 \times 10^{-1}$ & $\mathbf{- 0 . 0 3 6 \%}$ \\
$V_{0}$ & ST2 & $3.53 \times 10^{-1}$ & $3.52 \times 10^{-1}$ & $\mathbf{0 . 1 6 4 \%}$ \\
$W$ & ST3 & $5.09 \times 10^{-2}$ & $5.09 \times 10^{-2}$ & $\mathbf{- 0 . 1 3 1 \%}$ \\
$k$ & ST4 & $6.70 \times 10^{-2}$ & $6.68 \times 10^{-2}$ & $\mathbf{0 . 2 9 5 \%}$ \\
$P_{0}$ & ST5 & $1.35 \times 10^{-3}$ & $1.34 \times 10^{-3}$ & $\mathbf{0 . 3 2 3 \%}$ \\
$T_{0}$ & ST6 & $7.02 \times 10^{-5}$ & $6.93 \times 10^{-5}$ & $\mathbf{1 . 2 9 7 \%}$ \\
$T_{a}$ & ST7 & $9.02 \times 10^{-6}$ & $9.17 \times 10^{-6}$ & $\mathbf{- 1 . 7 0 3 \%}$ \\
\hline
\end{tabular}

Table 3.7: Piston function - First order and total sensitivity indices.

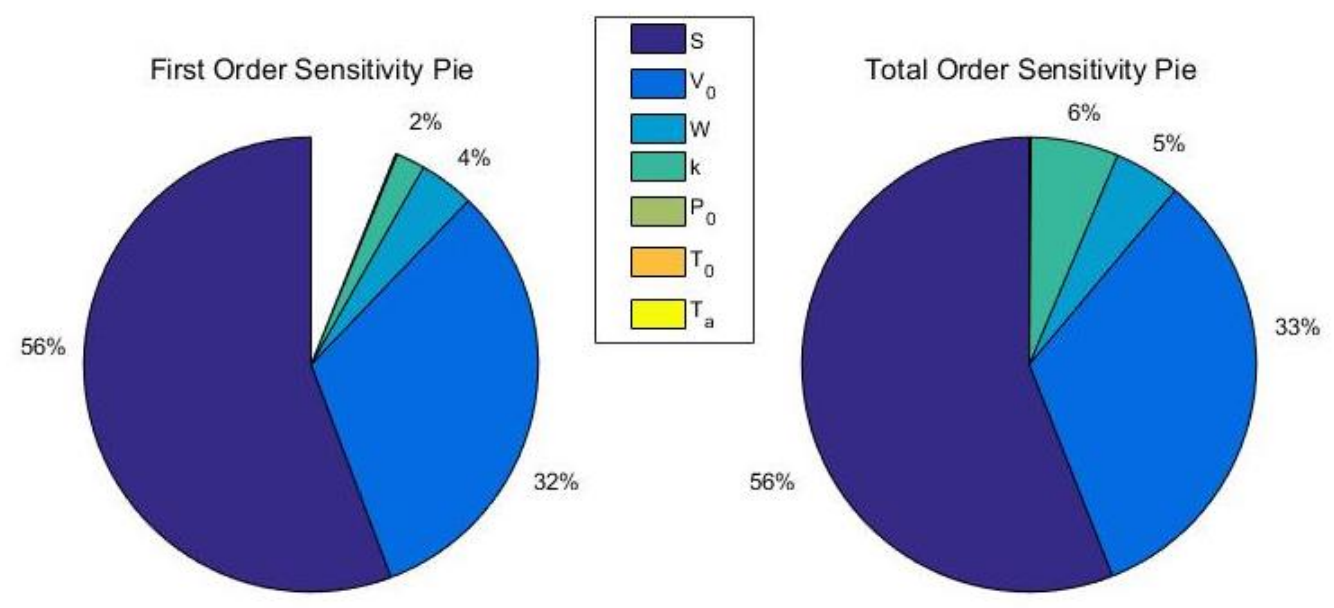

Figure 3.5: Piston function - GSA indices. 


\subsubsection{Taylor's impact test}

Taylor's anvil impact test is one of the most common procedures to study the rate dependency of the plastic deformation in metals $[69,70,71,72,73]$. In this test, a cylindrical specimen of length $L_{0}$ and diameter $\phi_{0}$ is launched against a rigid wall with a velocity $v$, permanently deforming it in a way that can be easily measured. For example, the deformed length and diameter at the impact section, denoted respectively as $L$ and $\phi$, are two dimensions that can be accurately measured and used to gain insight into the material behavior. See Figure 3.6 for an illustration.
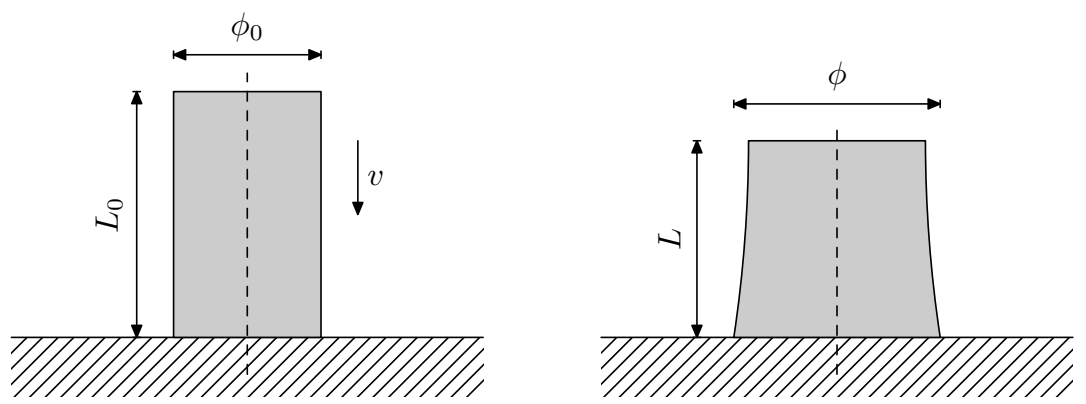

Figure 3.6: Schematic of anvil in Taylor's impact test.

The simplicity of Taylor's experiment has made it widely employed as benchmark for material models of rate dependent plasticity. Among these, one of the most widely employed is Johnson-Cook's model [74, 73]. This is a von Mises type plasticity model in which the yield stress depends on the accumulated plastic strain, its rate, and the temperature. More specifically, the yield stress of Johnson-Cook's model has the expression

$$
\sigma_{y}=\left(A+B \epsilon^{N}\right)\left(1+C \log \frac{\dot{\epsilon}}{\epsilon_{0}}\right)\left(1-\left(\frac{T-T_{0}}{T_{m}-T_{0}}\right)^{M}\right) .
$$

Here, $\epsilon$ is the accumulated plastic strain and $\dot{\epsilon}$ its time derivative, $T, T_{m}$ and $T_{0}$ are, respectively, the current, melting, and reference temperatures of the material. Finally, $A, B, N, M, \dot{\epsilon}_{0}$ are the five material parameters of the plasticity model. The complete material model requires, in addition, the elastic constants completely determined, for example, by Lamé parameters $\lambda$ and $\mu$.

I study next the UQ in a model of Taylor's test that is based on JohnsonCook's constitutive law. In this test, the input variables are the ten constants of the material model, the density $\rho$ of the material, and the impact velocity. More specifically, the impact of a copper cylinder is studied, as in the works 
$[70,75,76,71]$ with parameters

\begin{tabular}{ccccccc}
$A$ & $B$ & $C$ & $N$ & $M$ & $T_{0}$ & $T_{m}$ \\
$\mathrm{MPa}$ & $\mathrm{MPa}$ & - & - & - & $\mathrm{K}$ & $\mathrm{K}$ \\
\hline 90 & 292 & 0.025 & 0.31 & 1.09 & 300.0 & 1356.0
\end{tabular}

Table 3.8: Johnson-Cook constants for copper.

The Lamé elastic constants are $\lambda=150 \times 10^{3} \mathrm{MPa}, \mu=75 \times 10^{3} \mathrm{MPa}$, and the density $\rho=8960 \mathrm{~kg} / \mathrm{m}^{3}$. The tests are performed under isothermal conditions and $T=300 \mathrm{~K}$. The initial diameter and length of the cylinders are, respectively, $7.62 \mathrm{~mm}$ and $25.4 \mathrm{~mm}$.

To simulate the impacts a finite element model consisting of 1680 isoparametric hexahedra is employed, meshing one quarter of the rod. Johnson-Cook's model is implemented in the open source library MUESLI [77].

\section{Strategy for non-intrusive propagation of inputs uncertainty.}

My UQ process relies in a non-intrusive approach based on Sobol's design. Six stochastic inputs are considered: the five material constants $A, B, C, N, M$, whose nominal values are shown in Table 3.8, and the impact velocity, whose nominal value is $190 \mathrm{~m} / \mathrm{s}$. Two QoIs are considered, the increment of the diameter in the impacting surface and the anvil's length reduction, both measured $80 \mathrm{~ms}$ after the initiation of the contact.

Uniform probability density functions are prescribed on the five material constants with a scatter of $\pm 5 \%$ on their nominal values, and a normal distribution on the velocity, with mean equal to its nominal value and a standard deviation equal to $3 \%$ of its mean. To the author's knowledge, there exists no UQ analysis in the literature on Taylor's test, and the probability distributions on the input parameters are selected to the best of my engineering judgment.

Because of the nonlinear constitutive equations of the material and the large elasto-plastic deformations experienced by the anvils during the impact, Taylor's test simulation requires an expensive computational model which cannot be directly used in a sample-based UQ process. Thus, to allow the propagation of the input uncertainties and accordingly to estimate the global sensitivities of the QoIs, a reduced cloud of points is first used to generate a meta-model. Specifically, the $C^{4}$ Matérn kernel is employed and the advantages in considering anisotropic metrics are explored by using the approach described next. 


\section{Meta-model generation and selection.}

For this example the $C^{4}$ Matérn kernel (3.14) is used again. The anisotropic scaling is obtained applying the $N_{p}$ anisotropic metrics during the process of evaluating the distance matrix.

To have a robust approach to meta-model generation, the stochastic input variables are re-scaled, through a linear mapping, from their physical domain onto a hypercube $[0,1]^{d}$ with $d=6$, where all of them are normalized. To create the cloud of deterministic points, 306 simulations are run. Several $C^{4}$ Matérn kernel surrogates are created employing wider training sets, i.e., 32, 64, 128, 256 points, while the last 50 points are reserved to evaluate the error.

Since new parameters for the kernels are introduced, their effect in combination with the well-known shape parameter has to be analyzed. Smaller values of the shape factor lead to flatter kernels, which usually produce smaller approximation errors until a certain optimum value. One of the drawbacks of this approach is that when large training set are used, i.e., thousands of points, in combination with low value of shape parameter the minimum of the error can be found in an unstable region, where the kernel matrix becomes ill-conditioned. It has to be noted, however, that the results obtained in this example remain in a numerically stable region.

Figure 3.7 shows the error on the evaluation set vs. the shape parameter by using $C^{4}$ Matérn kernel without (continuous lines) and with (discontinuous lines) enforced anisotropy, and for different sizes of the training set. Coherently with what observed for Sobol's $G$-function (Section 3.4.1), when anisotropy is enforced the error is less sensitive to the size of the training set and almost independent of the shape parameter, which can be left at its nominal value. Table 3.9 further presents some results of the approximation for the anvil's diameter increment. Here the accuracy improvements due to anisotropy are numerically quantified. Such effect, as in Figure 3.7, becomes more relevant when the training set is small.

Regardless of the value assigned to the shape parameter, when the radial kernel is enhanced with optimised anisotropic metrics, the error is drastically reduced. Additionally, as Figure 3.8 shows, the value of the shape parameter is almost irrelevant.

One can consider to optimise first the anisotropic metrics and then, with these fixed, perform an additional optimisation on the shape parameter, trying to further reduce the error. The results, done for example on a training set of 64 samples with optimised anisotropic metrics and an initial shape factor $\epsilon=0.5$, 


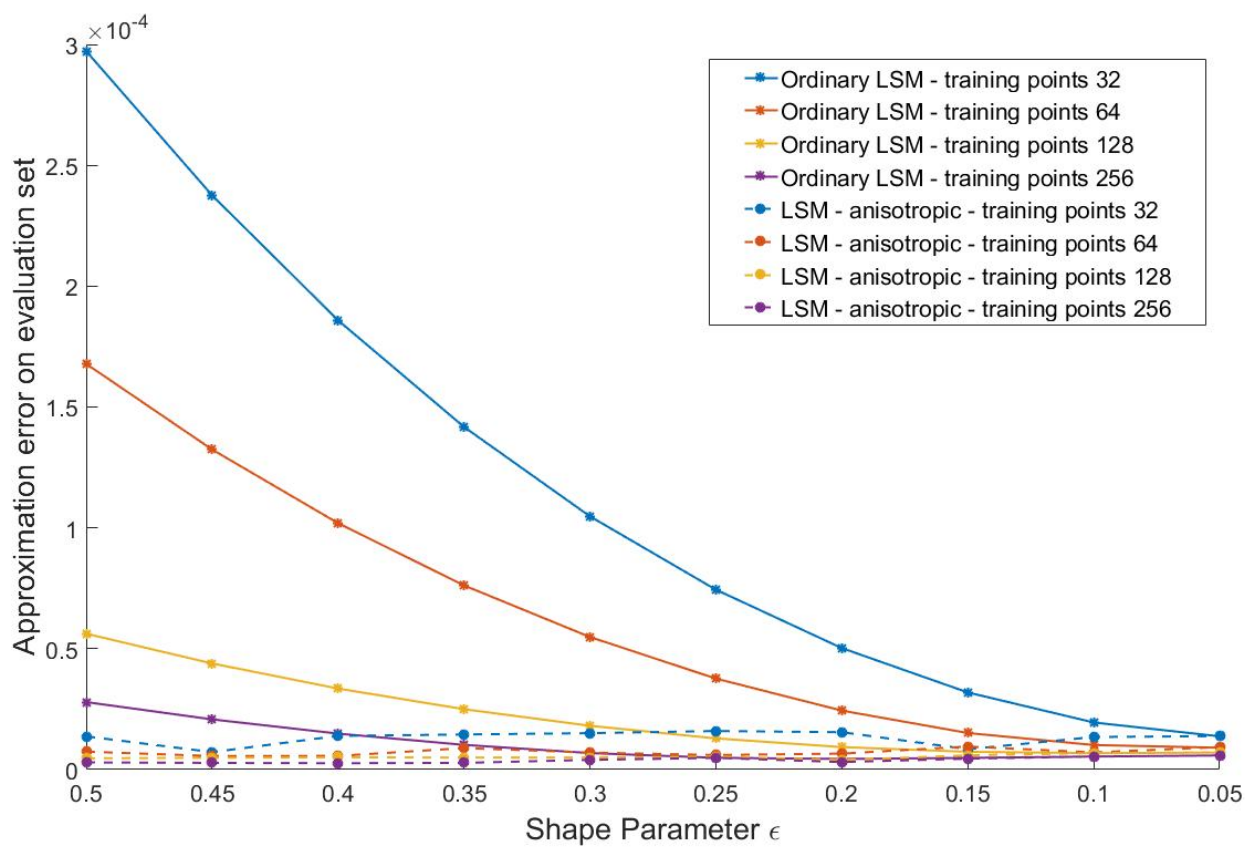

Figure 3.7: Taylor's test: anvil's diameter increment. Errors obtained by using radial kernels (continuous lines) and by enforcing anisotropic metric (discontinuous lines) vs. decreasing value of the shape parameter $\epsilon$. Four sizes are considered for the training set: 32, 64, 128, 256 points. In the case of enforced anisotropy, the error is less sensitive to the size of the training set and almost independent from the shape parameter.

\begin{tabular}{lcccc} 
Training Set $N_{s}$ & 32 & 64 & 128 & 256 \\
\hline$\epsilon=0.5 ; \gamma=1$ & $2.97 \times 10^{-4}$ & $1.68 \times 10^{-4}$ & $5.62 \times 10^{-5}$ & $2.79 \times 10^{-5}$ \\
$\epsilon=0.5 ; \gamma=$ optima & $1.37 \times 10^{-5}$ & $7.42 \times 10^{-6}$ & $4.65 \times 10^{-6}$ & $2.97 \times 10^{-6}$ \\
$\Delta E r r_{1}$ & $95 \%$ & $95 \%$ & $92 \%$ & $89 \%$ \\
\hline$\epsilon=0.3 ; \gamma=1$ & $1.05 \times 10^{-4}$ & $5.48 \times 10^{-5}$ & $1.81 \times 10^{-5}$ & $6.82 \times 10^{-6}$ \\
$\epsilon=0.3 ; \gamma=$ optima & $1.51 \times 10^{-5}$ & $7.03 \times 10^{-6}$ & $4.96 \times 10^{-6}$ & $4.01 \times 10^{-6}$ \\
$\Delta E r r_{2}$ & $86 \%$ & $87 \%$ & $72 \%$ & $41 \%$ \\
\hline$\epsilon=0.1 ; \gamma=1$ & $1.95 \times 10^{-5}$ & $1.02 \times 10^{-5}$ & $6.80 \times 10^{-6}$ & $5.40 \times 10^{-6}$ \\
$\epsilon=0.1 ; \gamma=$ optima & $1.35 \times 10^{-5}$ & $7.09 \times 10^{-6}$ & $5.10 \times 10^{-6}$ & $4.39 \times 10^{-6}$ \\
$\Delta E r r_{3}$ & $31 \%$ & $30 \%$ & $25 \%$ & $19 \%$ \\
\hline
\end{tabular}

Table 3.9: Anvil's diameter increment. For training sets of four different sizes, blocks report the errors obtained by considering only the shape parameter $\epsilon$ with or without optimised anisotropic metrics. In the third line of each block of results, the relative improvement on the error is shown. 
gives an error equal to $7.36 \times 10^{-6}$ and an optimal $\epsilon=0.4843$. Comparing this result with the error shown in Table $3.9,7.42 \times 10^{-6}$, one concludes that the optimisation of the shape parameter has no significant effect on the error, as expected from the previous observations.

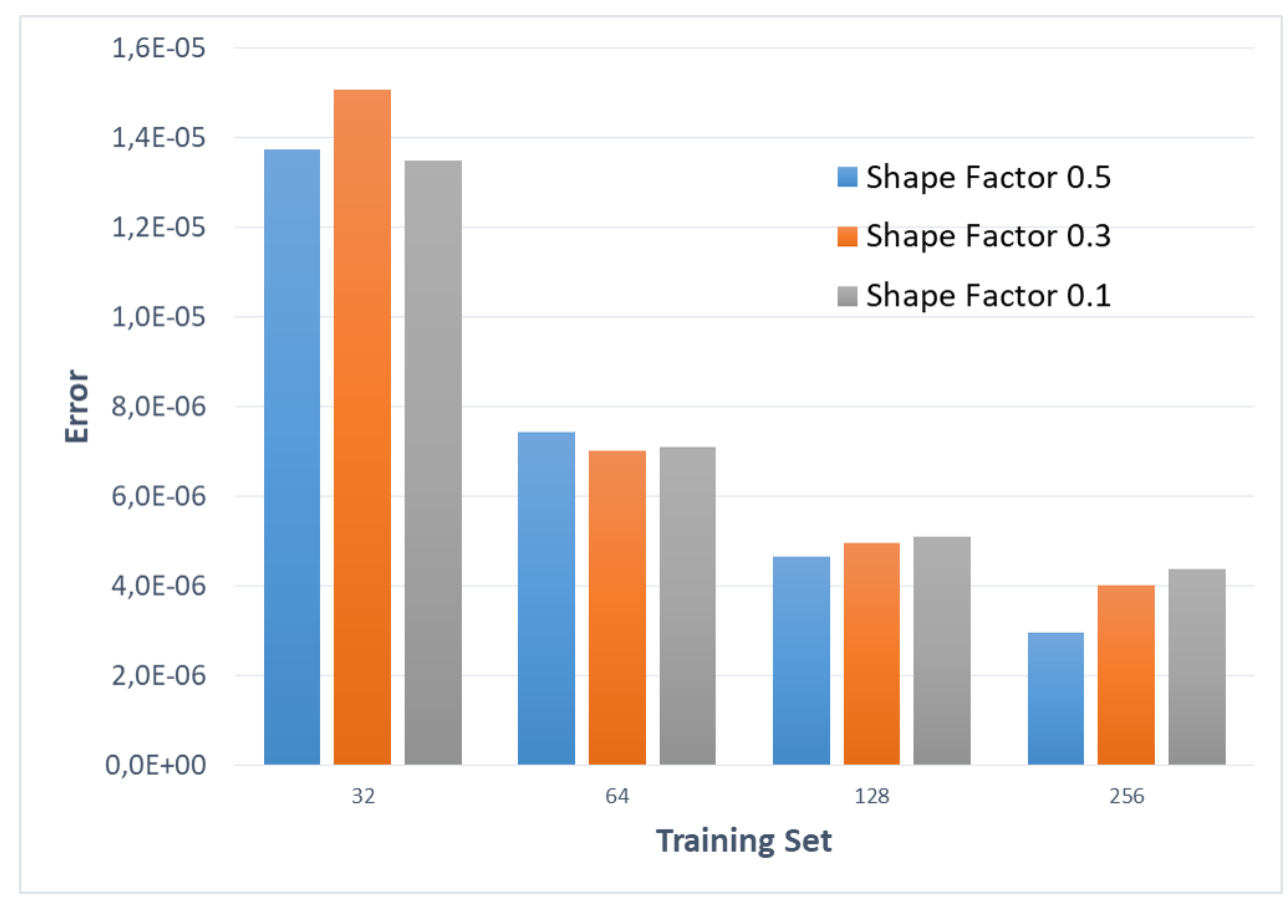

Figure 3.8: Errors obtained by using radial kernels with optimised anisotropic metrics vs. wider training set. For each set three values of the shape parameter are considered, i.e, 0.5 (blue), 0.3 (orange) and 0.1 (grey) bars. The value assigned to the shape parameter does not have a relevant effect on the error when the metrics are optimised.

When the same procedure is applied to generate a meta-model for estimating the probability density function of the anvil's length reduction, similar conclusions are reached.

Finally a meta-model with optimized anisotropic metrics and based on 64 training points is selected to estimate the global sensitivities of both QoIs.

\section{Global sensitivity results.}

The sensitivity results are summarized in the pie charts of Figure 3.9, for the diameter increment, and Figure 3.10, for the length reduction. The first order sensitivities cover approximately $100 \%$ of the output variance, which means that interactions among the input parameters do not play an important role on the outputs variability. Two influential parameters exist: the velocity of the impact 
and the material parameter $B$, which affect, respectively, $85 \%$ and $13 \%$ of the diameter increment variance and $86 \%$ and $9 \%$ of the length reduction variance. This is a remarkable result because it shows that the remaining parameters, according to the prescribed distributions and to the selected QoIs, are almost noninfluential, and can be kept at their nominal values. The study of the sensitivity indices can be seen as a dimensional reduction tool. In fact, one can think to build up even more efficient meta-models including only two relevant stochastic inputs. In principle, due to the low dimension of the scatter function, also the training set needed to generate the meta-model is expected to be smaller.

To conclude this study, Figures 3.11 and 3.12 show respectively the probability density functions of the diameter increment and the length reduction. Both distributions can be assimilated to Gaussians. Details about the mean and the variance are reported in the legend of these figures. In particular, the length's reduction fits quite well the experimental results presented by Johnson [75] and summarized in the following table:

\begin{tabular}{ccc} 
Velocity $[\mathrm{m} / \mathrm{s}]$ & $L_{i}[\mathrm{~mm}]$ & $L_{f}[\mathrm{~mm}]$ \\
\hline 130 & 25.4 & 20.59 \\
148 & 25.4 & 19.71 \\
190 & 25.4 & 17.22 \\
\hline
\end{tabular}

Table 3.10: Experimental results presented in [75] for the impact tests of a copper cylinder $\left(L_{i}:\right.$ initial length of the anvil; $L_{f}:$ final length). When the impact velocity is $190 \mathrm{~m} / \mathrm{s}$, the experimentally measured length reduction is very close to the mean value of the Gaussian of Figure 3.12 (i.e. $\mu=8.114 \mathrm{~mm}$ ). 
First Order Sensitivity Pie

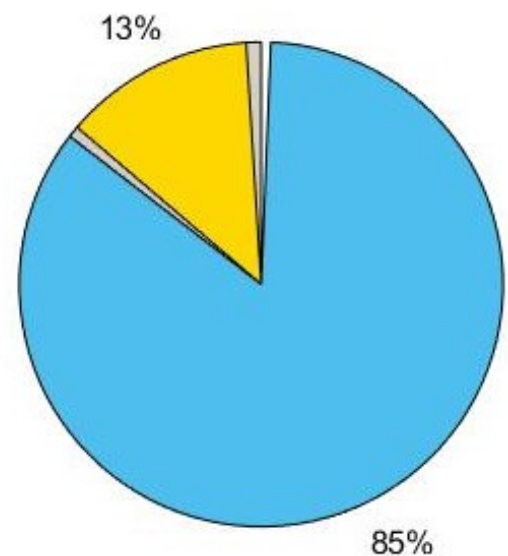

Total Order Sensitivity Pie
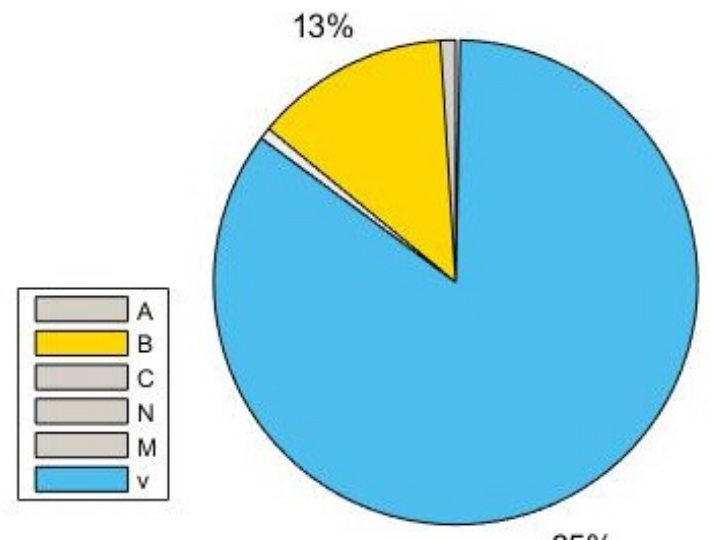

$85 \%$

Figure 3.9: Diameter increment - sensitivity indices results: according to the prescribed distribution only two outputs, the velocity and the material parameter $B$, have influence on the output variance of the diameter increment. All the rest can be fixed at its nominal value.
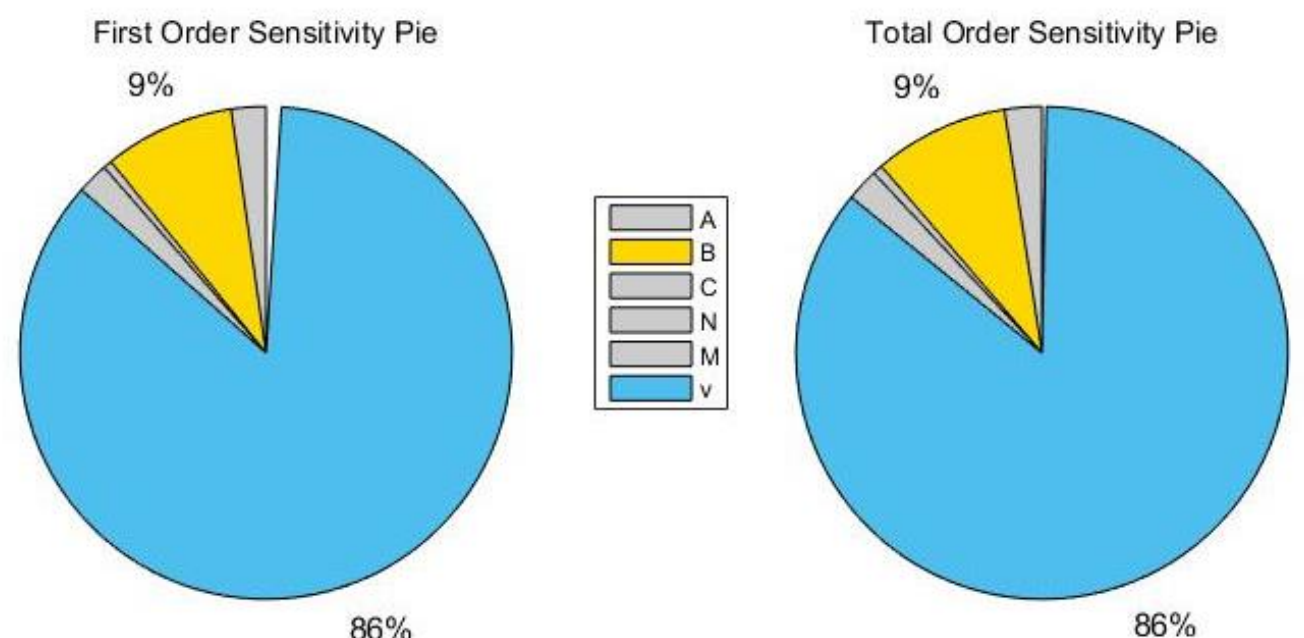

Figure 3.10: Anvil's length reduction - sensitivity indices results: for this QoI the velocity and the material parameter $B$ influence the output's variance the most. 


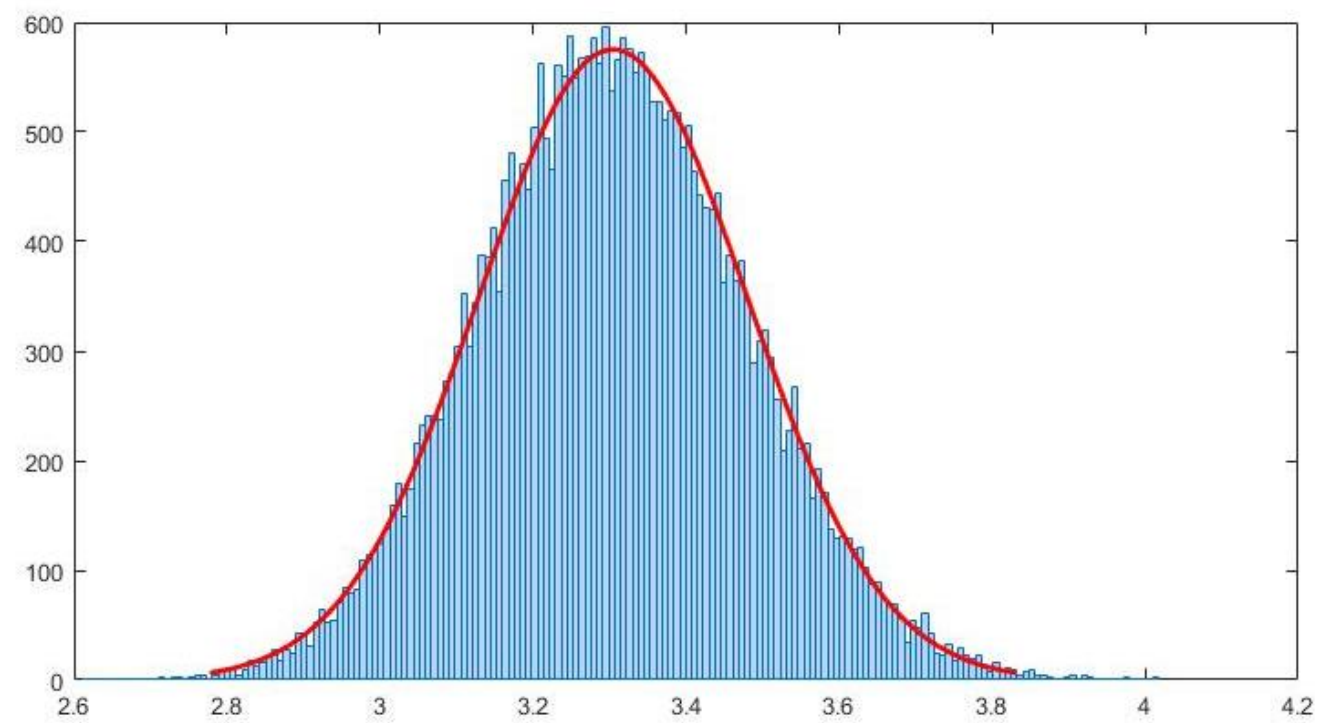

Figure 3.11: Probability density function of the diameter increment after the impact. The distribution is Gaussian with a mean $\mu=3.304$ and a standard deviation $\sigma=0.175$.

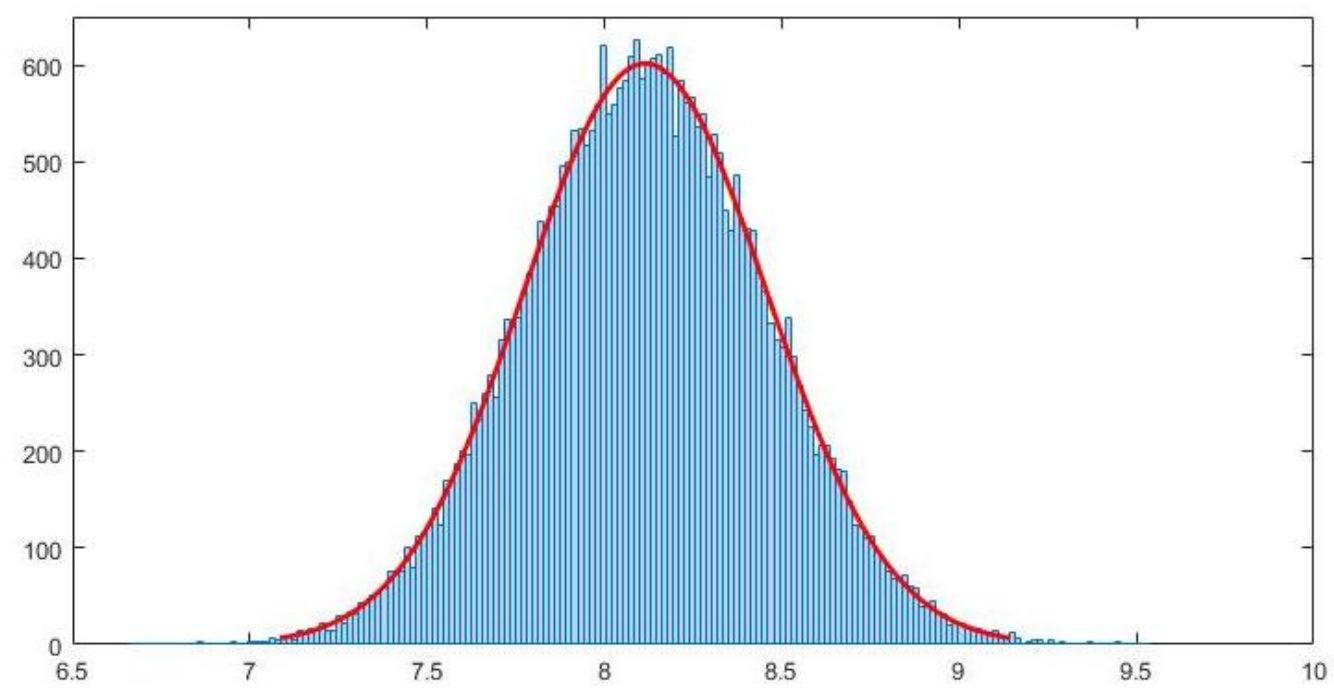

Figure 3.12: Probability density function of the length reduction after the impact. The distribution is Gaussian with a mean $\mu=8.114$ and a standard deviation $\sigma=0.343$.

\section{Interaction between velocity and material's parameters.}

In this section the relationship between the material's parameters is investigated in order to understand the interaction among them and with respect to 
velocity. A further extension of this section can be found in [78].

Figures 3.9 and 3.10 show that velocity is so relevant that it masks the effects of other parameters, but B. Additionally, by comparing the first and total indices, one can see that impact velocity has very low interactions with the material's parameters, i.e. its effect is additive. If velocity is excluded from the set of random parameters, the relative relevance of the remaining variables, which are now only the five material parameters, should be kept similar at different (but fixed) velocities.

To demonstrate that a cloud of 100 simulations is run for each value $v=$ [200 230260290320$][m / s]$. Figure 3.13 shows the results. Each multi-lineal curve corresponds to a given a set of parameters over different velocity. If one moves from a set of material parameters to another one, the slope of the multilineal branch, slightly changes because a little interaction exists with the velocity. The results suggest that, given a set of parameters, one can obtain the length reduction (and equivalently the diameter increment) at each velocity by a simply linear interpolation.

As additional prove, a meta-model is constructed at each velocity and is used to perform the UQ process and GSA. It is shown in table 3.11, where mean, variance, first and total effects are provided. There is not significant change in Sobol's sensitivities vs. velocities. As well, first order effects almost cover the $100 \%$ of the total variance, the more relevant being A, B and C. N and M can be fixed at their nominal values.

Now, a further evaluation set of 500 simulations which include the velocity as random uniform variable $V \sim \mathcal{U}[200,320]$ is obtained. Figure 3.14 shows the results of comparing such simulations with those obtained by using the metamodels. The five surrogates consider first all the parameters of the evaluation set, i.e. 500 simulations, but velocity, and hence get the results at the five fixed velocity at which they have been constructed. Then, according to the interpolation procedure shown in figure 3.13, the results are obtained at the desired velocities. The good agreement of the results is shown in figure 3.14, where each point of the scatter plot is a simulation point (blue) or an interpolated meta-models result (orange).

Figure 3.15 shows the distribution of the error, defined according to the expression

$$
\operatorname{Err}=\frac{\left\|\Delta L_{\text {sim }}-\Delta L_{\text {int.MM }}\right\|}{N_{\text {sim }}\left\|\Delta L_{\text {int.MM }}\right\|} .
$$

This section provides, to the best of my knowledge, the first UQ and GSA analysis of Taylor's impact test. The results obtained can help the parameter 


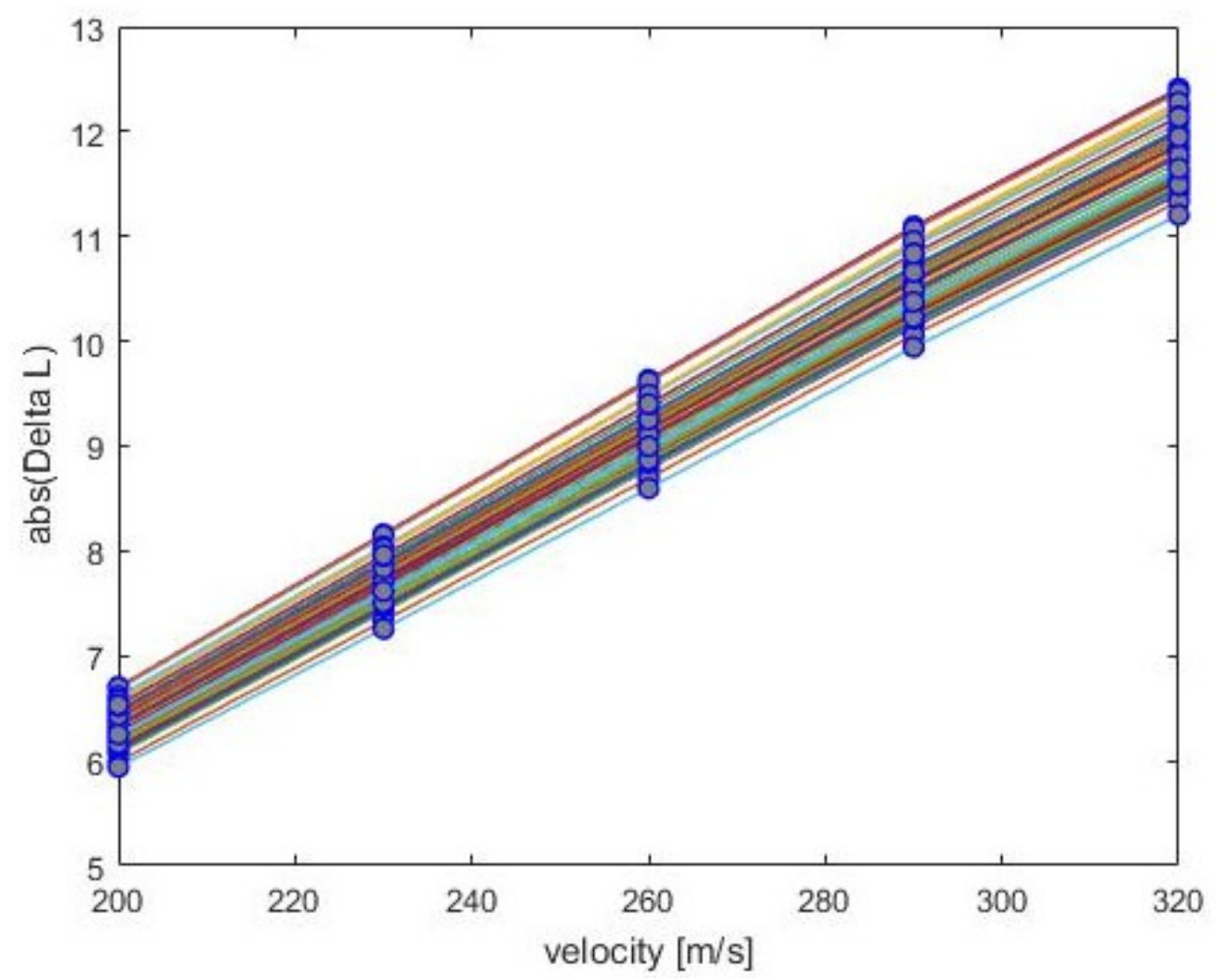

Figure 3.13: The trend of the length reduction vs. velocity is multi-lineal. A little interaction of velocity with the other parameters arises because the slope of the curves is not exactly the same when the set of input parameters is changed. Given a set of parameters by using the multi-lineal curves, one can obtain the length reduction (and equivalently the diameter increment) at each velocity by a simply linear interpolation.

fitting for constitutive models beyond Jonhson-Cook's, and guide the formulation of effective meta-models for other similar complex experimental setups.

\subsection{Conclusions and future work}

The present chapter has focused on the design of meta-models based on radial kernels for the simulation of complex mechanical systems. More specifically, the properties of radial kernels with anisotropic metric are discussed in detail. These surrogates are introduced as an opportunity for obtaining improved performance, in terms of efficiency and predictiveness, particularly when a limited set of model evaluations is available. The link between anisotropic metrics and Sobol's sensitivities is explored and quantified using GSA. A detailed methodology is pre- 


\begin{tabular}{cccccc}
\hline Velocity $[\mathrm{m} / \mathrm{s}]$ & 200 & 230 & 260 & 290 & 320 \\
\hline Mean $(\Delta L)[\mathrm{mm}]$ & 6.446 & 7.854 & 9.277 & 10.693 & 11.987 \\
$\mathrm{~V}(\Delta L)[\mathrm{mm}]$ & 0.233 & 0.273 & 0.313 & 0.349 & 0.366 \\
\hline Sobol Indices & & & & & \\
$S_{1}(\mathrm{~A})$ & $28.86 \%$ & $27.55 \%$ & $26.26 \%$ & $25.04 \%$ & $24.10 \%$ \\
$S_{2}(\mathrm{~B})$ & $41.64 \%$ & $43.69 \%$ & $45.68 \%$ & $47.56 \%$ & $48.43 \%$ \\
$S_{3}(\mathrm{C})$ & $22.29 \%$ & $22.86 \%$ & $23.34 \%$ & $23.73 \%$ & $24.45 \%$ \\
$S_{4}(\mathrm{~N})$ & $7.05 \%$ & $5.76 \%$ & $4.57 \%$ & $3.52 \%$ & $2.85 \%$ \\
$S_{5}(\mathrm{M})$ & $0.04 \%$ & $0.04 \%$ & $0.04 \%$ & $0.04 \%$ & $0.04 \%$ \\
\hline$S T_{1}(\mathrm{~A})$ & $28.94 \%$ & $27.62 \%$ & $26.34 \%$ & $25.12 \%$ & $24.21 \%$ \\
$S T_{2}(\mathrm{~B})$ & $41.62 \%$ & $43.66 \%$ & $45.66 \%$ & $47.56 \%$ & $48.44 \%$ \\
$S T_{3}(\mathrm{C})$ & $22.33 \%$ & $22.90 \%$ & $23.38 \%$ & $23.79 \%$ & $24.52 \%$ \\
$S T_{4}(\mathrm{~N})$ & $7.11 \%$ & $5.82 \%$ & $4.63 \%$ & $3.57 \%$ & $2.90 \%$ \\
$S T_{5}(\mathrm{M})$ & $0.04 \%$ & $0.04 \%$ & $0.04 \%$ & $0.04 \%$ & $0.04 \%$ \\
\hline
\end{tabular}

Table 3.11: JC model - length decrement: 5 surrogates are generated to evaluate the Sobol's Indices at 5 different impact velocity. Mean, variance and sensitivities, main and total effects, are provided in the table. Results confirm that the relative relevance of the material's parameters is slightly sensitive to the change in velocity. Additionally, the main effect cover already almost the $100 \%$ of the total variance, hence no relevant interactions among material's parameters exist. Finally, the parameters $\mathrm{N}$ and $\mathrm{M}$ can be fixed at their nominal values.

sented that can be used to systematically construct quasi-optimal meta-models. To showcase the impact of these improved kernels, a 15 dimensional $G$-Sobol's function and two mechanical applications are studied: a textbook example involving the motion of a piston and Taylor's elastoplastic impact test. In all three cases I provided guidelines for the design of surrogates and their use together with Sobol's LDD and GSA. Using the ideas set forward in this thesis, significant improvements in terms of computational cost and predictiveness of the metamodels, are obtained.

I find particularly interesting for the mechanical engineering community the sensitivities calculated with these meta-models for the material model of Johnson and Cook. The identification of the most relevant material parameters can help to improve the experimental set-up for high-velocity impact tests, and help in steering experimental campaigns for parameter fitting.

Further improvement avenues could consider the optimal placement of the centers of the kernel functions and the investigation of compactly supported radial kernels. The first option will result in a reduced number of columns in the kernel 


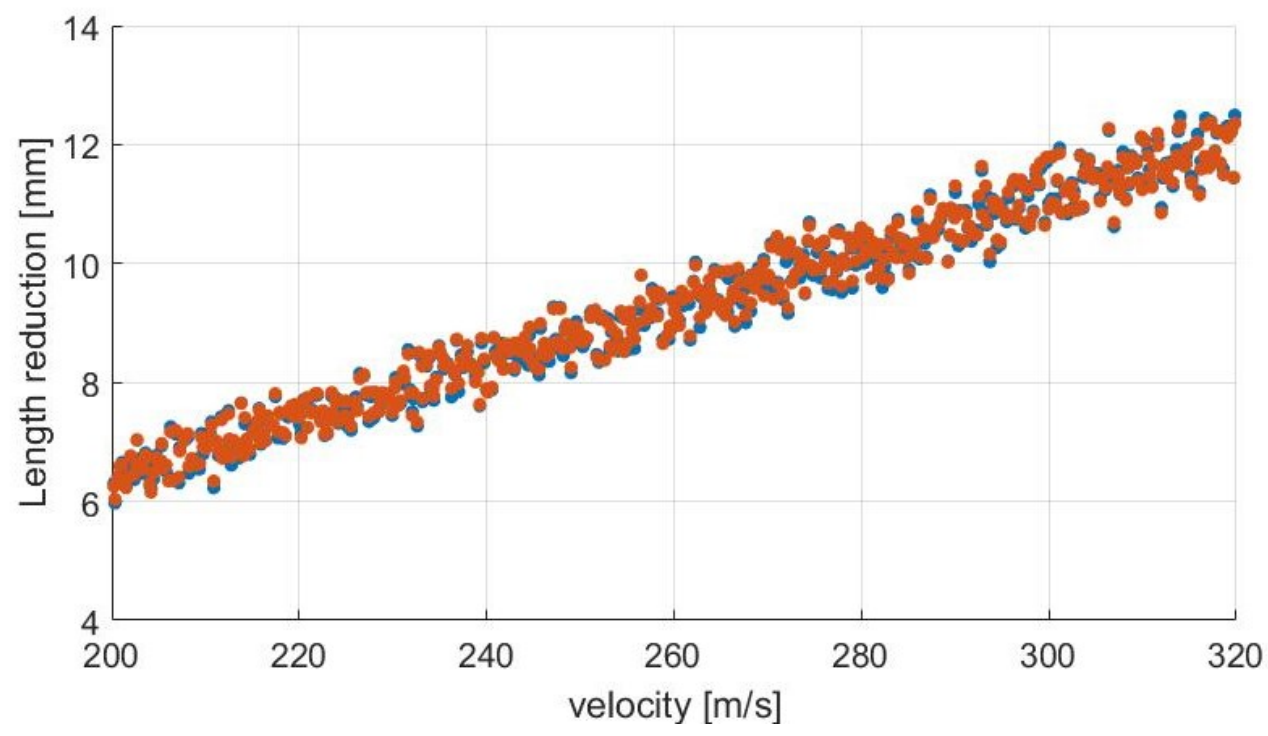

Figure 3.14: Figure shows the comparison between the results obtained by simulations and those obtained by employing the meta-model interpolation approach. Simulation points are plotted in blue, while interpolated meta-models results are in orange.

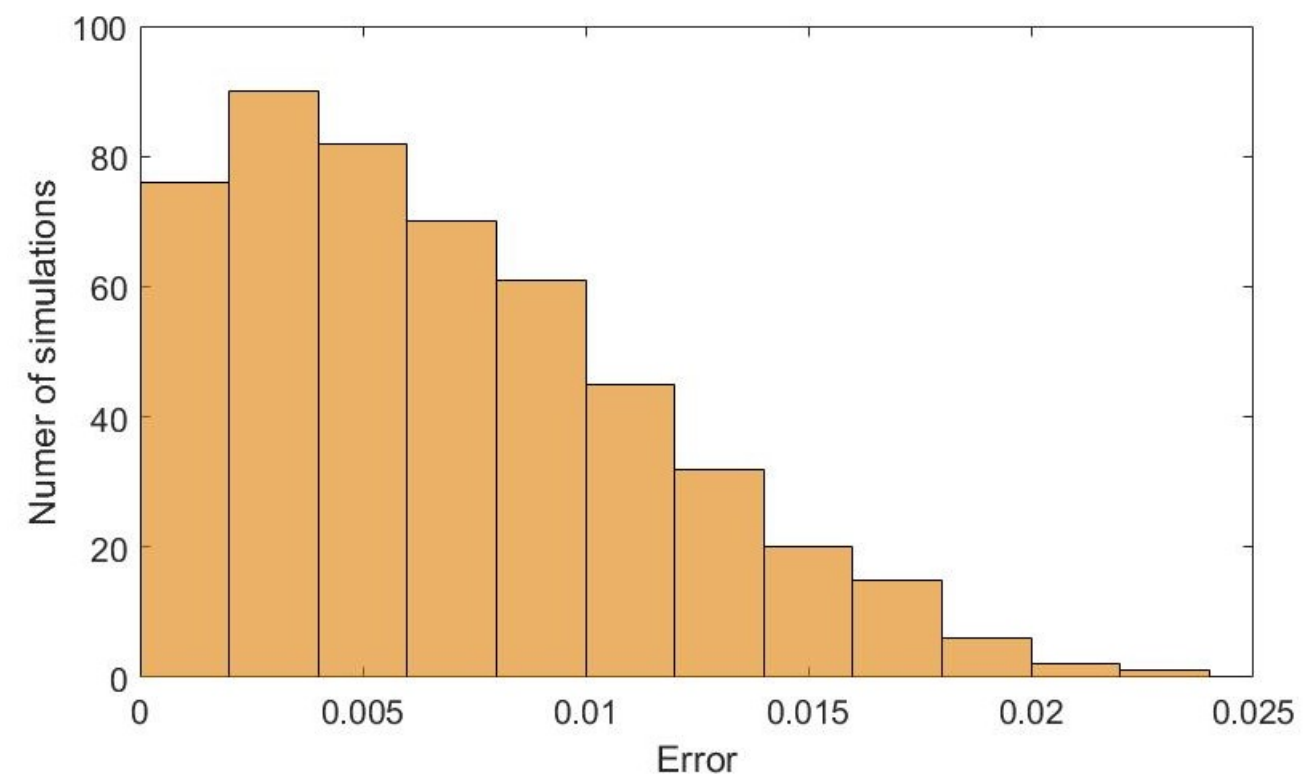

Figure 3.15: Histogram of the error over the 500 simulations. The mean error is 0.006 .

matrix and the latter will improve its sparsity. These extensions are left for future works. 


\section{Chapter 4}

\section{Reduction Order Methods and Meta-models}

\subsection{Introduction}

Assembled structures in the aerospace industry are often connected through discontinuous and nonlinear junctions whose mechanical properties are seldom well-characterized experimentally. From a modeling point of view, they are often reduced to a set of linear springs with some stiffness and damping values. It happens that, during the project, these values are reviewed, discussed, and fine-tuned to get a reasonable or expected dynamic response [79, 80]. Local parameters are changed one at a time to analyze their effect on the outputs: the higher the number of input parameters, the more tedious the task is and the more complex the understanding of the results as well. In particular, interfaces play a key role in defining the dynamics of components and systems but considering properly their behavior can be a very complex task. This is especially true for dynamic studies [81, 82]. Their behavior, often nonlinear, is intrinsically uncertain and as consequence, making the dynamic response of the connected structures stochastic.

This chapter looks at the UQ process as a systematic approach to establish, quantitatively and qualitatively, how the uncertain behavior of interfaces among components affects the overall dynamics of a structure. In the UQ process also the GSA are included, which allows to rank the input parameters according to their influence on the variance of the stochastic QoIs. These QoIs are precisely those outputs which allow to understand and make decisions about the structure.

Some researchers have already investigated the uncertainty within the joints 
of assembled structures [83, 84], as well as in the assembly and manufacture of certain structural applications such as micro-electromechanical systems [85]. Most pragmatic and industrial approaches to UQ are based on sampling the deterministic model according some specific computer design, i.e., the Monte Carlo method, in order to produce a cloud of output points, which can then be used to obtain sample-based statistics. In any case, but especially for computationally expensive simulations, the direct use of Finite Element (FE) models can be extremely time-consuming. Hence, usually, a smaller set of simulations is used to generate first a meta-model, which is used to complete the UQ analyis [86, 87]. However, when the dynamic response of a structure is affected by strong nonlinearities, for example when the system has free-plays or includes rubber-like materials, the number of simulations required to generate a reliable meta-model can be still considerably high. The main reason is that these types of nonlinearities affect the smoothness of the high-dimensional response surface, which often cases will present discontinuities.

\subsubsection{Outline of the chapter}

In light of the previous observations, and considering that often junctions are represented by lumped elements, the Structural Dynamic Modifications Method (SDMM) $[88,89]$ seems a sensible approach for reducing the computational cost of the simulations. The principle behind modification methods is to run the FE Model just once, extracting the modal information at the required degrees of freedom (dofs) and then to update the equations, expressed in modal coordinates, through a set of modifications. While a large body of literature exists in the field of linear modifications, works dealing with nonlinear modifications are significant fewer, i.e. [90, 91]. The formulation of the NL SDMM followed in this thesis has been introduced by Menga and Hernández $[52,53,54]$ and is presented in Section 4.2. It has been proven to be a reliable and efficient approach to reduce drastically the computation burden associated with one deterministic nonlinear simulation when the global dynamic behavior depends on localized sources of nonlinearity, which can be modeled via lumped elements, i.e., elements connecting pairs of dofs.

In the current chapter, the NL SDMM is used to produce efficiently the initial set of simulations which will be used for generating the meta-model. Then the surrogate, based on a multivariate PCE, can be used to generate a broader sample of output points and obtaining the sensitivity indices.

A brief review of the PCE meta-modeling technique and of variance-based 
GSA is given in Section 4.3. Also, the mathematical link between the coefficients of the polynomial expansion and the sensitivity indices is discussed, although in a non-exhaustive fashion. The interest reader can refer for example to the work of Saltelli and Ratto [21] in the context of GSA and to the publications of Sudret [4] in the field of PCE.

Section 4.4 describes an industrial application of the previous ideas, combined in a systematic approach. The example considers an aircraft component whose dynamic behavior is affected by free-play interfaces, which are intrinsically uncertain.

Finally, Section 4.5 summarizes the main conclusions and proposes some possible future work.

\subsection{Nonlinear Structural Dynamic Modifications}

In this section the theory behind the NL SDMM is reviewed. The modal matrix and the eigenvalues are assumed to be available from the FE modal analysis of the model. Also, I consider that the only source of nonlinearity in the model is due to the interfaces between components. In this section the equation in the case of a nonlinear stiffness update is formulated, but the same approach can be followed for mass and damping modifications.

The initial stiffness of the spring elements used in the FE model for obtaining the modal properties is called underlying linear stiffness, whose value, with reference to the Figure 4.1, is denoted $k_{1}$. Starting from the modal information of the model, one wants to update the dynamic equations to take into account the nonlinear stiffness modifications. Then, the modal base of the linear model is fixed, i.e., the FE model is run just once, and the nonlinear response is the result of an updating process, irrespective of the type of nonlinearities that are incorporated.

The dynamic equilibrium in time-domain can be expressed as:

$$
M \ddot{u}(t)+C \dot{u}(t)+K u(t)=F(t),
$$

where $M, C, K$ are, respectively, the mass, damping, and stiffness matrix, and $F$ is the vector of external forces. The eigenvalues and eigenvectors of the system are found by solving the problem:

$$
(K-\lambda M) \Phi=0
$$


where $\lambda$ is a diagonal matrix with entries equal to the eigenvalues of the system, and $\Phi$ is the modal matrix. The $i$-th column of the modal matrix $\Phi$ is the eigenvector corresponding to the eigenvalue $\lambda_{i}$.

The displacements of the system can be expressed as a linear combination of the eigenvectors weighted by some factors $q(t)$, which are called modal coordinates, that is:

$$
u(t)=\Phi q(t) .
$$

When the modal matrix is normalized with the mass, Eq. (4.1) can be expressed in the well-known modal form:

$$
I \ddot{q}(t)+\tilde{C} \dot{q}(t)+\Lambda q(t)=\tilde{F}(t)
$$

where $I, \tilde{C}$, and $\Lambda$ are now the normalized mass, damping and stiffness matrix, respectively, and $\tilde{F}$ is the vector of forces. In Eq. (4.4) the normalized mass matrix is the identity, and the normalized stiffness matrix is a diagonal matrix whose terms are the eigenvalues.

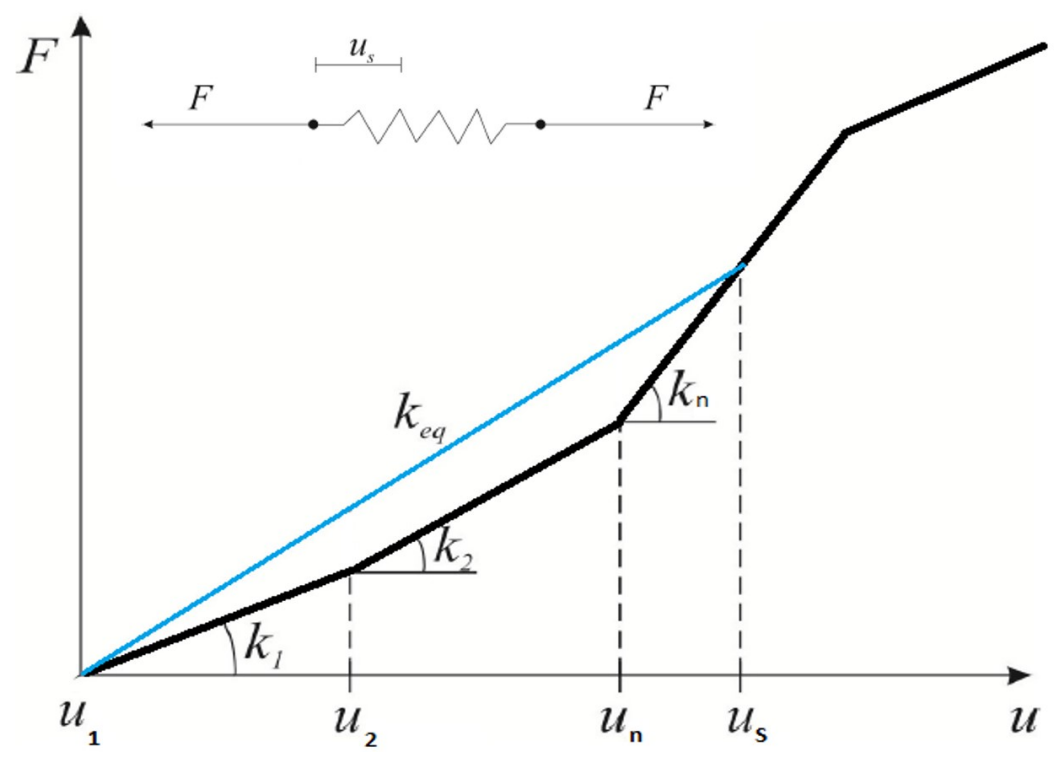

Figure 4.1: Multi-linear force-displacement curve.

With reference to Figure 4.1, when a relative displacement $u_{s}$ is applied between the two dofs of the spring, according to the force-displacement curve, the internal force is:

$$
F_{s}=k_{1}\left(u_{2}-u_{1}\right)+k_{2}\left(u_{3}-u_{2}\right)+\ldots+k_{n-1}\left(u_{n}-u_{n-1}\right)+k_{n}\left(u_{s}-u_{n}\right) .
$$


The same force can be obtained with a secant stiffness given by:

$$
k_{e q u}=\frac{\sum_{i=1}^{n-1} k_{i}\left(u_{i+1}-u_{i}\right)+k_{n}\left(u_{s}-u_{n}\right)}{u_{s}} .
$$

Considering an underlying linear stiffness value $k_{1}$, the additional contribution to the current stiffness is:

$$
\Delta k=k_{e q u}-k_{1},
$$

and the modification matrix is of the type:

$$
\Delta k_{\text {mod }}=\left[\begin{array}{cc}
\Delta k & -\Delta k \\
-\Delta k & \Delta k
\end{array}\right]
$$

When $N_{k}$ lumped elements are involved in the modification, the final matrix can be obtained as:

$$
\Delta K_{\text {mod }}=\sum_{m=1}^{N_{k}} \Delta k_{m o d}^{m}
$$

which can be normalized by the modal matrix of the underlying linear system:

$$
\Delta \tilde{K}_{\text {mod }}=\Phi^{T} \Delta K_{\text {mod }} \Phi
$$

Hence, the update stiffness matrix of the structure is:

$$
\Lambda_{\text {mod }}=\Lambda+\Delta \tilde{K}_{\text {mod }}
$$

and Eq. (4.4) can be re-written as:

$$
I \ddot{q}(t)+\tilde{C} \dot{q}(t)+\Lambda_{m o d} q(t)=\tilde{F}(t) .
$$

Eq. (4.12) needs to be solved by an iterative method which has to take into account that $k_{\text {equ }}$, and consequently $\Lambda_{\text {mod }}$, are nonlinear functions of the relative displacements of the springs, which, through the modal matrix $\Phi$, depend on the modal displacements. In this chapter the state space form of Eq. (4.12) has been implemented in MATLAB ${ }^{\circledR}$ and solved with the function ODE45, which uses an explicit Runge-Kutta integration procedure. Once the nonlinear modal displacements are obtained, the physical ones, for all the dofs considered in the modal matrix, can be evaluated by Eq. (4.3). I stress the fact that this approach is computationally very efficient: the linear FE Model is run just once in order to obtain the modal matrix, and usually the number of significant modes is much smaller than the dofs of the model. Additionally, the dofs involved in the up- 
dating process are usually a few dozen: the dofs where the loads are applied, those ones where the outputs are required, and finally those ones involved in the modifications.

\subsection{Uncertainty Quantification}

UQ can be thought as a process with three fundamental phases, namely, pre-processing, uncertainty propagation, and post-processing. The first phase aims to select which input parameters are the source of uncertainties, and to prescribe on them some appropriate probability distribution. The propagation of the uncertainties aims to characterize the distributions of the QoIs for a given distribution of inputs. That is obtained, in non-intrusive methods, by sampling the deterministic model according to some specific sampling technique. Then, the cloud of results can be post-processed in order to obtain sample-based statistics. The most common design is based on MC sampling, which relies on pure randomness. Due its randomness, some points can be clustered closely, while other regions within the space may not contain any samples. Hence, especially when working in high dimensions, most researchers seem to agree that generic, near-uniform, distribution of the data sites is a better strategy. Several sampling strategies try to cover this need, such as the LHD and the LDD. LDD, in particular, generates a deterministic sequence of quasi-random numbers which fill uniformly the unit hypercube. In this chapter, Sobol's LDD is considered [20]. It was the first digital sequence, operates in base-2, and has been found by several researchers, i.e. Pianosi [22], that this choice out-performs the classical MC and LHD methods being, in average, more efficient and having a faster convergence rate. For complex systems where, possibly, a very large set of inputs is considered uncertain, it is often necessary to generate first a meta-model and then, using it, complete the analysis.

\subsubsection{Meta-model: Polynomial Chaos Expansion}

The original Hermite PCE, also known as homogeneous chaos, was first derived by Wiener [92] for the spectral representation of any stochastic response in terms of Gaussian random variables. Later, Askey [93] studied the orthogonal properties of the Hermite polynomials with respect to the Gaussian probability density function, providing the well-known Wiener-Askey scheme. But only in 2003 Xiu [94] extended the method to others random distributions as summarised in Table 4.1. To ensure optimal convergence properties of the PCE, the polyno- 
mials are required to be orthogonal with respect to a weight function, which is representative of the probability distribution of the QoIs.

\begin{tabular}{lccc}
\hline Orthogonal polynomial & Weight function & PDF & Density function \\
\hline Hermite $H_{e}(x)$ & $e^{\frac{-x^{2}}{2}}$ & Normal & $\frac{1}{\sqrt{2 \pi}} e^{\frac{-x^{2}}{2}}$ \\
Legendre $P_{n}(x)$ & 1 & Uniform & $\frac{1}{2}$ \\
Laguerre $L_{n}(x)$ & $e^{-x}$ & Exponential & $e^{-x}$ \\
Jacobi $P_{n}^{\alpha, \beta}(x)$ & $x^{\alpha} e^{-x}$ & Beta & $\frac{x^{\alpha} e^{-x}}{\Gamma(\alpha+1)}$ \\
\hline
\end{tabular}

Table 4.1: Correspondence between orthogonal polynomials and PDF.

To introduce the notation that will be employed later, let us briefly describe next the standard least square approximation problem: if a model is sampled at $N_{s}$ points of the high-dimensional space $\mathcal{S} \equiv \mathbb{R}^{N_{p}}$, the generic output $Y_{n}=Y\left(X_{n}\right)$ evaluated at the input point $X_{n}=\left[X_{n 1}, X_{n 2}, \ldots, X_{n N p}\right]$, with $n \in\left[1,2, \ldots, N_{s}\right]$ and $N_{p}$ the total number of input parameters considered, can be approximated by a linear combination of $N_{k}$ basis or kernel functions $\Psi_{k}: \mathcal{S} \rightarrow \mathbb{R}$ as

$$
Y\left(X_{n}\right) \simeq \sum_{k=1}^{N_{k}} \beta_{k} \Psi_{k}\left(u_{n}\right) .
$$

In this case, the basis functions are orthogonal polynomials with respect to a defined weight, and usually in as specific domain. For example, Legendre polynomials are orthogonal with respect a uniform probability density function in the domain $[-11]$. Hence, each $u_{n}$ is a normalised input random variable, and $X_{n}$ has to be mapped to the domain where the orthogonality is ensured.

This relation can be expressed more compactly using matrix notation as in

$$
Y(X) \simeq \Psi(u) \beta
$$

where $\Psi$ is the interpolation or kernel matrix, $Y$ is the matrix of the $N_{\text {out }}$ QoIs evaluated at $N_{s}$ sampled points and $X$ is the sample matrix.

Usually, $\Psi_{k}$ are multivariate polynomials that involve products of the onedimensional polynomials $\psi_{i}\left(u_{j}\right)$, where $i$ is the order of the polynomial and $u_{j}$ is the $j$-th component of the vector $u_{n}$. The multivariate expansion is obtained as a tensor product of the one-dimensional basis and consequently presents higherorder terms. These higher order terms are usually truncated considering for the multivariate basis the same maximum order $o$ of the one-dimensional polynomials they come from. 
Under these conditions, the total number of coefficients $N_{k}$ of the polynomial expansion in Eq. (4.13) is given by:

$$
N_{k}=\frac{\left(N_{p}+o\right) !}{N_{p} ! o !} .
$$

As an example, the first six one-dimensional Hermite polynomials are considered:

$$
\{\psi(u)\}=\left\{1, u, u^{2}-1, u^{3}-3 u, u^{4}-6 u^{2}+3, u^{5}-10 u^{3}+15 u, \ldots\right\},
$$

and the multivariate polynomials for a second-order expansion over two random variables, presented in Table 4.2. The higher order terms, such as $\Psi_{6}(u)=$ $\psi_{2}\left(u_{1}\right) \cdot \psi_{1}\left(u_{2}\right)=u_{1}^{2} u_{2}$, are neglected.

\begin{tabular}{llll}
$\Psi_{0}(u)$ & $\psi_{0}\left(u_{1}\right) \cdot \psi_{0}\left(u_{2}\right)$ & $1 \cdot 1$ & 1 \\
$\Psi_{1}(u)$ & $\psi_{1}\left(u_{1}\right) \cdot \psi_{0}\left(u_{2}\right)$ & $u_{1} \cdot 1$ & $u_{1}$ \\
$\Psi_{2}(u)$ & $\psi_{0}\left(u_{1}\right) \cdot \psi_{1}\left(u_{2}\right)$ & $1 \cdot u_{2}$ & $u_{2}$ \\
$\Psi_{3}(u)$ & $\psi_{2}\left(u_{1}\right) \cdot \psi_{0}\left(u_{2}\right)$ & $\left(u_{1}^{2}-1\right) \cdot 1$ & $u_{1}^{2}-1$ \\
$\Psi_{4}(u)$ & $\psi_{0}\left(u_{1}\right) \cdot \psi_{2}\left(u_{2}\right)$ & $1 \cdot\left(u_{2}^{2}-1\right)$ & $u_{2}^{2}-1$ \\
$\Psi_{5}(u)$ & $\psi_{1}\left(u_{1}\right) \cdot \psi_{1}\left(u_{2}\right)$ & $u_{1} \cdot u_{2}$ & $u_{1} u_{2}$ \\
\hline
\end{tabular}

Table 4.2: Bivariate second order Hermite polynomials.

From Eq. (4.15) it can be noted that increasing the number of random variables or the order of the polynomial will cause a substantial increase in the number of terms $N_{k}$ of the PCE and the sample size $N_{s}$ required to find the coefficients of the expansion. Consequently, in the case of complex simulations, the computational cost could be unaffordable.

In this chapter the least square method is employed to estimate the unknown coefficients $\beta_{k}$ of the PCE. Considering Eq. (4.13) and minimizing the sum of the squares of the residuals:

$$
\beta=\arg \min _{\beta^{*} \in \mathbb{R}^{N_{k}}}\left\|\Psi \beta^{*}-Y\right\|^{2},
$$

the least square fitting gives a closed-form solution:

$$
\beta=\left(\Psi^{T} \Psi\right)^{-1} \Psi^{T} Y
$$

where $\Psi$ is assumed to have full column rank.

The number of input points $N_{s}$ must be higher than the number of unknown 
coefficients $N_{k}$ and, depending on the complexity of the response, the recommended number of samples may fluctuate. Following Hosder [95], $N_{s} \simeq 2 \cdot N_{k}$ has been employed.

\subsubsection{Global Sensitivity Analysis}

GSA defines a qualitative and quantitative mapping between the input variables and the QoIs. Sensitivity analyses are strictly related to the Uncertainty Propagation (UP) and they can be seen as complementary to each other: UP quantifying the uncertainty in the output of the model, while GSA focuses on apportioning the output's uncertainty to the different sources of uncertain parameters [21].

GSA allows to identify the most relevant parameters of the model, and score them according to their relevance in the QoIs. In this context, a sensitivity index is a measure of the influence of an uncertain quantity on an output variable. The most popular GSA techniques are based on the decomposition of the variance's output probability distribution and allow the calculation of Sobol's sensitivity indices. They refer to Sobol', because he formulated the indices from the decomposition of the variance proposed by the HDMR of Hoeffding' [39]. From a practical point of view, particular attention is paid to the first order and total order sensitivity indices, denoted respectively as $S_{i}^{I}$ and $S_{i}^{T}$, also called the main effects and the total effects. If a model $Y$ is a function of $N_{p}$ stochastic variables $X_{i}$ with $i=1, \ldots, N_{p}$, the main effects represent the contribution of each parameter $X_{i}$ to the output variance, or equivalently, the expected percentage reduction of the output variance $V(Y)$ obtained when the uncertainty of input parameter $X_{i}$ is eliminated. The total effects represent the total contribution of each input parameter considering its individual effect and its interaction with the remaining factors.

\subsubsection{Polynomial Chaos and Sobol's Indices}

According to the PCE theory, the mean and the variance of the QoIs can be estimated directly by means of the PCE coefficients. In fact, assuming that the polynomials are normalized with the variance, the following relationships hold:

$$
E(Y(X)) \approx \beta_{0}
$$




$$
V(Y(X)) \approx \sum_{k=1}^{N_{k}} \beta_{k}^{2} .
$$

In the context of multivariate polynomials, the concept of a multi-index $\alpha_{k}$ is often presented. This notation allows to describe the order of the multivariate terms from the tensor product of the univariate polynomials. Table 4.3 provides the multi-indices in the case of a second-order, bivariate PCE.

\begin{tabular}{|c|c|c|c|c|c|}
\hline \multirow{2}{*}{$\frac{\text { Multivariate PCE }}{\Psi_{0}(u)}$} & \multicolumn{3}{|c|}{ Multi-Index } & \multirow{2}{*}{$\frac{\text { Coefficients }}{\beta_{0}}$} & \multirow{2}{*}{$\begin{array}{l}\text { function } \\
1\end{array}$} \\
\hline & $\alpha_{0}$ & 0 & 0 & & \\
\hline$\Psi_{1}(u)$ & $\alpha_{1}$ & 1 & 0 & $\beta_{1}$ & $u_{1}$ \\
\hline$\Psi_{2}(u)$ & $\alpha_{2}$ & 0 & 1 & $\beta_{2}$ & $u_{2}$ \\
\hline$\Psi_{3}(u)$ & $\alpha_{3}$ & 2 & 0 & $\beta_{3}$ & $u_{1}^{2}$ \\
\hline$\Psi_{4}(u)$ & $\alpha_{4}$ & 0 & 2 & $\beta_{4}$ & $u_{2}^{2}$ \\
\hline$\Psi_{5}(u)$ & $\alpha_{5}$ & 1 & 1 & $\beta_{5}$ & $u_{1} u_{2}$ \\
\hline
\end{tabular}

Table 4.3: Multi-index: bivariate PCE of $2^{\text {nd }}$ order.

It is straightforward to see a close similarity between the PCE and the HDMR proposed by Sobol. In fact, the output functions can be seen as the sum of higher dimensional terms defined according the multi-index $\alpha_{k}$. In addition, Sobol's condition for the uniqueness of the decomposition requires expressing the output function as the sum of a constant term plus a higher dimensional, zeromean function, a condition that is satisfied thanks to the orthogonality of the polynomials.

Continuing the previous example on a bivariate random variable, Sobol's HDMR considers the terms of increasing dimensionality:

$$
Y(X)=f\left(X_{1}, X_{2}\right)=f_{0}+f_{1}\left(X_{1}\right)+f_{2}\left(X_{2}\right)+f_{12}\left(X_{1}, X_{2}\right),
$$

while looking at the PCE, according to Table 4.3, the following expansion is obtained:

$$
Y(X) \simeq \beta_{0}+\beta_{1} u_{1}+\beta_{2} u_{2}+\beta_{3} u_{1}^{2}+\beta_{4} u_{2}^{2}+\beta_{5} u_{1} u_{2}
$$

Comparing Eq. (4.21) and Eq. (4.22), it is possible to identify the equivalence between the two formulations, which allows an easier calculation of the partial variances and later of the sensitivity indices. 


\begin{tabular}{llll}
\hline Sobol's HDMR & PCE functions & Variance & Variance PCE \\
\hline$f_{0}$ & $\beta_{0}$ & $V\left(f_{0}\right)$ & $\beta_{0}^{2}$ \\
$f_{1}$ & $\beta_{1} u_{1}+\beta_{3} u_{1}^{2}$ & $V\left(f_{1}\right)$ & $\beta_{1}^{2}+\beta_{3}^{2}$ \\
$f_{2}$ & $\beta_{2} u_{2}+\beta_{4} u_{2}^{2}$ & $V\left(f_{2}\right)$ & $\beta_{2}^{2}+\beta_{4}^{2}$ \\
$f_{3}$ & $\beta_{5} u_{1} u_{2}$ & $V\left(f_{3}\right)$ & $\beta_{5}^{2}$ \\
\hline
\end{tabular}

Table 4.4: Equivalence between Sobol's HDMR and PCE.

In fact, the total variance, defined by Eq. (4.20) can be decomposed as:

$$
\begin{aligned}
V(Y) & =V\left(f_{0}\right)+V\left(f_{1}\right)+V\left(f_{2}\right)+V\left(f_{12}\right) \\
& =\beta_{0}^{2}+\left(\beta_{1}^{2}+\beta_{3}^{2}\right)+\left(\beta_{2}^{2}+\beta_{4}^{2}\right)+\beta_{5}^{2},
\end{aligned}
$$

and Sobol's indices can be obtained by the ratio between the partial variances and the total one. For example, the first and total effects with respect the first input variable $X_{1}$ are:

$$
\begin{gathered}
S_{1}^{I}=\frac{V\left(f_{1}\right)}{V(Y)} \approx \frac{\beta_{1}^{2}+\beta_{3}^{2}}{\sum_{m=1}^{p} \beta_{m}^{2}}, \\
S_{1}^{T}=\frac{V\left(f_{1}\right)+V\left(f_{12}\right)}{V(Y)} \approx \frac{\beta_{1}^{2}+\beta_{3}^{2}+\beta_{5}^{2}}{\sum_{m=1}^{p} \beta_{m}^{2}} .
\end{gathered}
$$

Hence the calculation of the PCE coefficients provides a straightforward and computational inexpensive way to estimate the mean, the variance and Sobol's sensitivity indices. It is worth highlighting that Sobol's HDMR is an exact decomposition, while the polynomial expansion would be exact only if the summation were extended to infinite terms. In practice, the PCE is tr uncated to an order that in most applications does not exceed the third degree.

\subsection{A380 RAM Air Inlet free-play nonlinear vibra- tions}

In this section the UQ is considered in the context of an industrial case of study: A380 RAM Air Inlet nonlinear vibrations.

A RAM refers, in this context, to any moving part of a machine that exerts a force onto another one, by pushing or applying pressure on the latter. The RAM Air Inlet(RAI) and the RAM Air Outlet(RAO) openings provide a forced ventilation system to control the air temperature around the Air Generation Unit (AGU). This system supplies and maintains the air in the pressurised fuselage 
compartments at the correct pressure, temperature, and freshness for passenger comfort and equipment cooling. Also, it provides air for ventilation in the unpressurized fuselage bays. Figure 4.2 shows, on the left, the Airbus A380 RAI air intake and two RAO outtakes. Air flow enters through the RAI intake, wets the forward and progressively the reward flaps, enables the AGU heat exchange by forced ventilation, and then comes out through the RAO outtakes.

This study focuses on the RAI system, shown in Figure 4.3: The RAI forward (FWD) and rearward (RWD) flaps are two moving panels whose relative position defines the height of the RAI section and then the amount of air required for heat exchange. In Figure 4.3 two extreme positions are shown: the maximum opening (RAI 100\%) and the minimum one (RAI 0\%). An actuator, through a kinematic chain, shown in Figure 4.4, adjusts the RAI section height and consequently the flaps' relative position. 3D details of the kinematic chain can be seen in Figure 4.5: the actuator drives the shaft, in green, and through two flap links, moves two rods (gray color) which regulate the height by acting on the RWD flap.
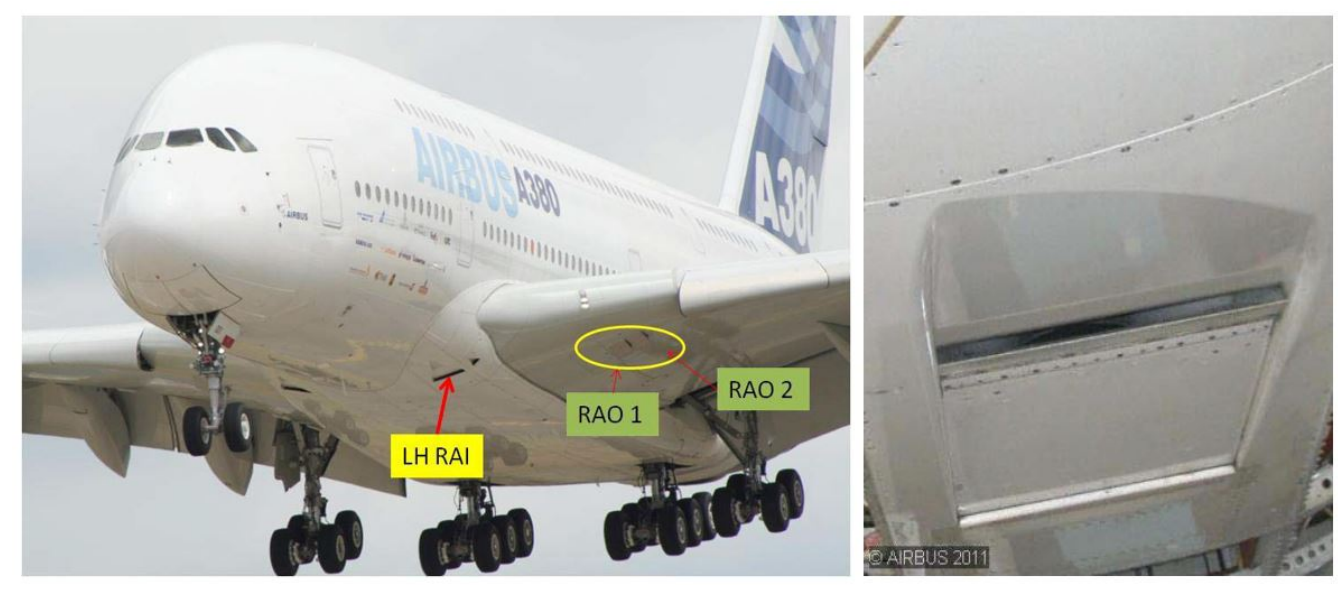

Figure 4.2: A380 - RAI System and Air Intake detail (source|AIRBUS OPERATIONS).

During a flight, the two flaps are subjected to an unsteady pressure field which induces vibrations in the RAI system. Additionally, due to tolerances and wear of the bearings, the connections between the flap links and the rods present free-plays, which make the dynamic problem and hence the rods' reaction forces, nonlinear. Free-play behavior is shown in Figure 4.10 by a nonlinear forcedisplacement curve: the stiffness is zero when the dynamic relative displacements of the interfaces is within a certain tolerance $\delta$ and assumes a value given by 

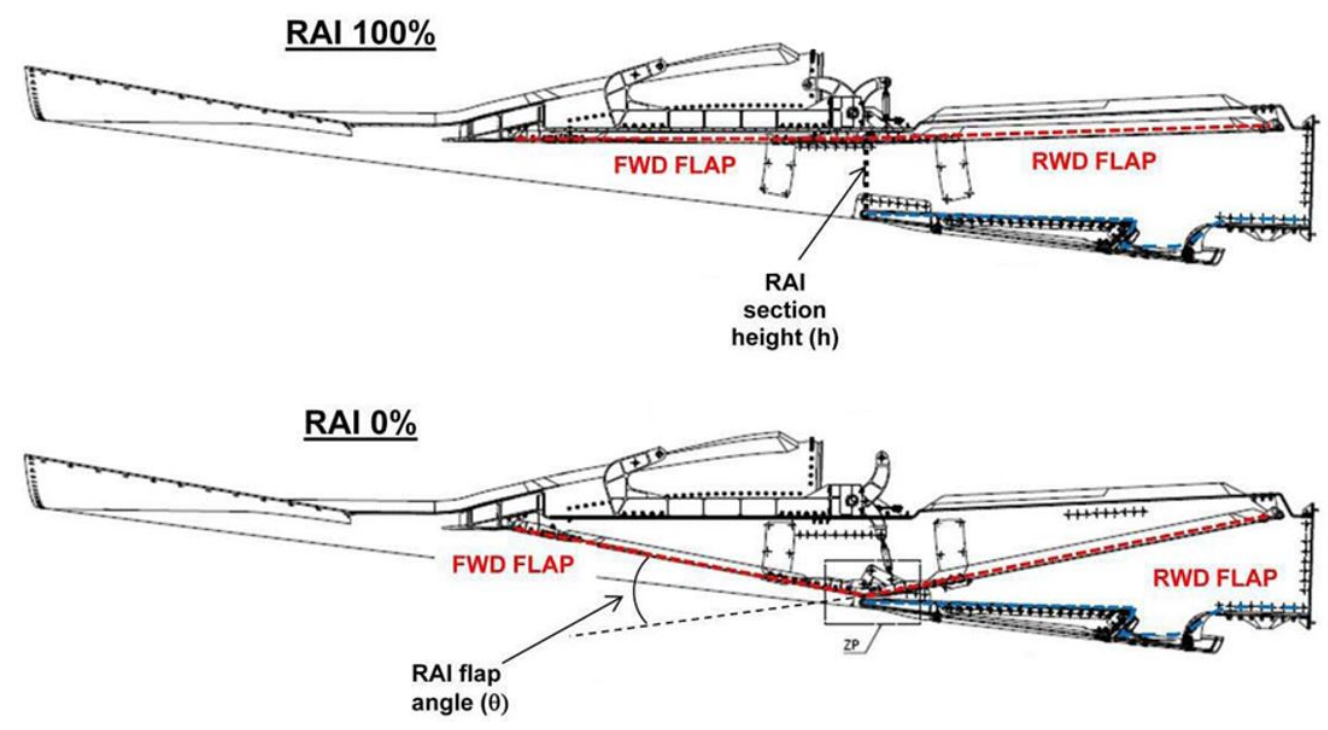

posizione

Figure 4.3: A380 - RAI System Flaps (source|AIRBUS OPERATIONS).

Eq. (4.6) when they are out of this range.

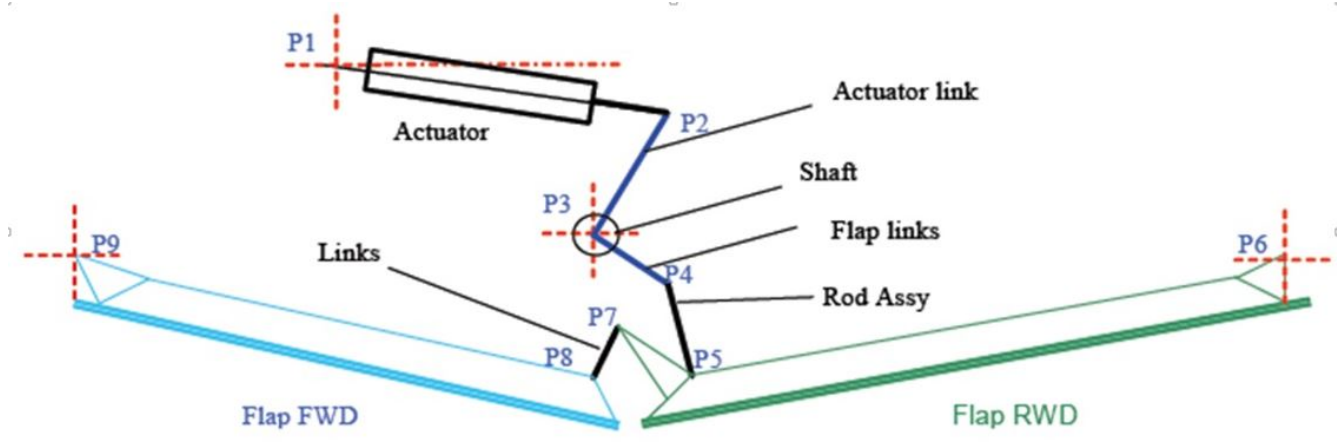

Figure 4.4: Kinematic scheme of flaps control system (source|AIRBUS OPERATIONS).

Large flight test campaigns have facilitated the identification of the worst dynamic load conditions, both in terms of relative flaps' position and unsteady pressure distributions. In all cases, a reliable prediction should take into account the following aspects:

- the free-plays interfaces between the flap links and the rods make the response of the system strongly nonlinear; 


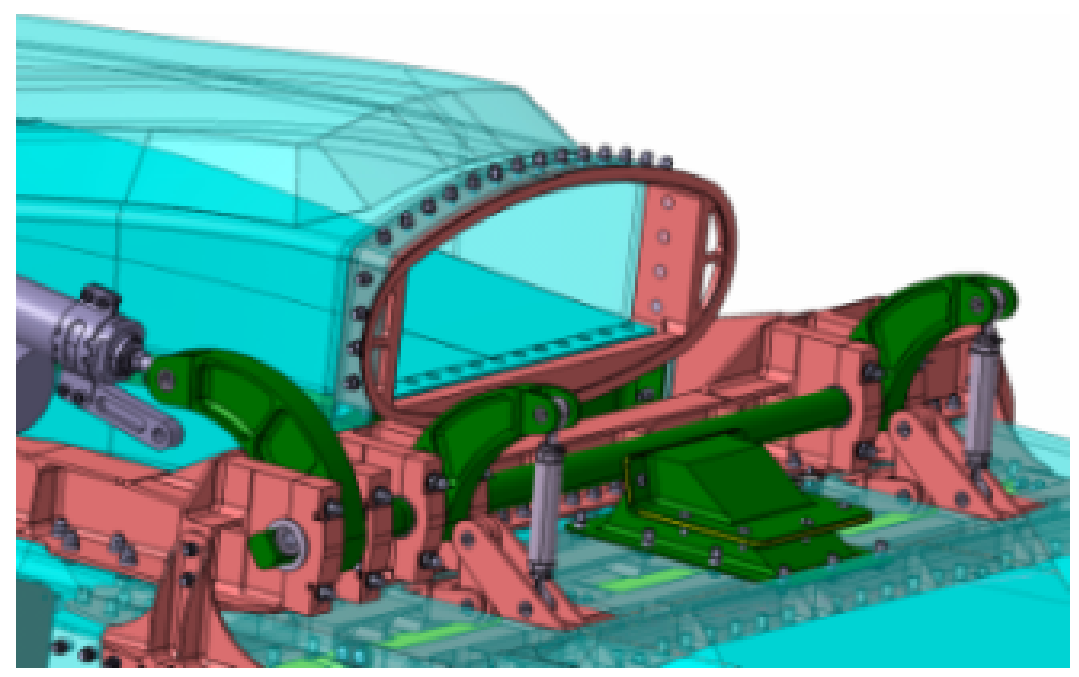

Figure 4.5: 3D design of the flaps control system (source|AIRBUS OPERATIONS).

- the uncertainties regarding both gaps' size $\delta$ and stiffness of the free-plays;

- the uncertainties about the magnitude of the resultant forces on the flaps and their dominant frequency.

\subsubsection{Computational approach}

Based on the previous considerations, a computational approach is proposed next to estimate the stochastic dynamic response of connected structures with uncertain nonlinear interfaces. The study relies on the NL SDMM presented in Section 4.2 to reduce the computational cost associated with each simulation and on Sobol's LDD to sample efficiently the deterministic model. Then, GSA is used to post-process the results and identify the most influential variables of the problem.

The modal matrix is obtained in NASTRAN ${ }^{\circledR}$ from the FE Model showed in Figure 4.6, with the rods disconnected from their arms, or equivalently, by setting the stiffness of the junctions to zero.

First, let us consider applying a set of linear modifications on the model in order to check if the SDMM is able to update the dynamic response in case of a disruptive change of the stiffness at the interfaces, which change from $K_{0}=0$ to $K_{1}=1.5 e^{4}[\mathrm{~N} / \mathrm{mm}]$. The displacements at the interfaces have been evaluated in NASTRAN ${ }^{\circledR}$ considering two models having respectively connected, i.e. $K_{1}$, 


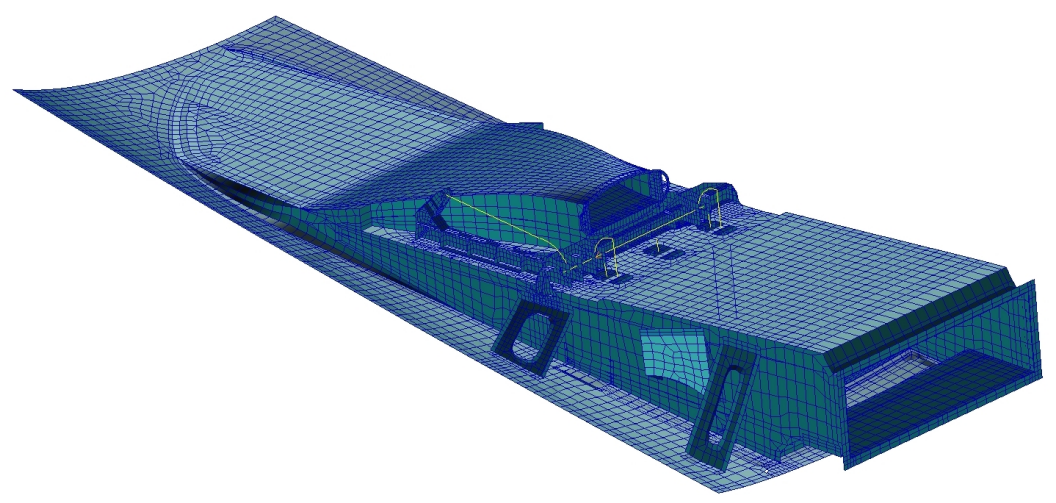

Figure 4.6: RAI FE Model

and disconnected, i.e. $K_{0}$, interfaces. Additionally, by means of the SDMM, the results of the model with connected interfaces have been obtained by updating the disconnected generalized stiffness matrix with an increment of the spring stiffness from $K_{0}$ to $K_{1}$ (see Eq. (4.11)). The comparison, shown in Figure 4.7, in both time and frequency domain, validates the capability of the SDMM to account for a disruptive change of local stiffness.

When rod interfaces are affected by free-plays the system response is no longer linear because the junctions' stiffness depends on the relative displacements of the lumped elements. In particular, Figure 4.8 shows that until the relative displacements are within the interval $[-0.5 ; 0.5][\mathrm{mm}]$, which corresponds to a nominal gap of $1[\mathrm{~mm}]$, the interface stiffness and the rod's reaction force are zero. In contrast, when the gap is saturated, and the absolute value of the relative displacement is greater than $0.5 \mathrm{~mm}$, the interface stiffness has an equivalent value given by Eq. (4.6). Consequently, the nonlinear force is obtained as the product of the equivalent stiffness and the relative displacement. Figure 4.9 compares the rod's reaction force at nominal conditions defined in Table 4.5.

Finally, let us consider interfaces whose behavior is nonlinear and uncertain. The uncertain free-play force-displacement curve is shown in Figure 4.10. Independently for each of the two rods, the size of the gap and stiffness are considered as random variable with prescribed normal distributions according to the values in Table 4.5. Additionally, uniform probability distributions are prescribed on the magnitude and frequency of the force exciting the RWD Flap. When uncertainties are considered, the system's response, and in particular the rods' reactions are uncertain as well. The root mean square (RMS) of the nonlinear rods' reac- 

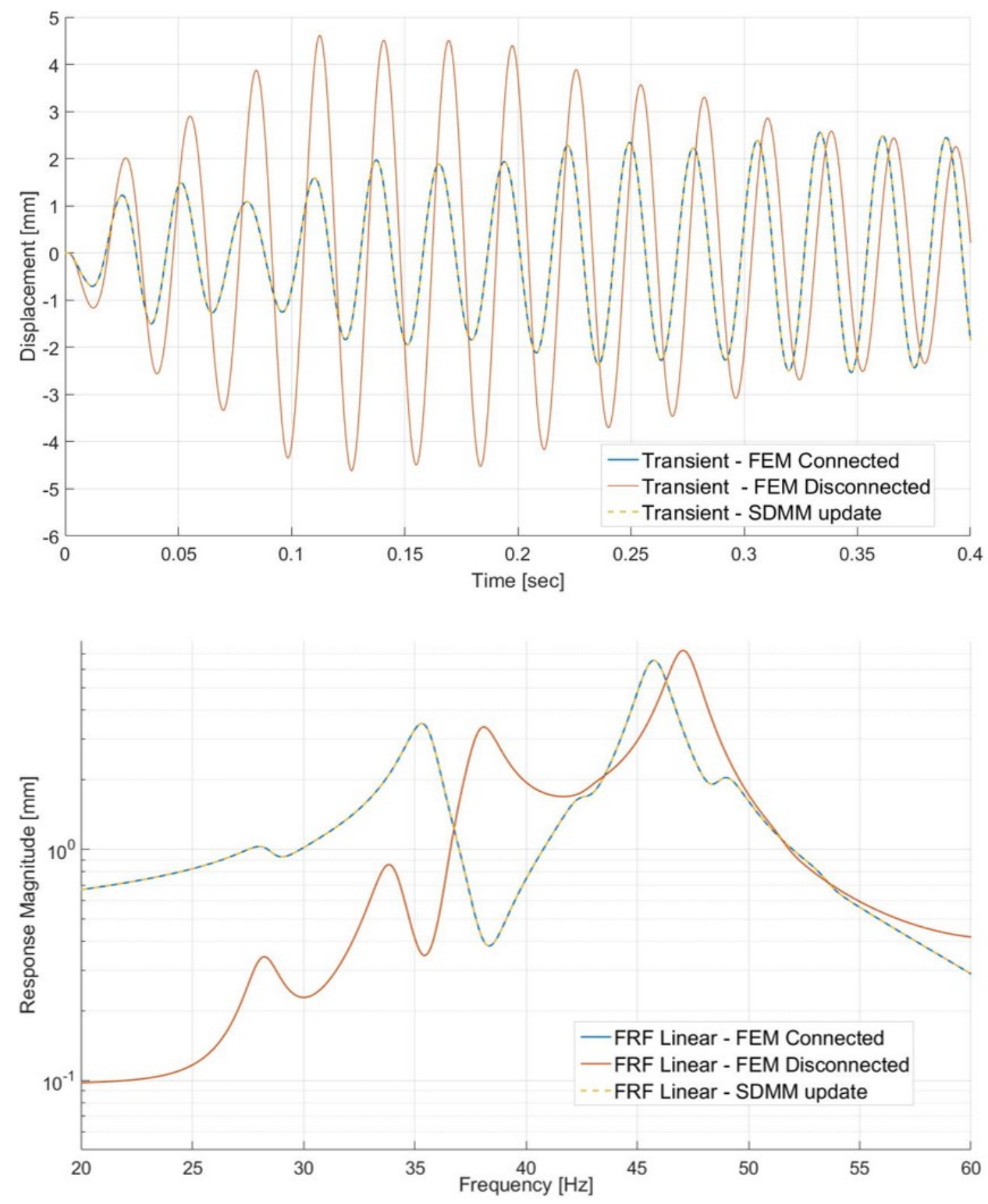

Figure 4.7: SDMM updating method vs. NASTRAN: in this Figure both time and frequency domain response of the system are compared when the springs at interfaces between the rods and the arms are desconnected (red line) and connected (blue line). Both solutions are obtained in NASTRAN. Additionally, the response with connected springs is obtained through updating the disconnected response by means of the SDMM. The obtained response (orange line) overlap the NASTRAN's solution. Hence, the SDMM is able to consider disruptive change of local stiffnesses in both domains. 
tion forces, over a time period of 10 [s], i.e., $F 1_{r m s}$ and $F 2_{r m s}$, are the selected stochastic QoIs. The deterministic model is sampled according a Sobol's LDD of size $N_{\text {run }}=400$, which could be already a very expensive computational cost considering the time required by the model due to its nonlinearities. This initial cost is considerably reduced by the NL SDMM discussed in Section 4.2.

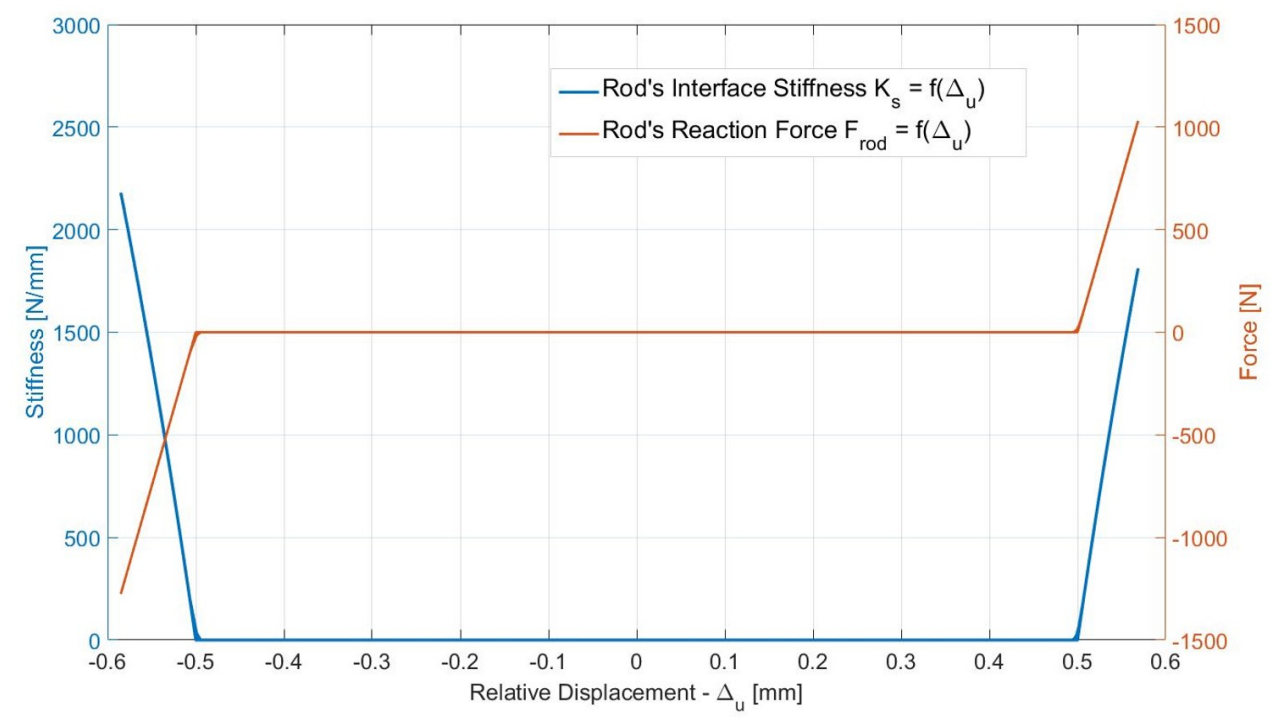

Figure 4.8: Nonlinear stiffness and reaction force at nominal conditions as defined in table 4.5 .

\begin{tabular}{llccc}
\hline Random Variable $\mathbf{x}$ & Distribution & nominal & lower bound & upper bound \\
\hline Force $[\mathrm{N}]$ & Uniform & 500 & 350 & 650 \\
Frequency $[\mathrm{Hz}]$ & Uniform & 35 & 29.5 & 40.5 \\
\hline Random Variable $\mathbf{x}$ & Distribution & mean value & standard deviation & \\
\hline$K_{1}[\mathrm{~N} / \mathrm{mm}]$ & Normal & $1.5 e^{4}$ & $1.5 e^{3}$ & \\
$K_{2}[\mathrm{~N} / \mathrm{mm}]$ & Normal & $1.5 e^{4}$ & $1.5 e^{3}$ & \\
Gap $_{1}[\mathrm{~mm}]$ & Normal & 1.0 & 0.3 & \\
Gap $_{2}[\mathrm{~mm}]$ & Normal & 1.0 & 0.3 & \\
\hline
\end{tabular}

Table 4.5: Statistical distributions of the random variables.

The least square method is used to find the approximation's coefficients, 84 according to Eq. (4.15), of a $3^{\text {rd }}$ order PCE over a sample of $N_{s}=250$ training points. The remaining $N_{e v}=N_{\text {run }}-N_{s}=150$ are reserved exclusively for the 


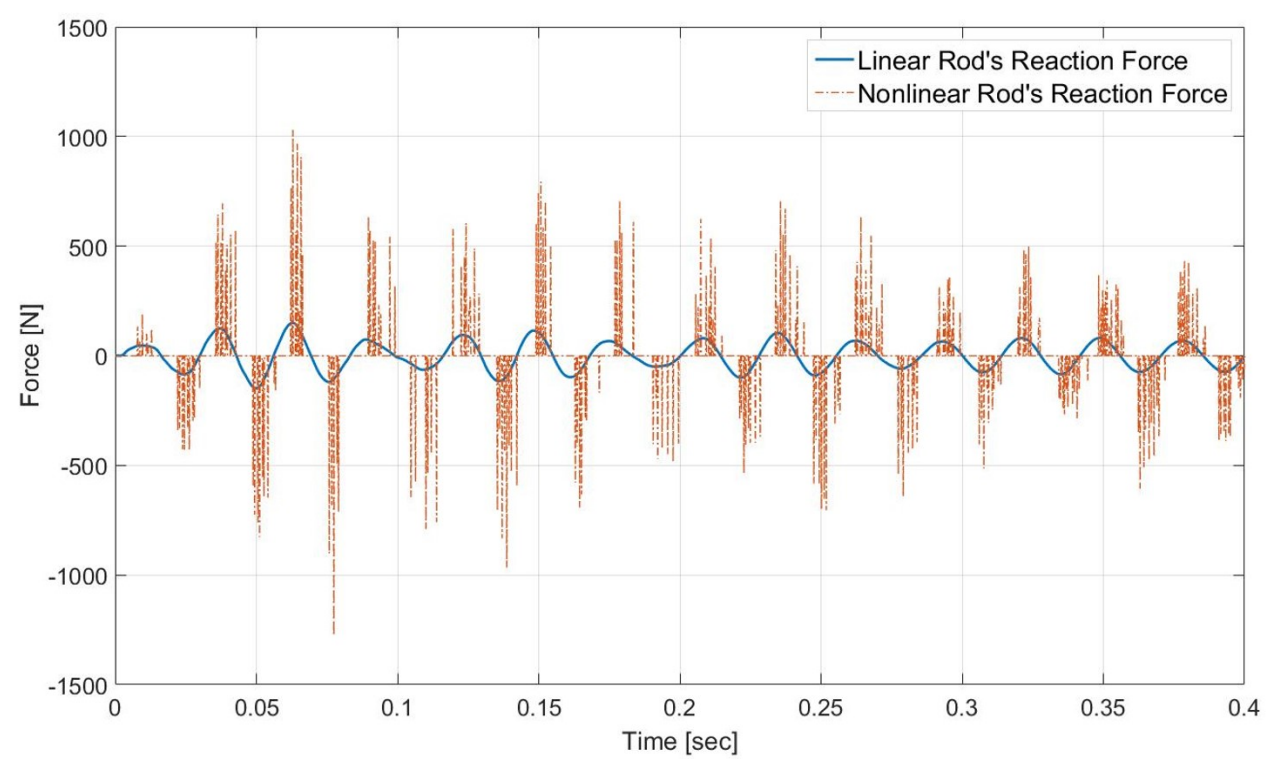

Figure 4.9: Comparison between linear and nonlinear rod's reaction force at nominal conditions as defined in table 4.5.

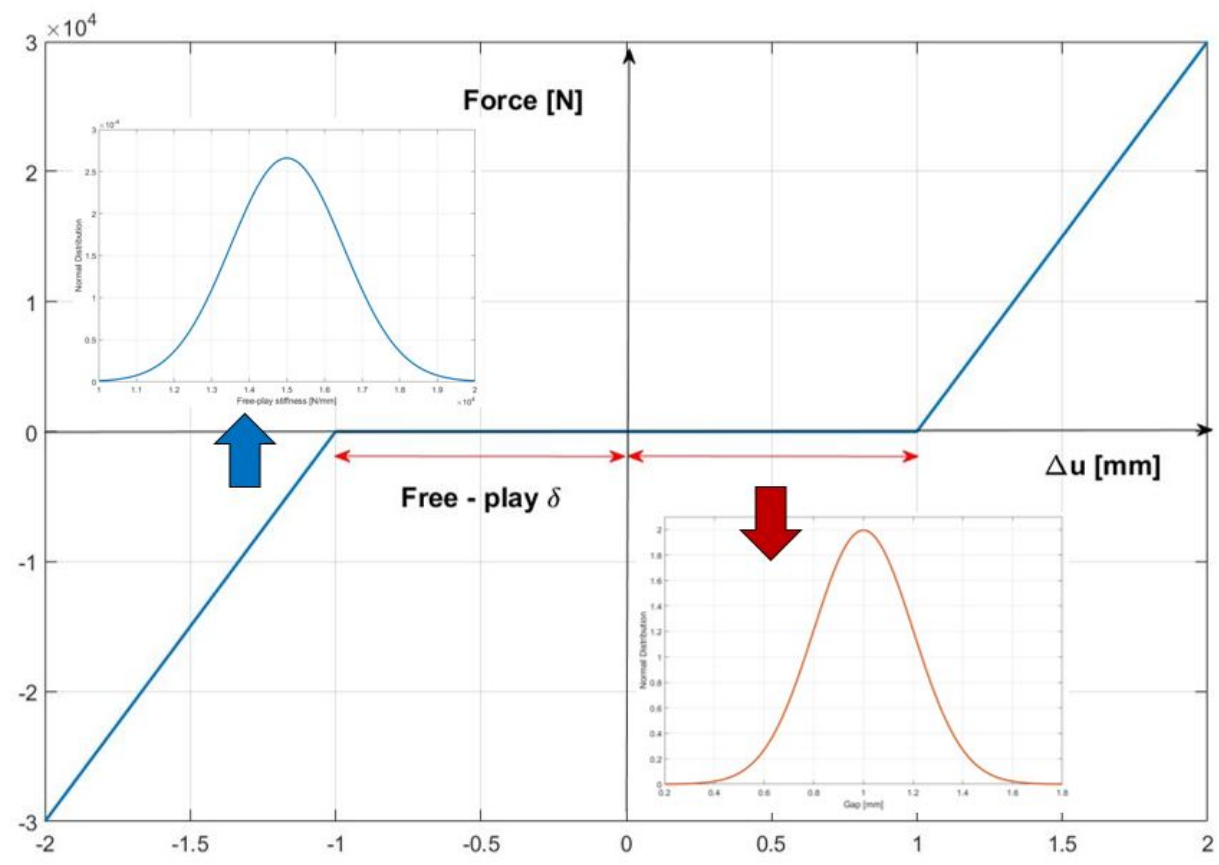

Figure 4.10: Nonlinear interfaces behavior: uncertain free-play stiffness. 
validation of the PCE surrogate and the error on this unseen set of data is:

$$
E r r_{e v}=\frac{\left\|Y_{e v}-\Psi_{e v} \beta\right\|}{N_{e v}\left\|\Psi_{e v} \beta\right\|}
$$

Legendre and Hermite polynomials are respectively employed for the uniform and normal probability distributions according to Table 4.5. The independence of the random variables preserves the orthogonality of the multivariate PCE when different types of polynomials are used. The approximation error is on the of order $10^{-3}$.

Usually, the PCE shows worse performance in case of strong nonlinearities, and increasing the degree of the polynomials does not always guarantee an improvement because of over-fitting: the meta-model trusts the data on the training set too much and performs poorly over unknown sets.

\subsubsection{Results}

Table 4.6 shows the mean, the variance, the error on the evaluation set, and Sobol's sensitivity indices. The frequency is the stochastic parameter which affects the outputs variance the most. This is in agreement with the fact that both intact and disconnect structures present amplification peaks in the prescribed range of variability (Figure 4.7). The nonlinear interfaces behavior is shown in Figure 4.10: the uncertainty on the size of the gaps has a much more relevant effect on the variance of the reaction loads than the uncertainty on the stiffness. According to the results in Table 4.6, there exist differences between the first and total order effects, meaning that the interaction among parameters is significant. This is particularly true for the total indices regarding the gap parameters. It makes sense because some interactions are expected, at least between the applied force and the gap. Then, for example, the $S T_{5}=S_{5}+S_{15}+S_{25}+S_{35}+S_{45}+S_{65}$ is expected to have, at least, the term $S_{15}$ different from zero.

The sensitivity results of the stochastic QoIs $F 1_{r m s}$ are summarized in the pie charts of Figure 4.11. It is worth noting that when the interactions among parameters are significant, the sum of the first order indices is less than one, and the pie chart does not cover the $100 \%$ of the total output variance. In contrast, the sum of the total effects can be greater than one, because for example the factor $S_{15}$ contributes to both $S T_{5}$ and $S T_{1}$, hence the percentages in the pie chart of total effects are redistributed according to the value of their sum. 


\begin{tabular}{cccc}
\hline Parameter & Results & $F 1_{\text {rms }}$ & $F 2_{r m s}$ \\
\hline & $\begin{array}{c}\text { Mean(Y) } \\
\text { V(Y) }\end{array}$ & 140.10 & 207.49 \\
& error on $N_{\text {eval }}$ & 0.0015 & 0.0024 \\
\hline Force & $S_{1}$ & 0.14 & 0.17 \\
Frequency & $S_{2}$ & 0.43 & 0.30 \\
$K_{1}$ & $S_{3}$ & 0.00 & 0.01 \\
$K_{2}$ & $S_{4}$ & 0.00 & 0.01 \\
Gap $_{1}$ & $S_{5}$ & 0.12 & 0.17 \\
Gap $_{2}$ & $S_{6}$ & 0.15 & 0.06 \\
\hline Force & $S T_{1}$ & 0.21 & 0.28 \\
Frequency & $S T_{2}$ & 0.48 & 0.38 \\
$K_{1}$ & $S T_{3}$ & 0.06 & 0.10 \\
$K_{2}$ & $S T_{4}$ & 0.06 & 0.09 \\
Gap $_{1}$ & $S T_{5}$ & 0.20 & 0.27 \\
Gap $_{2}$ & $S T_{6}$ & 0.22 & 0.21 \\
\hline
\end{tabular}

Table 4.6: RAI vibrations - statistical moments and Sobol's Indices.
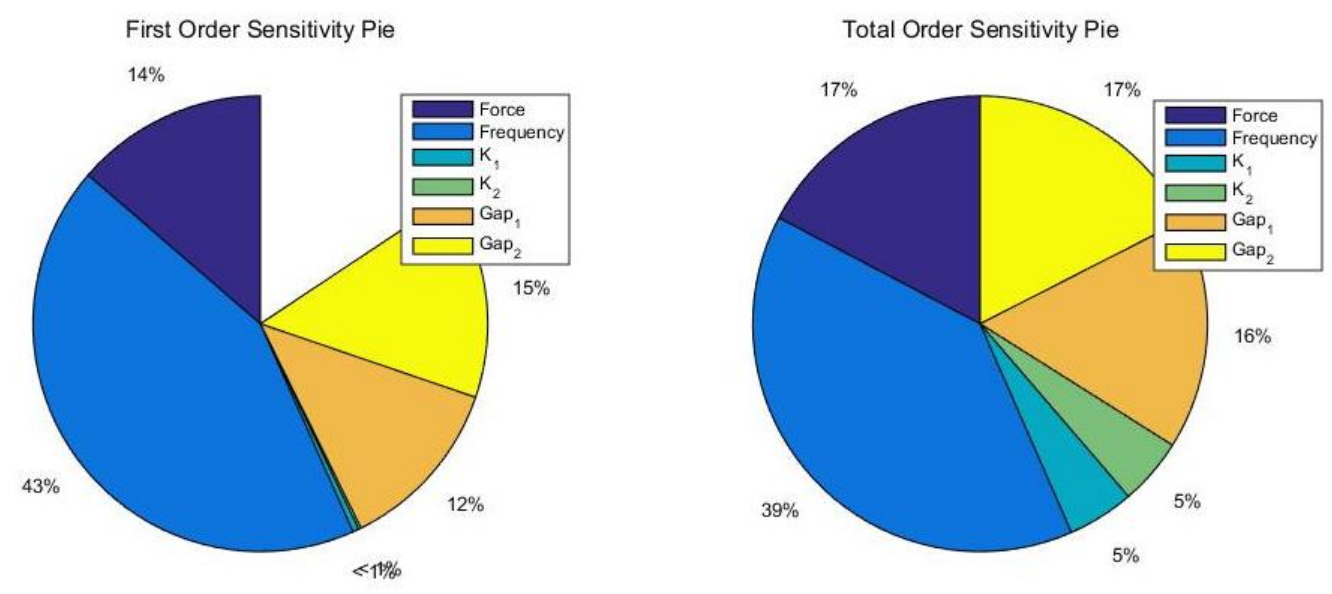

Figure 4.11: Reaction force $F 1_{r m s}$ - Pie charts of first and total indices. The output's variance cannot be explained completely by the first order effects because interactions among inputs are relevant. Particularly, seeing at the total effects, the stiffness $K_{1}$ and $K_{2}$ acquire more relevance. In any case the uncertainty of the response is dominated by the frequency of excitation first, and then by the magnitude of the applied force and by the size of the gaps of free-plays. 


\subsection{Conclusions and future work}

This chapter presents a pragmatic approach for estimating the stochastic dynamic response of assembled structures whose interfaces are nonlinear and uncertain. Non-intrusive, or sample-based, UQ has received more and more attention in the engineering community during the last decade and is a very wide and active field of research. The novelty of this thesis is in incorporating in the UQ process the NL SDMM, which is presented as a reliable and efficient method to avoid the computational burden due to nonlinearities when they can be considered localized and then modeled through lumped elements.

The main issue regarding SDMM, in both linear and nonlinear analyses, is whether the baseline modal base, obtained by using the FE Model, can be updated correctly when the modified local impedance matrix is introduced, or not. The method is more sensitive to the magnitude of the local increments (stiffness, damping, mass) than to the linearity of the modifications. Hence, I consider that free-play behavior is particularly interesting: disruptive changes of local stiffness are considered. A numerical implementation of the SDMM takes care of a reliable modal truncation and eventually to enrich the modal base with specific residual vectors. These are the static vectors of the dofs involved into the modification and can be calculated by applying a unit force on them.

Focus is also put on the similitude between the Sobol's HDMR and the PCE, which allows to obtain the sensitivity indices directly from the coefficients of the expansion. Some theoretical aspects of the key items, i.e., NL SDMM , PCE and GSA, are presented.

The feasibility of the proposed approach is tested on an industrial application. An aircraft component whose dynamic behavior is affected by uncertain free-play junctions is considered and its nonlinear time-domain response is evaluated when it is loaded by a stochastic force. In particular, the root mean square values of the reactions forces at interfaces are selected as QoIs. GSA is proposed as a reliable tool to post-process the outcomes, quantify the effect of each uncertain variable on the QoIs, and select the most relevant ones. The results are discussed looking at their physical coherence and some perspective on the use of PCE in the case of nonlinear problems is given.

The efficiency and predictiveness of meta-models for nonlinear models is an open field of research and further investigations will compare the PCE with other surrogates, such as those based on RBFs. 


\section{Chapter 5}

\section{Summary and conclusions}

Simulations are increasingly being used in supporting or even replacing physical testing in several major industries from civil structural analysis and design to the automotive, aircraft, and naval industries. Two critical aspects of simulationbased analysis and design are the rigorous quantification of uncertainty and the ability to rapidly and accurately assess reliability. Also, sensitivity analysis is a crucial step in the model building and result communication process. Through sensitivity analysis, essential insights on model behavior are gained, on its structure, and on its response to changes in the model variables.

In particular, non-intrusive methods are now established in the engineering community as a pragmatic approach for the UQ and GSA of complex models. The principles behind the variance decomposition and the Sobol's indices are reviewed in chapter 2. In light of an experimental campaign carried out in the laboratory of the Mechanical Engineering department of the UPM, the formulation of Sobol's sensitivities has been reviewed and reformulated. In this experiment, the natural modes of vibrations of a beam with three random masses, whose existence is considered as a binomial variable and hence described by a Bernoulli distribution, are considered. The possible combination of the FFD allow an explicit polynomial regression, then used to calculate analytically Sobol's indices. In literature seldom experimental set-up are employed in the field of UQ and GSA. In particular I am successful in studying an unexplored link, to the best author's knowledge, between the sensitivity indices and the coefficient of determination in regression. Finally, Saltelli's re-sampling method for estimating the Sobol's indices is analyzed in terms of convergence vs. the needed sample size. Even in case of categorical variables, the needed sample size is much higher than the eight simulation points used in the first two approaches.

When a model is computationally expensive or only a set of inputs and the 
corresponding outputs are available, UQ and GSA analyses can only be completed by employing surrogates that replace the original models and are considerably less expensive. The development of meta-models has been a dominant area of research over the last decade. In chapter 3, the construction of accurate and predictive meta-models for their use in both UQ and GSA, and their application to complex problems in non-linear mechanics is presented. In particular, metamodels based on radial functions are examined and enhanced with anisotropic metrics for improved predictiveness and cost effectiveness. Anisotropic kernels are used for obtaining improved performance in particular when a limited set of model evaluations is available. Further, a detailed methodology is described that can be used to systematically construct quasi-optimal meta-models, where a novel link between anisotropic metrics and Sobol's sensitivities is explored and quantified using GSA. In fact what Forrester - in the context of Kriging method - refers to as qualitative connection between relevant variables and active dimensions is revisited and quantified.

As an example of the usefulness of well-designed meta-models, an analytical function, the well-known Sobol's $G$-function, and two mechanical problems are studied by means of surrogates. The sensitivities calculated with my meta-models for the material model of Johnson and Cook related to Taylor's anvil impact test should be particularly interesting for the mechanical engineering community. The identification of the most relevant material parameters can help to improve the experimental set-up for high-velocity impact tests and help in steering experimental campaigns for parameter fitting. Also, a study about the interaction between the impact velocity and the material parameters is presented. This thesis provides the first UQ and GSA analysis which can help the parameter fitting for constitutive models, and guide the formulation of effective meta-models for other similar complex experimental setups.

Finally, chapter 4 considers an application to a real aeronautical structure. The novelty of this thesis is in the incorporation in the UQ process of the NL SDMM, which is presented as a reliable and efficient method to avoid the computational burden due to nonlinearities when they can be considered localized and then modeled through lumped elements. The surrogate in this exercise is obtained by use of multidimensional orthogonal polynomials, i.e., PCE, because focus is put on the similitude between the Sobol's HDMR and the PCE, which allows to obtain the sensitivity indices directly from the coefficients of the expansion. Combining ROM with meta-models is shown as a suitable and pragmatic approach and the methodology presented in this thesis can be extended to all 
those computational models where the uncertainties can be localized at lumped parameters of the FE model.

\subsection{Main results of the thesis}

This thesis is motivated by the advantages that derive, at the industrial level, from the adoption of an effective UQ/GSA framework: a one-time process of product development with increases reliability and durability and the opportunity to provide more credible and realistic simulations. The required additional efforts, essentially an investment in people skills and some extra time in the design and engineering phases of the project, have a clear return of investment in the midand long-term. For the reasons outlined, large companies such as AIRBUS are investing large amounts of funds in the development of rigorous, yet practical, UQ/GSA frameworks that are tightly coupled with their simulation workflows.

Evidently, the creation of well-design mata-models is instrumental to complete UQ and GSA processes. It can happen that the complexity of the simulations puts doubts on surrogate's reliability in particular when they are used as black-box tools. A quasi-optimal meta-model generation integrated in both processes, as an opportunity for improved performance, is presented. That moves the balance of the investment on people skills but greatly reduces that related to the schedule of the project, and consequently makes these techniques more suitable to be integrated and considered in the actual schedule of a project.

Let me recap next the main results of this thesis:

- A clear description and an exhaustive literature review about UQ and GSA processes has been provided.

- A perspective on Sobol's sensitivity indices is given in the light of experimental results and a novel link with the coefficient of approximation in regression is provided. Further, Saltelli's reseampling approach is analyzed in terms of convergence vs. the needed sample size.

- A rigorous framework for metamodels evaluation and selection is provided and a novel methodology to systematically construct quasi-optimal metamodels is defined. In particular a link between anisotropic metrics and Sobol's sensitivities is explored and quantified using GSA.

- The presented concepts and methodologies are successfully applied to real industrial cases. The UQ and GSA analysis of the material model of Johnson and Cook related to Taylor's anvil impact test can help the parameter 
fitting for constitutive models, and guide the formulation of effective metamodels for other similar complex experimental setups. The complex study of nonlinear vibrations of an aeronautic component provides useful information for designers and can be used as guide in order to combine ROM with meta-models.

\section{$5.2 \quad$ Future work}

There are some aspects of this thesis that can be used in further investigations:

- Study the use of anisotropic meta-models with compact support. Having sparse Gram matrices can greatly reduce computer storage requirements and the number of floating point operations needed in computation [96, 97] and I consider that by introducing anisotropic metrics the performance of this class of surrogates could be further improved.

- An extensive use of Sobol's indices is done in this thesis. However, their use to support the construction of quasi-optimal meta-models should be further explored. In this thesis the variables are always considered statistically independent. It is worth to review how Sobol's indices definition changes use in presence of dependent variables $[98,99,100]$ and explore their use in this case. In a related topic, applications should be considered where a mix exists of categorical and continuous random variables. This thesis already provides some guidelines because the experimental campaigns employed the random masses were binomial variables. It has been shown that Saltelli' re-sampling approach works properly with categorical variables but the definition of a metamodel able to include both types of random inputs could be challenging.

- Vibrations issues are very common on aeronautic components, particularly when they are subjected to an external unsteady flow. Facing these problems has not an unique solution and often there are at least two aspects to be considered in order to define modifications of the current design or even a re-design. The first one is the identification of those mechanical elements which contribute to the transmission of vibrations the most. For example, in chapter 4 the RAM Air Inlet, the kinematic chain, and in particular the free-plays of some interfaces were singled out as major sources of vibrations. Then, with the GSA it is possible to quantify the effect of all the random 
variables and sort them according to their relevance. The second aspect is to provide means that will reduce the vibration level. For example, as an extension of the industrial application presented in chapter 4 further investigation can study the optimum locations and properties of nonlinear mass-spring-damper systems to mitigate vibrations [101, 102].

\subsection{Scientific publications and conference presenta- tions}

As a result of the work presented in this thesis the following papers and presentations in conferences are listed next:

- 2020 - A Methodology for the Statistical Calibration of Complex Constitutive Material Models: Application to Temperature-Dependent ElastoVisco-Plastic Materials. Published in Materials.[78]

- 2019 - Anisotropic meta-models for computationally expensive simulations in nonlinear mechanics. Published in International Journal for Numerical Methods in Engineering, Wiley Online Library.[103]

- 2019 - A sample-based approach to estimate the dynamic loads of components with nonlinear uncertain interfaces. Published in Aerospace Science and Technology, Elsevier.[14]

- 2018 - Dynamic analysis of assembled aircraft structures considering interfaces with nonlinear behavior. Published in Aerospace Science and Technology, Elsevier.[54]

- 2018 - A pragmatic approach to estimate the dynamic loads of components with nonlinear uncertain interfaces. Presented at 6th conference of Noise and Vibration Emerging Methods, NOVEM.

- 2017 - Dynamic analysis of assembled structures with nonlinear joints. Presented at 58th AIAA/ASCE/AHS/ASC Structures, Structural Dynamics, and Materials Conference, Grapevine, Texas, USA.[53]

- 2017 - Optimization approach for identification of dynamic parameters of localized joints of aircraft assembled structures. Published in Aerospace Science and Technology, Elsevier.[79] 
- 2017 - An improved software for frequency domain analysis of assembled aircraft structures with local nonlinearities. Presented at 17th International Forum on Aeroelasticity and Structural Dynamics, IFASD.

- 2017 - Uncertainty Quantification in the dynamic response of assembled structures. Presented at 17th International Forum on Aeroelasticity and Structural Dynamics, IFASD.

- 2015 - Nonlinear dynamic analysis of assembled aircraft structures with concentrated nonlinearities. Presented at 16th International Forum on Aeroelasticity and Structural Dynamics, IFASD.[52]

- 2013 - Combined optimization and design of experiment procedures in order to estimate Main Landing Gear Door interface loads under unsteady aerodynamic vibrations. Presented at 15th International Forum on Aeroelasticity and Structural Dynamics, IFASD. 


\section{Appendix A \\ Optimal regularization for ill-conditioning}


At the end of section 3.2.2, the GCV has been introduced as a reliable and convenient model selection criteria particularly in the context of regularization. In fact, Golub [62] showed that it can be expressed as continuous non-linear function of $\lambda$. Moreover, as proposed by Orr [63], setting to zero the GCV derivative a non-linear relationship for the re-estimation of $\lambda$ until convergence can be obtained in order to guarantee at least a local minimum. Inspired by Orr's review, I plan now an efficient and computationally inexpensive approach to ensure the global minimum of the GCV, which can be implemented in few lines of code.

The starting point is the GCV expression [62]:

$$
\operatorname{GCV}(\lambda)=\frac{\operatorname{SSE}(\lambda)}{\operatorname{tr}(P(\lambda))^{2}}
$$

where SSE is the sum of squared errors and $P$ is a projection matrix. In the following, starting from the closed form solution for the regression coefficients $\beta$, I will define the projection matrix and its relationship with the SSE. As a second step, making use of the Singular Value Decomposition (SVD) of $H$, which is computed just once, I demonstrate that the non-linear function $\operatorname{GCV}(\lambda)$ can be sampled, without any other important computational effort, against a range of $\lambda$ values. That permits roughly estimating the GCV minimum and its corresponding regularization parameter $\lambda_{0}$. Finally, the derivative of $\operatorname{GCV}(\lambda)$ allows to obtain an explicit non-linear relationship, whose root gives the global optimum in the considered range of search. This nonlinear equation is then solved to get the optimum value for $\lambda$ considering the initial estimate point $\lambda_{0}$.

The approximation problem of Eq.(3.2) is:

$$
Y \simeq H \beta,
$$

and the minimization of the regularized cost function has a solution given by:

$$
\beta=A^{-1} H^{T} Y,
$$

where $A$ is generally called the Hat Matrix and has the expression:

$$
A=H^{T} H+\lambda I .
$$

Note that $H$ has dimensions $N_{s} \times N_{k}$, hence, both $I$ and $A$ have dimension $N_{k} \times N_{k}$. 
The sum of squared errors is:

$$
\mathrm{SSE}=\|Y-H \beta\|^{2}=\left\|\left(I-H A^{-1} H^{T}\right) Y\right\|^{2}=\|Y\|^{2},
$$

where $P=\left(I-H A^{-1} H^{T}\right)$ is the projection matrix. In fact, $P Y$ is the projection of $Y$ orthogonal to the space spanned by the model (i.e. $N_{k}$, which is the column space of $H$ ) and represents the error between the observed outputs and the least squares estimation.

It is worth noting that in absence of regularization and if $H$ does happen to be a square, invertible matrix, then its column space is the whole space (i.e. $N_{k}=N_{s}$ ), it contains $Y$, and $P$ is the null matrix. The least squares solution is no longer an approximation, but rather an interpolation. The model is forced to fit all the output points and consequently, the SSE is zero. In this case the model is fully constrained to fit the training set of outputs and has zero degrees of freedom. In general, if $N_{k}$ centers are used over the $N_{s}<N_{k}$ training outputs, the $N_{k}$ regression coefficients are estimated leaving out $N_{s}-N_{k}$ degrees of freedom. The unbiased estimate of the variance is thus

$$
\sigma^{2}=\frac{\mathrm{SSE}}{N_{s}-N_{k}}
$$

In statistics and machine learning this problem is known as the bias-variance dilemma: the model has to capture accurately the regularities in the training data, but also generalizes well to unseen data. The trace of the projection matrix, $\operatorname{tr}(P)$, defines the effective degree of freedoms. In absence of regularization $\operatorname{tr}(P)=N_{s}-N_{k}$, while in the case of a penalty factor, it is:

$$
\begin{aligned}
\operatorname{tr}(P) & =\operatorname{tr}\left(I_{N_{s}}-H A^{-1} H^{T}\right) \\
& =N_{s}-\operatorname{tr}\left(A^{-1}\left(A-\lambda I_{N_{k}}\right)\right) \\
& =N_{s}-\operatorname{tr}\left(I_{N_{k}}-\lambda A^{-1}\right) \\
& =N_{s}-N_{k}+\lambda \operatorname{tr}\left(A^{-1}\right) .
\end{aligned}
$$

The regularization parameter increases the effective degrees of freedom. In fact, even in the case of a square $H$ matrix, i.e. $N_{s}=N_{k}$, some bias is introduced in the solution of the problem.

Now, the function $\operatorname{GCV}(\lambda)$ is expressed in terms of the SVD of the kernel matrix, $H=U S V^{T}$, where $U, N_{s} \times N_{k}$, and $V, N_{k} \times N_{k}$, are orthogonal matrices and $S$ is the $N_{k} \times N_{k}$ diagonal matrix of the singular values of $H$. 
The projection matrix is then

$$
\begin{aligned}
P & =I-H A^{-1} H^{T} \\
& =I_{N_{s}}-U\left(S^{2}\left(S^{2}+\lambda I_{N_{k}}\right)^{-1}\right) U^{T} \\
& =I_{N_{s}}-U F U^{T},
\end{aligned}
$$

where $F$ is called the filter matrix. It is diagonal and its generic component is

$$
f_{k k}=\frac{s_{k k}^{2}}{s_{k k}^{2}+\lambda}
$$

and the regularized solution becomes

$$
\tilde{Y}=H \beta=H A^{-1} H^{T} Y=U F U^{T} Y .
$$

Therefore, like the standard regression, it computes the coordinates of $Y$ projected in the orthonormal basis $U$, but these coordinates are shrunk by the filter matrix $F$, which is different from identity for any $\lambda>0$. Additionally, the small singular values $s_{k k}$ correspond to directions in the column space of $H$ having small variance, and the $\lambda$ shrinks these directions the most. The regularization protects against the potentially high variance of gradient estimated in short directions.

Since $P=I_{N_{s}}-U F U^{T}$, the trace of $P$ is

$$
\operatorname{tr}(P)=N_{s}-\operatorname{tr}(F)
$$

and finally, it is

$$
\operatorname{GCV}(\lambda)=\frac{\left\|\left(I_{N_{s}}-U F(\lambda) U^{T}\right) Y\right\|^{2}}{\left[N_{s}-\operatorname{tr}(F(\lambda)]^{2}\right.} .
$$

Once the SVD of $H$ is obtained, Eqs. (A.8) and (A.11) provide the means to estimate the GCV model estimator for different values of $\lambda$. In the algorithm, 200 logarithmically distributed $\lambda$ are used to plot the GCV and obtain an initial guess $\lambda_{0}$ for the optimum value of $\lambda$.

In the last step the derivative of the GCV with respect to $\lambda$ is calculated. Setting it to zero, a non-linear explicit relationship is obtained which allows to find the optimal value of the regularization parameter and consequently the minimum of the GCV. Imposing

$$
\frac{\partial \mathrm{GCV}(\lambda)}{\partial \lambda}=0,
$$

the following non-linear equation is obtained: 


$$
\lambda-\frac{\operatorname{SSE}(\lambda) \sum \frac{s_{k}^{2}}{\left(s_{k}^{2}+\lambda\right)^{2}}}{\operatorname{tr}(P(\lambda)) \sum \frac{z_{k}^{2} s_{k}^{2}}{\left(s_{k}^{2}+\lambda\right)^{3}}}=0,
$$

where the $z_{k}$ are the components of the vector $Z=U^{T} Y$. As already mentioned, this nonlinear equation can be solved using as initial estimate the previously found value $\lambda_{0}$. 


\section{Bibliography}

[1] Smith RC. Uncertainty quantification: theory, implementation, and applications, vol. 12. Siam, 2013.

[2] Simpson TW, Poplinski J, Koch PN, Allen JK. Metamodels for computerbased engineering design: survey and recommendations. Engineering with Computers 2001; 17(2):129-150.

[3] Wang GG, Shan S. Review of metamodeling techniques in support of engineering design optimization. Journal of Mechanical Design 2007; 129(4):370-380.

[4] Sudret B. Global sensitivity analysis using polynomial chaos expansions. Reliability Engineering \& System Safety 2008; 93(7):964-979.

[5] Raisee M, Kumar D, Lacor C. A non-intrusive model reduction approach for polynomial chaos expansion using proper orthogonal decomposition. International Journal for Numerical Methods in Engineering 2015; 103(4):293312.

[6] Der Kiureghian A, Ditlevsen O. Aleatory or epistemic? does it matter? Structural Safety 2009; 31(2):105-112.

[7] Press SJ. Applied multivariate analysis: using Bayesian and frequentist methods of inference. Courier Corporation, 2005.

[8] Grandhi RV, Fischer CC. Model-form uncertainty quantification for structural design. Encyclopedia of Earthquake Engineering 2014; :1-17.

[9] Guedri M, Cogan S, Bouhaddi N. Robustness of structural reliability analyses to epistemic uncertainties. Mechanical systems and signal processing 2012; 28:458-469. 
[10] Mullins J, Ling Y, Mahadevan S, Sun L, Strachan A. Separation of aleatory and epistemic uncertainty in probabilistic model validation. Reliability Engineering \& System Safety 2016; 147:49-59.

[11] Rabhi N, Guedri M, Hassis H, Bouhaddi N. Structure dynamic reliability: A hybrid approach and robust meta-models. Mechanical systems and signal processing 2011; 25(7):2313-2323.

[12] Adhikari S. Damping model uncertainty in structural dynamics. Proceedings of the International Conference on Noise and Vibration Engineering (ISM), 2006.

[13] Van Buren KL, Hall TM, Gonzales LM, Hemez FM, Anton SR. A case study to quantify prediction bounds caused by model-form uncertainty of a portal frame. Mechanical Systems and Signal Processing 2015; 50:11-26.

[14] Menga E, Sánchez M, Romero I, Hernández S. A sample-based approach to estimate the dynamic loads of components with nonlinear uncertain interfaces. Aerospace Science and Technology 2019; 87:369-378.

[15] Agresti A. An introduction to categorical data analysis. John Wiley \& Sons, 2018.

[16] Tutz G. Regression for categorical data, vol. 34. Cambridge University Press, 2011.

[17] Swiler LP, Hough PD, Qian P, Xu X, Storlie C, Lee H. Surrogate models for mixed discrete-continuous variables. Constraint Programming and Decision Making. Springer, 2014; 181-202.

[18] Bauman L. New methods of uncertainty quantification for mixed discretecontinuous variable models. Sandia National Laboratories (SNL-CA), Livermore, CA (United States) 2013; .

[19] Halton JH. On the efficiency of certain quasi-random sequences of points in evaluating multi-dimensional integrals. Numerische Mathematik 1960; 2(1):84-90.

[20] Sobol IM. Uniformly distributed sequences with an additional uniform property. USSR Computational Mathematics and Mathematical Physics 1976; 16(5):236-242. 
[21] Saltelli A, Ratto M, Andres T, Campolongo F, Cariboni J, Gatelli D, Saisana M, Tarantola S. Global sensitivity analysis: the primer. John Wiley \& Sons, 2008.

[22] Pianosi F, Beven K, Freer J, Hall JW, Rougier J, Stephenson DB, Wagener T. Sensitivity analysis of environmental models: A systematic review with practical workflow. Environmental Modelling \& Software 2016; 79:214-232.

[23] Sudret B. Meta-models for structural reliability and uncertainty quantification. arXiv preprint arXiv:1203.2062 2012; .

[24] Forrester A, Sobester A, Keane A. Engineering design via surrogate modelling: a practical guide. John Wiley \& Sons, 2008.

[25] Schilders WH, Van der Vorst HA, Rommes J. Model order reduction: theory, research aspects and applications, vol. 13. Springer, 2008.

[26] Chinesta F, Ladeveze P, Cueto E. A short review on model order reduction based on proper generalized decomposition. Archives of Computational Methods in Engineering 2011; 18(4):395.

[27] Blatman G, Sudret B. Sparse polynomial chaos expansions and adaptive stochastic finite elements using a regression approach. Comptes Rendus Mécanique 2008; 336(6):518-523.

[28] Schobi R, Sudret B, Wiart J. Polynomial-chaos-based kriging. International Journal for Uncertainty Quantification 2015; 5(2).

[29] Simpson TW, Mauery TM, Korte JJ, Mistree F. Kriging models for global approximation in simulation-based multidisciplinary design optimization. AIAA journal 2001; 39(12):2233-2241.

[30] Martin JD, Simpson TW. Use of kriging models to approximate deterministic computer models. AIAA journal 2005; 43(4):853-863.

[31] Fasshauer GE. Meshfree Approximation Methods with Matlab, vol. 6. World Scientific Publishing Co Inc, 2007.

[32] Fasshauer G, McCourt M. Kernel-based approximation methods using Matlab, vol. 19. World Scientific Publishing Co Inc, 2015.

[33] Forrester AI, Keane AJ. Recent advances in surrogate-based optimization. Progress in aerospace sciences $2009 ;$ 45(1-3):50-79. 
[34] Bhosekar A, Ierapetritou M. Advances in surrogate based modeling, feasibility analysis, and optimization: A review. Computers 85 Chemical Engineering 2018; 108:250-267.

[35] Baudin M, Martinez J. Introduction to polynomials chaos with nisp 2016.

[36] Augustin F, Gilg A, Paffrath M, Rentrop P, Wever U. Polynomial chaos for the approximation of uncertainties: Chances and limits. European Journal of Applied Mathematics 2008; 19(2):149-190.

[37] J Toal D, Bressloff NW, Keane AJ. Kriging hyperparameter tuning strategies. AIAA journal 2008; 46(5):1240-1252.

[38] Sobol IM. Sensitivity estimates for nonlinear mathematical models. Mathematical modelling and computational experiments 1993; 1(4):407-414.

[39] Sobol IM. Theorems and examples on high dimensional model representation. Reliability Engineering \& System Safety 2003; 79(2):187-193.

[40] Hoeffding W. A class of statistics with asymptotically normal distribution. Breakthroughs in Statistics. Springer, 1992; 308-334.

[41] Homma T, Saltelli A. Importance measures in global sensitivity analysis of nonlinear models. Reliability Engineering 83 System Safety 1996; 52(1):117.

[42] Baudin M, Martinez J. Introduction to sensitivity analysis with nisp. Version 0.52014 ; .

[43] Mandel D. Random sobol'sensitivity analysis and model robustness 2017; .

[44] Cukier R, Schaibly J, Shuler KE. Study of the sensitivity of coupled reaction systems to uncertainties in rate coefficients. iii. analysis of the approximations. The Journal of Chemical Physics 1975; 63(3):1140-1149.

[45] Saltelli A, Tarantola S, Chan KS. A quantitative model-independent method for global sensitivity analysis of model output. Technometrics 1999; 41(1):39-56.

[46] Bang Y, Abdel-Khalik HS, Hite JM. Hybrid reduced order modeling applied to nonlinear models. International Journal for Numerical Methods in Engineering 2012; 91(9):929-949. 
[47] Montgomery DC, Runger GC. Applied statistics and probability for engineers. John Wiley and Sons, 2014.

[48] Berger VW, Zhou Y. Kolmogorov-smirnov test: Overview. Wiley statsref: Statistics reference online 2014; .

[49] Mendez H. Understanding the central limit theorem. 1993; .

[50] Casciola G, Lazzaro D, Montefusco LB, Morigi S. Shape preserving surface reconstruction using locally anisotropic radial basis function interpolants. Computers \& Mathematics with Applications 2006; 51(8):1185-1198.

[51] Casciola G, Montefusco LB, Morigi S. The regularizing properties of anisotropic radial basis functions. Applied Mathematics and Computation 2007; 190(2):1050-1062.

[52] Menga E, Hernández S, Moledo S, López C. Nonlinear dynamic analysis of assembled aircraft structures with concentrated nonlinearities. 16th International Forum on Aeroelasticity and Structural Dynamics, IFASD, 2015.

[53] Hernández S, Menga E, Naveira P, López C, Baldomir A, Cid M, Moledo S, Freire D. Dynamic analysis of assembled structures with nonlinear joints. 58th AIAA/ASCE/AHS/ASC Structures, Structural Dynamics, and Materials Conference, Grapevine, Texas, USA, 2017.

[54] Hernández S, Menga E, Naveira P, Freire D, López C, Montoya MC, Moledo $\mathrm{S}$, Baldomir A. Dynamic analysis of assembled aircraft structures considering interfaces with nonlinear behavior. Aerospace Science and Technology 2018; 77:265-272.

[55] Saltelli A. Making best use of model evaluations to compute sensitivity indices. Computer physics communications 2002; 145(2):280-297.

[56] Saltelli A, Annoni P. How to avoid a perfunctory sensitivity analysis. Environmental Modelling \& Software 2010; 25(12):1508-1517.

[57] Saltelli A, Annoni P, Azzini I, Campolongo F, Ratto M, Tarantola S. Variance based sensitivity analysis of model output. design and estimator for the total sensitivity index. Computer Physics Communications 2010; 181(2):259-270.

[58] Friedman J, Hastie T, Tibshirani R. The elements of statistical learning, vol. 1. Springer series in statistics (New York), 2001. 
[59] Rippa S. An algorithm for selecting a good value for the parameter $\mathrm{c}$ in radial basis function interpolation. Advances in Computational Mathematics 1999; 11(2):193-210.

[60] Hyndman R. Why every statistician should know about cross-validation. URL http://robjhyndman.com/hyndsight/crossvalidation/.

[61] Arlot S, Celisse A. A survey of cross-validation procedures for model selection. Statistics surveys 2010; 4:40-79.

[62] Golub GH, Heath M, Wahba G. Generalized cross-validation as a method for choosing a good ridge parameter. Technometrics 1979; 21(2):215-223.

[63] Orr MJ. Introduction to radial basis function networks. Technical Report, Center for Cognitive Science, University of Edinburgh 1996.

[64] Honerkamp J, Weese J. Tikhonov's regularization method for ill-posed problems. Continuum Mechanics and Thermodynamics 1990; 2(1):17-30.

[65] Golub GH, Hansen PC, O'Leary DP. Tikhonov regularization and total least squares. SIAM Journal on Matrix Analysis and Applications 1999; 21(1):185-194.

[66] Wendland H. Scattered data approximation, Volume 17 of Cambridge Monographs on Applied and Computational Mathematics, vol. 28. Cambridge University Press, Cambridge, 2005.

[67] Surjanovic S, Bingham D. Virtual library of simulation experiments: Test functions and datasets. URL https://www.sfu.ca/ ssurjano/uq.html.

[68] Marrel A, Iooss B, Laurent B, Roustant O. Calculations of sobol indices for the gaussian process metamodel. Reliability Engineering \& System Safety 2009; 94(3):742-751.

[69] Taylor G. The use of flat-ended projectiles for determining dynamic yield stress-I. theoretical considerations. Proceedings of the Royal Society of London. Series A, Mathematical and Physical Sciences 1948; :289-299.

[70] Whiffin A. The use of flat-ended projectiles for determining dynamic yield stress-II. Tests on various metallic materials. Proc. R. Soc. Lond. A 1948; 194(1038):300-322.

[71] Gust W. High impact deformation of metal cylinders at elevated temperatures. Journal of Applied Physics 1982; 53(5):3566-3575. 
[72] Brünig M, Driemeier L. Numerical simulation of Taylor impact tests. International Journal of Plasticity 2007; 23(12):1979-2003.

[73] Banerjee A, Dhar S, Acharyya S, Datta D, Nayak N. Determination of Johnson-Cook material and failure model constants and numerical modelling of Charpy impact test of armour steel. Materials Science and Engineering: A 2015; 640:200-209.

[74] Samantaray D, Mandal S, Bhaduri A. A comparative study on Johnson Cook, modified Zerilli-Armstrong and Arrhenius-type constitutive models to predict elevated temperature flow behaviour in modified $9 \mathrm{Cr}-1 \mathrm{Mo}$ steel. Computational Materials Science 2009; 47(2):568-576.

[75] Johnson GR. A constitutive model and data for metals subjected to large strains, high strain rates and high temperatures. Proceedings of the 7th International Symposium on Ballistics, The Hague, Netherlands, 1983, 1983.

[76] Johnson GR, Cook WH. Fracture characteristics of three metals subjected to various strains, strain rates, temperatures and pressures. Engineering Fracture Mechanics 1985; 21(1):31-48.

[77] Portillo D, del Pozo D, Rodríguez-Galán D, Segurado J, Romero I. Muesli - A Material UnivErSal LIbrary. Advances in Engineering Software 2017; 105:1-8.

[78] de Pablos J, Menga E, Romero I. A methodology for the statistical calibration of complex constitutive material models: Application to temperaturedependent elasto-visco-plastic materials. Materials 2020; 13(1):19.

[79] Hernández S, Menga E, Moledo S, Romera L, Baldomir A, López C, Montoya MC. Optimization approach for identification of dynamic parameters of localized joints of aircraft assembled structures. Aerospace Science and Technology 2017; 69:538-549.

[80] Tol Ş, et al.. Dynamic characterization of bolted joints using frf decoupling and optimization. Mechanical Systems and Signal Processing 2015; 54:124138.

[81] Bograd S, Reuss P, Schmidt A, Gaul L, Mayer M. Modeling the dynamics of mechanical joints. Mechanical Systems and Signal Processing 2011; 25(8):2801-2826. 
[82] Ibrahim R, Pettit C. Uncertainties and dynamic problems of bolted joints and other fasteners. Journal of sound and Vibration 2005; 279(3-5):857936.

[83] Gangadharan S, Nikoladaidis E. Probabilistic system identification of two flexible joint models. AIAA Journal 1991; 29(8):1319-1326.

[84] Walther H, Kmetyk L. Bolted joints: Model uncertainty vs. test variability. Proceedings of the 22nd IMAC, Dearborn, Michigan, (USA), 2004.

[85] Kladitis P, Bright V, Kharoufeh J. Uncertainty in manufacture and assembly of multipe-joint solder self-assembed microelectromechanical systems (mems). Journal of Manufacturing Processes 2004; 6(1):32-50.

[86] Sommerwerk K, Michels B, Lindhorst K, Haupt M, Horst P. Application of efficient surrogate modeling to aeroelastic analyses of an aircraft wing. Aerospace Science and Technology 2016; 55:314-323.

[87] Lu C, Feng YW, Liem RP, Fei CW. Improved kriging with extremum response surface method for structural dynamic reliability and sensitivity analyses. Aerospace Science and Technology 2018; 76:164-175.

[88] Sestieri A. Structural dynamic modification. Sadhana 2000; 25(3):247-259.

[89] Nad' M. Structural dynamic modification of vibrating systems. Applied and Computational Mechanics 2007; 1(1):203-214.

[90] Kalaycioğlu T, Özgüven HN. Nonlinear structural modification and nonlinear coupling. Mechanical Systems and Signal Processing 2014; 46(2):289306.

[91] Kalaycioğlu T, Özgüven HN. Harmonic response of large engineering structures with nonlinear modifications. Proceedings of the 8th international conference on structural dynamics, EURODYN, 2011; 3623-3629.

[92] Wiener N. The homogoeneous chaos. American Journal of Mathematics 1938; 60(4):897-936.

[93] Askey R, Wilson J. Some basic hypergeometric polynomials that generalize jacobi polynomials. Memoirs of the American Mathematical Society 319, AMS, Providence, RI 1985; . 
[94] Xiu D, Karniadakis GE. The wiener-askey polynomial chaos for stochastic differential equations. SIAM Journal of Scientific Computing 2003; 187:137-167.

[95] Hosder S, Walters R, Balch M. Efficient sampling for non-intrusive polynomial chaos applications with multiple uncertain input variables. Proceedings of the 48th AIAA/ASME/ASCE/AHS/ASC Structures, Structural Dynamics, and Materials Conference, No. AIAA-2007-1939, Honolulu, HI, April 23-26 2007; .

[96] Zhang HH, Genton MG, Liu P. Compactly supported radial basis function kernels. Technical Report, North Carolina State University. Dept. of Statistics 2004 .

[97] Bozzini M, Lenarduzzi L, Rossini M, Schaback R. Interpolation with variably scaled kernels. IMA Journal of Numerical Analysis 2015; 35(1):199219.

[98] Kucherenko S, Munoz Zuniga M, Tarantola S, Annoni P. Metamodelling and global sensitivity analysis of models with dependent variables. AIP Conference Proceedings, vol. 1389, American Institute of Physics, 2011; 1913-1916.

[99] Iooss B, Prieur C. Shapley effects for sensitivity analysis with dependent inputs: comparisons with sobol' indices, numerical estimation and applications. arXiv preprint arXiv:1707.01334 2017; .

[100] Chastaing G, Gamboa F, Prieur C, et al.. Generalized hoeffding-sobol decomposition for dependent variables-application to sensitivity analysis. Electronic Journal of Statistics 2012; 6:2420-2448.

[101] Park KS, Koh HM, Hahm D. Integrated optimum design of viscoelastically damped structural systems. Engineering Structures 2004; 26(5):581-591.

[102] Frans R, Arfiadi Y. Designing optimum locations and properties of mtmd systems. Procedia Engineering 2015; 125:892-898.

[103] Menga E, Sánchez MJ, Romero I. Anisotropic meta-models for computationally expensive simulations in nonlinear mechanics. International Journal for Numerical Methods in Engineering 2020; 121(5):904-924. 